\title{
DOUBLES MÉLANGES DES POLYLOGARITHMES MULTIPLES AUX RACINES DE L'UNITÉ
}

\author{
par Georges RACINET
}

\begin{abstract}
RÉSUMÉ
Les valeurs des fonctions zêta multiples aux entiers strictement positifs fournissent une solution au système d'équations des associateurs de Drinfel'd, aux nombreuses applications en algèbre quantique. Vues comme intégrales itérées, ce sont les périodes du groupoïde fondamental motivique de $\mathbf{P}^{1} \backslash\{0,1, \infty\}$, d'où un système fondamental de relations algébriques, qui implique celui des associateurs mais n’est pas explicite.

On étudie ici la combinatoire d'un autre système de relations, les doubles mélanges, qui provient de manipulations élémentaires de séries et d'intégrales. On montre qu'il partage une propriété importante avec les associateurs et les relations "motiviques», est conséquence de ces dernières et définit une algèbre de polynômes sur $\mathbf{Q}$ (théorème d'Écalle). On obtient ces résultats pour les nombres plus généraux que sont les polylogarithmes multiples aux racines de l'unité de Goncharov.
\end{abstract}

\section{ABSTRACT}

The values at positive integers of the polyzeta functions are solutions of the polynomial equations arising from Drinfeld's associators, which have numerous applications in quantum algebra. As iterated integrals they are periods of the motivic fundamental groupoid of $\mathbf{P}^{1} \backslash\{0,1, \infty\}$. From this arises a fundamental, yet no more explicit, system of algebraic relations; it implies the system of associators.

We focus here on the combinatorial properties of another system of relations, the "double shuffles", which expresses some elementary series and integrals manipulations. We show that it shares an important property with associators and "motivic" relations, is implied by the latter and defines a polynomial algebra over $\mathbf{Q}$ (Écalle's theorem). We obtain these results for more general numbers: values of Goncharov's multiple polylogarithms at roots of unity.

\section{Introduction}

Les valeurs aux entiers strictement positifs des fonctions zêta multiples

$$
\zeta\left(s_{1}, \ldots, s_{r}\right):=\sum_{n_{1}>n_{2}>\cdots>n_{r}>0} 1 / n_{1}^{s_{1}} n_{2}^{s_{2}} \cdots n_{r}^{s_{r}}
$$

fournissent des solutions réelles de problèmes de nature purement algébrique, souvent dans le domaine de l'algèbre quantique. Déjà considérées par Euler dans le cas $r=2$, elles sont portées à l'attention du public par Zagier [39] au moment où elles apparaissent comme les coefficients de l'associateur de Drinfel'd $\Phi_{\mathrm{KZ}}[13,31]$. Plus récemment, Kontsevitch les a retrouvées dans son théorème de formalité [30]. On ne considère dans cette article que ces valeurs, que l'on appellera simplement «zêta multiples».

Dans une série d'articles [19-21], Goncharov s'intéresse à des objets plus généraux, les polylogarithmes multiples. Ce sont les fonctions de $r$ variables complexes définies dans le polydisque unité par : 


$$
\mathrm{L}_{s_{1}, \ldots, s_{r}}\left(z_{1}, \ldots, z_{r}\right):=\sum_{n_{1}>0}\left(\sum_{n_{1}>n_{2}>\cdots>n_{r}>0} \frac{z_{1}^{n_{1}} z_{2}^{n_{2}} \cdots z_{r}^{n_{r}}}{n_{1}^{s_{1}} n_{2}^{s_{2}} \cdots n_{r}^{s_{r}}}\right),
$$

où $s_{1}, \ldots, s_{r}$ sont des entiers strictement positifs. Ces séries entières sont de rayon de convergence 1 et ne divergent sur le bord du polydisque que pour $\left(s_{1}, z_{1}\right)=(1,1)$. Les zêta multiples ne sont que les valeurs de ces fonctions lorsque les $z_{i}$ valent tous 1 .

Les valeurs des polylogarithmes multiples sont aussi celles d'intégrales itérées du type

$$
\mathrm{I}_{[0,1]}\left(a_{1}, \ldots, a_{p}\right)=\int_{0 \leq t_{p} \leq \cdots \leq t_{1} \leq 1} \bigwedge_{i=1}^{p} \omega_{a_{i}}\left(t_{i}\right),
$$

avec $\omega_{a}(t)=d t /\left(a^{-1}-t\right)$ pour $a \neq 0$ et $\omega_{0}(t)=d t / t$.

Dans le cas des zêta multiples, c'est à Kontsevitch que l'on doit la formule reliant ces deux types d'objets. En convenant que $0^{k}$ désigne la séquence formée du chiffre 0 répété $k$ fois, elle s'écrit :

$$
\zeta\left(s_{1}, \ldots, s_{r}\right)=\mathrm{I}_{[0,1]}\left(0^{s_{1}-1}, 1,0^{s_{2}-1}, 1, \ldots, 0^{s_{r}-1}, 1\right),
$$

et Goncharov l'a généralisée aux polylogarithmes multiples.

1.1. Relations de mélange, exemples. - Notre propos est la combinatoire des relations de «double mélange» des valeurs des polylogarithmes multiples. On se limitera au cas où les $z_{i}$ parcourent le groupe $\boldsymbol{\mu}_{n}(\mathbf{G})$ des racines $n^{\text {èmes }}$ l'unité, $n$ étant fixé. Ces relations proviennent de manipulations formelles élémentaires, sur les écritures (1.b) et (1.c).

Par les séries entières, on obtient par exemple

$$
\begin{aligned}
\mathrm{L}_{s_{1}}\left(z_{1}\right) \mathrm{L}_{s_{2}}\left(z_{2}\right) & =\sum_{n_{1}, n_{2}>0} \frac{z_{1}^{n_{1}} z_{2}^{n_{2}}}{n_{1}^{s_{1}} n_{2}^{s_{2}}}=\left(\sum_{n_{1}>n_{2}>0}+\sum_{n_{2}>n_{1}>0}+\sum_{n_{1}=n_{2}>0}\right) \frac{z_{1}^{n_{1}} z_{2}^{n_{2}}}{n_{1}^{s_{1}} n_{2}^{s_{2}}} \\
& =\mathrm{L}_{s_{1}, s_{2}}\left(z_{1}, z_{2}\right)+\mathrm{L}_{s_{2}, s_{1}}\left(z_{2}, z_{1}\right)+\mathrm{L}_{s_{1}+s_{2}}\left(z_{1} z_{2}\right),
\end{aligned}
$$

ce qui se généralise au produit de deux séries quelconques du type (1.b).

D’un autre coté, le produit de deux simplexes se décompose en union de simplexes, ce qui fournit la relation de mélange des intégrales itérées :

$$
\mathrm{I}_{[0,1]}\left(a_{1}, \ldots, a_{p}\right) \mathrm{I}_{[0,1]}\left(b_{1}, \ldots b_{q}\right)=\sum_{\sigma \in \mathfrak{S}_{p, q}} \mathrm{I}_{[0,1]}\left(a_{\sigma^{-1}(1)}, \ldots, a_{\sigma^{-1}(i)}\right) .
$$

Dans cette formule, $\mathfrak{S}_{p, q}$ désigne l'ensemble des $(p, q)$-battages ${ }^{(1)}$ : les permutations de $\{1, \ldots, p+q\}$ qui sont croissantes sur $\{1, \ldots, p\}$ et $\{p+1, \ldots, q\}$.

(1) En anglais, les shuffles ; «mélange » est un terme moins précis. 
On a donc deux systèmes de relations dont la combinatoire semble similaire. On leur adjoindra la relation de régularisation, qui provient de l'annulation formelle de certaines divergences. Les arguments qu'on utilise pour l'obtenir sont empruntés à Boutet de Monvel [4]. On essaie d'en préciser l'historique.

On forme ainsi le système DMR (Doubles Mélanges \& Régularisation). Une conjecture de Kontsevitch et Zagier prévoit qu'il est complet dans le cas $n=1$, i.e. qu'il engendre toutes les relations algébriques entre zêta multiples. Les valeurs aux racines de l'unité satisfont également à d'autres relations, distribution et poids un, qu'on regroupe dans le système DMRD. Goncharov nous a dit qu'il n'était pas complet en général, mentionnant un contre-exemple pour $n=25$.

$\mathrm{Au}$ cours de la section 2, on donne une description duale du système DMRD :

Étant donné un sous-groupe fini multiplicatif $\Gamma$ de $\mathbf{G}^{*}$, on considère un alphabet $\mathrm{X}_{\Gamma}$ dont les éléments, notés $x_{\sigma}$, sont indexés par $\Gamma \cup\{0\}$. Les relations DMRD s'expriment de manière compacte sur la série génératrice non-commutative

$$
\mathscr{I}=\sum_{p \in \mathbf{N}, a_{1}, \ldots, a_{p} \in \Gamma \cup\{0\}} \mathrm{I}_{[0,1]}\left(a_{1}, \ldots, a_{p}\right) x_{a_{1}} \cdots x_{a_{p}},
$$

qui n'est autre, lorsque $\Gamma=\{1\}$, que le $\Phi_{\mathrm{KZ}}$ de Drinfel'd. ${ }^{(2)}$ La définition de $\mathscr{I}$ nécessite de «régulariser» les intégrales itérées divergentes, c'est-à-dire de leur donner un sens convenable. Cela sera fait globalement sur les séries génératrices.

On a choisi d'établir directement les relations DMRD dans cette optique duale, cela n'étant guère plus long qu'un rappel de la présentation habituelle et la preuve de l'équivalence. Cela nous a naturellement conduits à détailler les démonstrations de nombre de propriétés élémentaires.

1.2. Aspects motiviques. - Les intégrales itérées du type (1.c) ont un sens motivique qui explique une grande partie des propriétés algébriques des zêta multiples. On en donne ici un bref aperçu, renvoyant à [9,21,11] pour un exposé complet.

Soit $\mathrm{V}=\mathbf{G}_{\mathrm{m}} \backslash \boldsymbol{\mu}_{n}=\mathbf{P}^{1} \backslash\left(\{0, \infty\} \cup \boldsymbol{\mu}_{n}\right)$, vu comme schéma sur un corps cyclotomique $\mathrm{F}$ de degré $n$ et choisissons un plongement complexe $\iota$ de $\mathrm{F}$. Le groupoïde fondamental motivique de $\mathrm{V}$, défini par Goncharov [21], est un système de schémas en la catégorie tannakienne des motifs de Tate mixtes sur $\mathscr{O}_{\mathrm{F}, \mathrm{S}}$ de loc. cit. Il a les images décrites par Deligne dans [9] par les foncteurs fibres de réalisation $\omega_{\mathrm{B}, \iota}$ (de Betti, relativement à $\iota$ ) et $\omega_{\mathrm{DR}}$ (de de Rham). En réalisation de de Rham, $\pi_{1}^{\mathrm{DR}}(\mathrm{V} ; a, b)$ est indépendant des points-base $a$ et $b$ et s'identifie aux exponentielles de Lie formées sur $\mathrm{X}:=\mathrm{X}_{\mu_{n}(\mathrm{~F})}$. On a une $\mathbf{Q}$-structure canonique $\omega_{\mathrm{gr}}$ sur le foncteur-fibre $\omega_{\mathrm{DR}}[11]$.

(2) Nos notations et celles de $[13, \S 5]$ se correspondent par $x_{0}=\mathrm{A}, x_{1}=-\mathrm{B}$. 
La série $\mathscr{I}$ s'interprète comme l'image du chemin $[0,1]$ par l'isomorphisme de comparaison

$$
\pi_{1}^{\mathrm{B}, \iota}(\mathrm{V} ;(0,1),(1,0))(\mathbf{G}) \longrightarrow \pi_{1}^{\mathrm{gr}}(\mathrm{V} ;(0,1),(1,0))(\mathbf{G}),
$$

où $(0,1)$ et $(1,0)$ sont des points-base tangentiels de Deligne en 0 et 1 .

Par la théorie générale des catégories tannakiennes, les isomorphismes entre les foncteurs-fibre $\omega_{\mathrm{B}}$ et $\omega_{\mathrm{gr}}$ forment un schéma affine sur $\mathbf{Q}$, torseur sous le schéma en groupes $\mathscr{G}_{\mathrm{gr}}$ des automorphismes de $\omega_{\mathrm{gr}}$. Ce dernier se décompose en $\mathscr{G}_{\mathrm{gr}}=\mathbf{G}_{\mathrm{m}} \ltimes \mathscr{U}_{\mathrm{gr}}$, où $\mathscr{U}$ est le noyau de l'action sur le motif de Tate $\mathbf{Q}(1)$. Par définition, les actions de $\mathscr{G}_{\mathrm{gr}}$ et $\mathscr{U}_{\mathrm{gr}}$ commutent à toute flèche de la forme $\omega_{\mathrm{gr}}(f)$.

Soient $\mathrm{G}$ et $\mathrm{H}$ deux éléments de $k\langle\langle\mathrm{X}\rangle\rangle$. On étudie en 3.1 le produit $\mathrm{G} \circledast \mathrm{H}$, défini si le terme constant de $\mathrm{G}$ est 1 . Ge produit fait des séries à terme constant 1 de $k\langle\langle\mathrm{X}\rangle\rangle$ un groupe pro-unipotent, noté $\mathrm{MT}(k)$, qui agit sur $k\langle\langle\mathrm{X}\rangle\rangle$. Cette opération apparaît, sous diverses formes, chez Drinfel'd, Goncharov et Ihara. Le groupe MT est muni d'une action du groupe multiplicatif $\mathbf{G}_{\mathrm{m}}$ comme suit : $\lambda$ transforme $\mathrm{G} \in \mathrm{MT}$ en multipliant sa composante homogène de degré $n$ par $\lambda^{n}$. Comme les degrés sont positifs, ceci s'étend en une action du monoïde multiplicatif $\left(\mathbf{A}^{1}, \times\right)$ sur MT. On note $\tau$ l'application canonique $\mathbf{G}_{\mathrm{m}} \rightarrow \mathbf{G}_{\mathrm{m}} \ltimes \mathrm{MT}$.

Il est expliqué en détail dans [11] comment l'action de $\mathscr{U}_{\mathrm{gr}}$ sur $\pi_{1}^{\mathrm{gr}}(\mathrm{V} ;(0,1)$, $(1,0))$ se factorise par MT, i.e. se fait par la loi $\circledast$. Deligne construit un sousschéma $\mathrm{M}^{\iota}$ de $\mathbf{A}^{1} \times \mathrm{MT}$ dont $(2 i \pi, \mathscr{I})$ est un point complexe, traduisant les relations algébriques entre valeurs aux racines de l'unité des polylogarithmes multiples et $2 i \pi$ qui se déduisent de la comparaison Betti-de Rham.

Une variante de la conjecture des périodes de Grothendieck prévoit que ce système de relations est complet. Le tout satisfait à la propriété (M) suivante :

Définition 1.2.1. - Soit $\mathrm{P}$ un sous-schéma de $\mathbf{A}^{1} \times \mathrm{MT}$ et soit $p$ la première projection. On considère les fibres $\mathrm{P}_{\lambda}$ de $p$ comme incluses dans $\mathrm{MT}$ et on identifie les schémas $\mathbf{G}_{\mathrm{m}} \times \mathrm{MT}$ et $\mathbf{G}_{\mathrm{m}} \ltimes \mathrm{MT}$ par $(\lambda, u) \mapsto u \tau(\lambda)$. On dit que $\mathrm{P}$ a la propriété $(M)$ si et seulement si :

$-\mathscr{I} \in \mathrm{P}_{2 i \pi}(\mathbf{G})$

- Il existe $\delta \in \mathrm{P}(\mathbf{Q})$ et un sous-groupe $\mathrm{I}$ de $\mathrm{MT}$ stable par $\mathbf{G}_{\mathrm{m}}$ tels que $p^{-1}\left(\mathbf{G}_{\mathrm{m}}\right)=$ $\left(\mathbf{G}_{\mathrm{m}} \ltimes \mathrm{I}\right) \delta$.

- De plus, l'espace des $\mathrm{I}\left(\tau(\lambda) \delta \tau(\lambda)^{-1}\right) \tau(\lambda)$ est $\mathrm{P}$, où la conjugaison par $\tau(\lambda)$ est à interpréter comme l'action du monö̈de multiplicatif $\mathbf{A}^{1}$ sur $\mathrm{MT}$. On a donc $\mathrm{I}=\mathrm{P}_{0}$.

Le groupe $\mathrm{M}_{0}$ est par construction l'image de $\mathscr{U}_{\mathrm{gr}}$ dans MT. On ne connaît pas de description de $M$ par un système explicite d'équations. Dans le cas des zêta multiples, i.e. $\mathrm{V}=\mathbf{P}^{1} \backslash\{0,1, \infty\}$, la variété Ass des associateurs de Drinfel'd, (le $M$ de [13]), est un candidat. Les équations qui la définissent sont liées aux conditions de cohérence de Mac-Lane et elle a la propriété (M) [13, props. 5.5 
\& 5.9]. La nature motivique de Ass fait peu de doute, apparaissant clairement dans la présentation de Bar-Natan [2] : on peut interpréter ses catégories $\widehat{\mathrm{PAB}}$ et $\widehat{\mathrm{PACD}}$ comme les «tours» Betti et de Rham des groupoïdes fondamentaux des espaces de configuration de $n$ points sur la droite affine, restreints à un jeu de points-base tangentiels. Le groupe $\mathrm{Ass}_{0}$ est la variante de de Rham du groupe de Grothendieck-Teichmüller ; Drinfel'd le note $\mathrm{GRT}_{1}$. L'étude de Ass, et particulièrement la recherche d'associateurs rationnels est en soi un problème important, $\mathrm{du}$ fait des nombreuses applications $[38,16,1,6]$.

Le versant $\ell$-adique de ces objets a été étudié indépendamment par Ihara $[29,26,28]$, sous une autre terminologie : «stable derivation algebra» au lieu de $\mathfrak{g r t}_{1}$. Hain et Matsumoto [22] ont récemment accompli des progrès dans cette direction.

1.3. Résultat principal. - On décrit explicitement le groupe MT dans la section 3 et on en étudie les propriétés les plus immédiates, notamment différentielles. On définit ensuite pour tout groupe commutatif fini $\Gamma$ un sous-schéma DMR de MT qui traduit les relations DMR, dans le cas où $\Gamma$ est un sous-groupe de $\mathbf{G}^{*}$. Les relations de poids un dépendent du choix d'un plongement $\iota: \Gamma \rightarrow \mathbf{C}^{*}$, et amènent à définir des sous-schémas $\mathrm{DMR}^{\iota}$ et $\mathrm{DMRD}^{\iota}$ de $\mathbf{A}^{1} \times \mathrm{MT}$, dont $(2 i \pi, \mathscr{I})$ est un point complexe.

Ceci permet alors d'énoncer le résultat principal de cet article : DMR ${ }^{\iota}$ et $\mathrm{DMRD}^{\iota}$ ont la propriété $(\mathrm{M})$. On utilise la forme équivalente annoncée dans [34, 35].

Qu'un couple $(\lambda, \Phi)$ soit un point de $\mathrm{DMR}^{\iota}$ s'exprime par une dépendance entre $\lambda$ et les coefficients de certains mots dans $\Phi$. Ces derniers ont une autre interprétation : ce sont les cas exceptionnels d'une propriété combinatoire de l'espace tangent $\mathfrak{d} \mathfrak{m r}$ à $\underline{\mathrm{DMR}}$ au voisinage de la solution évidente 1. Celle-ci jouera un rôle important dans la preuve du théorème principal. On pourra a posteriori la considérer comme l'origine du rôle particulier de $2 i \pi$, du point de vue des relations DMR.

1.4. Preuve. - La démonstration se fait en deux étapes. D'abord, on prouve que les fibres $\mathfrak{d} \mathfrak{m} \mathfrak{r}_{0}$ et $\mathfrak{d} \mathfrak{m r} \mathfrak{d}_{0}$ sont des sous-algèbres de Lie de $\mathfrak{m t}$, l'algèbre de Lie de MT, et que les $\mathrm{DMR}_{\lambda}^{\iota}$ sont stables par l'action de l'exponentielle, au sens de MT, de $\mathfrak{d m r}_{0}$. C'est l'objet de la section 4. La démonstration repose sur l'enchaînement de plusieurs miracles combinatoires, et l'auteur doit bien avouer ne pas avoir d'argument plus direct à proposer : la nature motivique des relations du type (1.1.5) n'est pas vraiment comprise.

$\mathrm{Au}$ cours de la section 5, on complète la démonstration du théorème en montrant que l'action de $\exp \left(\mathfrak{d m} \mathfrak{r}_{0}\right)(k)$ sur $\operatorname{DMR}_{\lambda}^{\iota}(k)$ est transitive pour tout $k$. Cela résulte de la nature d'espace tangent de $\mathfrak{d} \mathfrak{m} \mathfrak{r}_{0}$ et de l'existence d'une solu- 
tion particulière $: \mathscr{I}$. On utilise une méthode d'approximations successives inspirée de [2], qu'on développe dans un cadre un peu plus général.

On étudie ensuite quelques conséquences directes du théorème. Notamment, on exhibe certains éléments irréductibles de $\mathfrak{d} \mathfrak{m} \mathfrak{r} \mathfrak{d}_{0}$ égaux, dans le cas des zêta multiples, à ceux de $\mathfrak{g r t}_{1}$ définis par Drinfel'd [13]. Dans tous les cas, ils engendrent l'algèbre de Lie de $\mathrm{M}_{0}$ dans la description de Deligne [11]. Autrement dit, les relations motiviques impliquent les relations DMRD.

1.5. Remerciements. - Les plus vifs sont adressés à Pierre Cartier, qui a encadré ce travail, et à Pierre Deligne pour ses patientes explications. La présentation de la section 2 profite directement des raccourcis de [10].

Merci également à S. Aicardi, J. Bellaïche, L. Boutet de Monvel, F. Digne, B. Enriquez, A. Goncharov, I. Marin, J.-C. Novelli, M. Petitot et C. Reutenauer.

Ce travail a été effectué alors que l'auteur préparait une thèse à l'université de Picardie-Jules-Verne et était membre du département de mathématiques de l'École Normale Supérieure. L'auteur bénéficie actuellement d'une bourse postdoctorale du réseau TMR «Arithmetic Algebraic Geometry» à l'université de Münster, où la rédaction a été achevée.

\section{TABLE DES MATIÈRES}

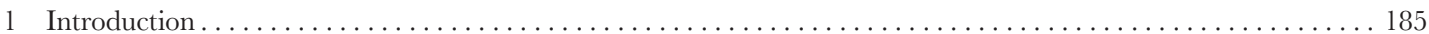

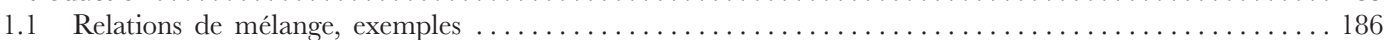

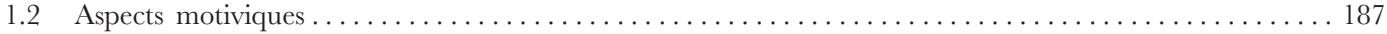

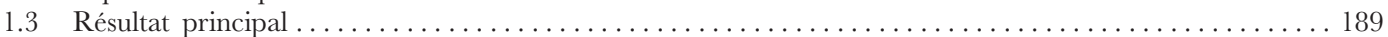

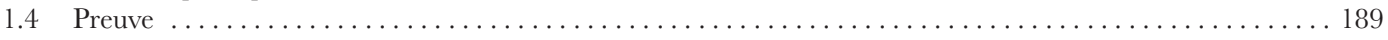

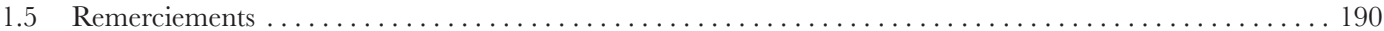

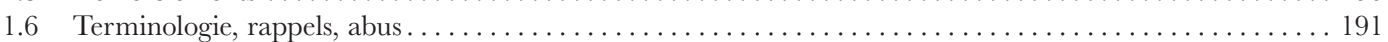

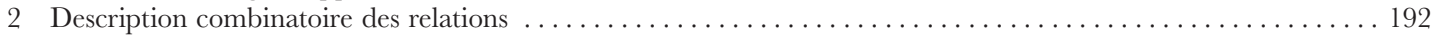

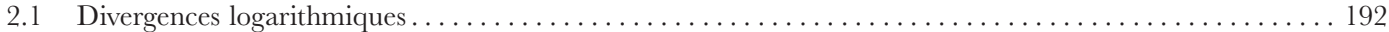

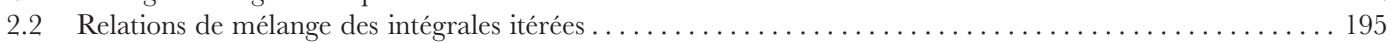

2.3 Relation de mélange des sommes itérées $\ldots \ldots \ldots \ldots \ldots \ldots \ldots \ldots \ldots \ldots \ldots \ldots \ldots \ldots \ldots \ldots \ldots \ldots \ldots$

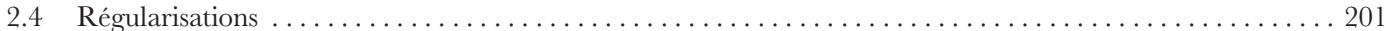

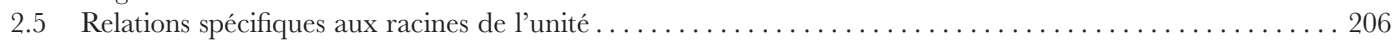

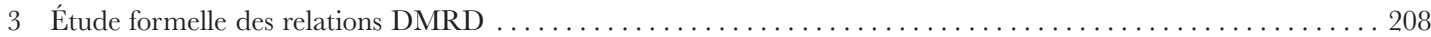

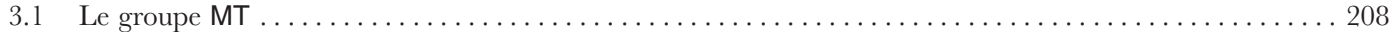

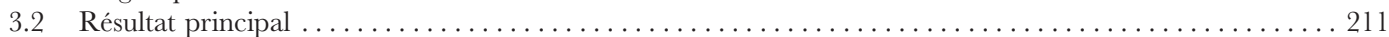

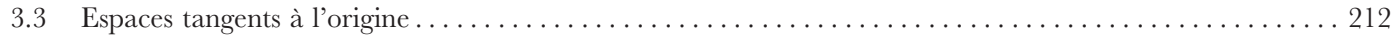

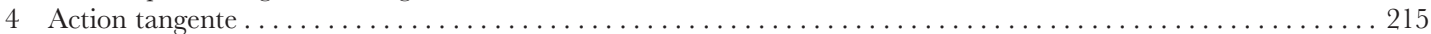

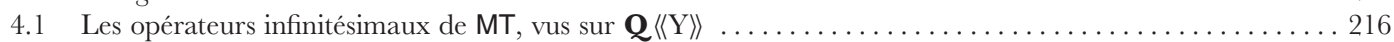

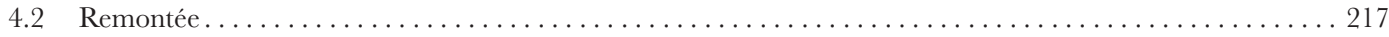

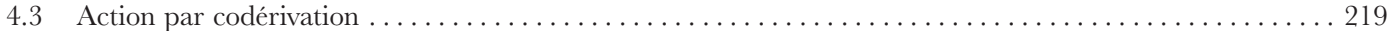

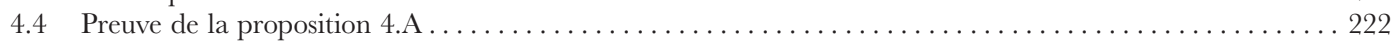

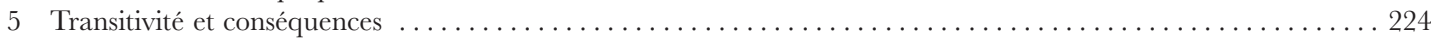

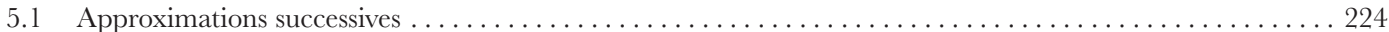

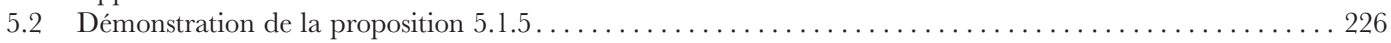

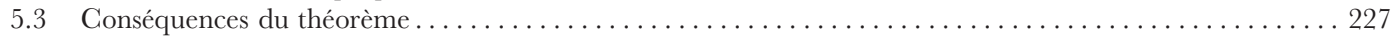

Index des notations . . . . . . . . . . . . . . . . . . . . . . . . . . . . . . . . . . . . . . . . . . . . . . 229

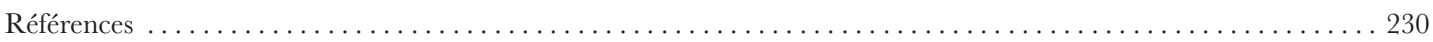


1.6. Terminologie, rappels, abus. - Les anneaux sont supposés commutatifs et unifères, les algèbres sont associatives et unifères ; les cogèbres sont coassociatives et coünifères. Un $\mathbf{Q}$-anneau est un anneau contenant $\mathbf{Q}$. Le groupe à un élément est noté $\mathbf{1}$. On fixe une clôture algébrique $\mathbf{G}$ de $\mathbf{R}$.

On adopte le point de vue fonctoriel sur les schémas, utilisé notamment par Demazure et Gabriel [12] : un schéma affine sur $\mathbf{Q}$ est un foncteur représentable de la catégorie des $\mathbf{Q}$-anneaux. C'est une limite projective de schémas affines de type fini, i.e. algébriques. La lettre $k$ désigne en général un $\mathbf{Q}$-anneau quelconque, qui sert de variable pour ces foncteurs.

En l'absence de précisions, les graduations sont des $\mathbf{N}$-graduations. On utilise implicitement le produit tensoriel gradué et le produit tensoriel complété. Ce dernier est noté $\widehat{\otimes}$ si l'on tient à préciser.

Un $\mathbf{Q}$-espace vectoriel gradué $\mathrm{V}$ dont chaque composante homogène $\mathrm{V}_{n}$ de degré $n$ est de dimension finie donne lieu à un schéma vectoriel $k \mapsto \mathrm{V} \widehat{\otimes} k=\prod_{n} \mathrm{~V}_{n} \otimes k$. On le notera simplement $\mathrm{V}$, utilisant $\mathrm{V}(k)$ si nécessaire. La $\mathbf{Q}$-algèbre correspondante est l'algèbre symétrique du dual gradué de $\mathrm{V}$ (voir [12, p. 147] pour le cas usuel).

On dira qu'un élément $x$ d'une cogèbre $(\mathrm{C}, \varepsilon, \Delta)$ est diagonal s'il vérifie $\Delta x=$ $x \otimes x$ et $\varepsilon(x)=1$. Cette terminologie a été jugée préférable à l'utilisation répétée de «group-like». Si C est une bigèbre graduée, telle que le noyau de $\varepsilon$ est à degrés strictement positifs, l'application exponentielle est une bijection de l'ensemble des éléments primitifs du complété $\widehat{\mathrm{C}}$ sur l'ensemble des éléments diagonaux de $\widehat{\mathrm{C}}$.

Pour un ensemble $Z$, on désigne respectivement par $k\langle Z\rangle$ et $\mathfrak{L i b}_{k}(Z)$ l'algèbre associative libre et l'algèbre de Lie libre sur Z, à coefficients dans $k$. Dans tous les cas que l'on considérera, une graduation convenable permettra de définir les algèbres filtrées complètes correspondantes, notées $k\langle\langle\mathrm{Z}\rangle\rangle$ et $\widehat{\mathfrak{L i b}}_{k}(\mathrm{Z})$ et de traiter leurs éléments comme des séries formelles. On renvoie au livre de Reutenauer [37] pour un exposé et une bibliographie récents à propos des algèbres de Lie libres.

Pour ces objets libres, on qualifiera de substitution un morphisme dans la catégorie appropriée défini par son action sur les générateurs.

Pour $w_{1}, \ldots, w_{n}$, mots en $Z$, et $\Phi \in k\langle\langle Z\rangle\rangle^{\widehat{\otimes} n}$, la notation $\left(\Phi \mid w_{1} \otimes \cdots \otimes w_{n}\right)$ désigne le coefficient de $w_{1} \otimes \cdots \otimes w_{n}$ dans $\Phi$ et est étendue à tout élément de $k\langle Z\rangle^{\otimes n}$ par linéarité sur le membre de droite.

Une $\mathbf{Q}$-algèbre de Lie $\mathfrak{g}$ est considérée comme incluse dans son algèbre enveloppante universelle $U \mathfrak{g}$. C'est une bigèbre de Hopf pour le coproduit $\Delta$ pour lequel les éléments de $\mathfrak{g}$ sont primitifs, avec la coünité $\varepsilon$ donnée par $\mathfrak{g} \subset$ ker $\varepsilon$ et l'antipode $\mathrm{S}$ par $\mathrm{S}(x)=-x$ pour tout $x \in \mathfrak{g}$. Si $\mathfrak{g}$ est graduée, à degrés strictement positifs, et à composantes de dimension finie, on note $\widehat{U_{k} \mathfrak{g}}$ le complété à coefficients dans $k$, munie de la structure de bigèbre obtenue par l'extension continue des scalaires $\widehat{\otimes} k$.

On utilisera fréquemment, sous l'appellation «test sur les générateurs », la propriété immédiate suivante : un morphisme d'algèbres (resp. une dérivation) entre deux 
bigèbres est un morphisme de cogèbres (resp. une codérivation) si l'identité appropriée est vraie sur un système de générateurs. Par exemple, une dérivation de $U \mathfrak{g}$ qui laisse $\mathfrak{g}$ stable est aussi une codérivation.

\section{Description combinatoire des relations}

2.1. Divergences logarithmiques. - Notre but est ici de décrire les étapes de Boutet de Monvel qui nous permettront d'établir la relation de régularisation.

On note $\mathrm{D}$ le disque ouvert unité de $\mathbf{C}$ et $\mathscr{H}(\mathrm{D})$ l'algèbre des fonctions holomorphes sur D. La lettre $t$ désigne une variable formelle.

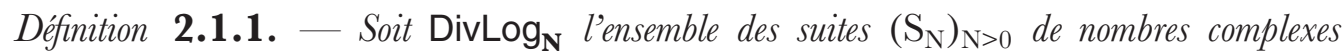
admettant un développement asymptotique du type

$$
\mathrm{S}_{\mathrm{N}}=\operatorname{As}\left(\left(\mathrm{S}_{\mathrm{N}}\right)\right)(\log (\mathrm{N}))+\mathrm{O}\left(\log ^{\alpha}(\mathrm{N}) / \mathrm{N}\right),
$$

avec $\operatorname{As}\left(\left(\mathrm{S}_{\mathrm{N}}\right)\right) \in \mathbf{C}[t]$ et $\alpha \in \mathbf{N}$.

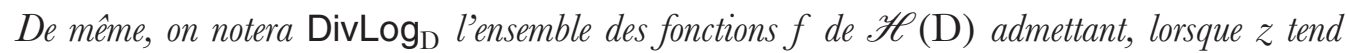
vers 1 dans $\mathrm{D} \cap \mathbf{R}$, un développement du type

$$
f(z)=\operatorname{As}(f)(-\log (1-z))+\mathrm{O}\left(\left|(1-z) \log ^{\alpha}(1-z)\right|\right)
$$

avec $\operatorname{As}(f) \in \mathbf{G}[t]$ et $\alpha \in \mathbf{N}$.

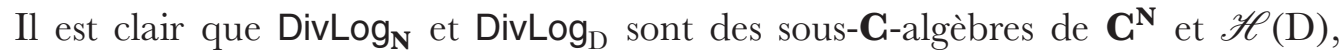
respectivement ; que les polynômes $\operatorname{As}\left(\left(\mathrm{S}_{\mathrm{N}}\right)\right)$ et $\operatorname{As}(f)$ sont uniquement déterminés par ces conditions et que les applications As ainsi définies sont toutes deux des morphismes d'algèbres, grâce à la nature du reste.

2.1.2. À une suite $\left(\mathrm{S}_{\mathrm{N}}\right)_{\mathrm{N}>0}$, faisons correspondre l'unique série entière $\sum_{n>0} u_{n} z^{n}$ telle que $\mathrm{S}_{\mathrm{N}}=\sum_{n=1}^{\mathrm{N}} u_{n}$.

Proposition 2.1.3. - Si $\left(\mathrm{S}_{\mathrm{N}}\right)_{\mathrm{N}>0}$ appartient à DivLog $\operatorname{No}_{\mathbf{N}}$ et $\operatorname{As}\left(\left(\mathrm{S}_{\mathrm{N}}\right)\right)=0$, la série entière associée définit une fonction $f$ de $\operatorname{DivLog}_{\mathrm{D}}$, vérifiant $\operatorname{As}(f)=0$.

Démonstration. - On suppose donc qu'on a un entier $\alpha$ tel que

$$
\mathrm{S}_{\mathrm{N}}=: \sum_{n=1}^{\mathrm{N}} u_{n}=\mathrm{O}\left(\log ^{\alpha}(\mathrm{N}) / \mathrm{N}\right)
$$


DOUBLES MÉLANGES DES POLYLOGARITHMES MULTIPLES AUX RACINES DE L'UNITÉ 193

Effectuons une transformation d'Abel :

$$
\begin{aligned}
f(z) & =\sum_{n>0} u_{n} z^{n}=\sum_{\mathrm{N}>0}\left(\sum_{n=1}^{\mathrm{N}} u_{n}\right)\left(z^{\mathrm{N}}-z^{\mathrm{N}+1}\right) \\
& =(1-z) \sum_{\mathrm{N}>0} z^{\mathrm{N}} \mathrm{O}\left(\log ^{\alpha}(\mathrm{N}) / \mathrm{N}\right) .
\end{aligned}
$$

Il suffit donc d'obtenir l'existence de $\beta>0$ tel que

$$
\sum_{\mathrm{N}>0} z^{\mathrm{N}} \log ^{\alpha}(\mathrm{N}) / \mathrm{N}=\mathrm{O}\left(\left|\log (1-z)^{\beta}\right|\right)
$$

Découpons la somme en deux, autour de $1 /(1-z)$. En majorant $z$ par 1 , on obtient

$$
\sum_{0<\mathrm{N}<(1-z)^{-1}} z^{\mathrm{N}} \log ^{\alpha} \mathrm{N} / \mathrm{N} \leq \int_{1}^{(1-z)^{-1}} \frac{d t \log ^{\alpha} t}{t}=(-\log (1-z))^{\alpha+1} /(\alpha+1) .
$$

La fonction $\log ^{\alpha}(t) / t$ est décroissante au voisinage de $+\infty$. Pour $z$ suffisamment proche de 1 , et $\mathrm{N} \geq(1-z)^{-1}$, on a donc $\log ^{\alpha}(\mathrm{N}) / \mathrm{N} \leq(-\log (1-z))^{\alpha}(1-z)$, dont on déduit

$$
\begin{aligned}
\sum_{\mathrm{N} \geq(1-z)^{-1}} z^{\mathrm{N}} \log ^{\alpha}(\mathrm{N}) / \mathrm{N} & \leq(-\log (1-z))^{\alpha}(1-z) \sum_{\mathrm{N} \geq(1-z)^{-1}} z^{\mathrm{N}} \\
& \leq z^{1 / 1-z}(-\log (1-z))^{\alpha} .
\end{aligned}
$$

Comme $z^{1 / 1-z}$ tend vers $\exp (-1)$, ceci donne le résultat, avec $\beta=\alpha+1$.

2.1.4. La série harmonique $\mathrm{H}_{\mathrm{N}}=\sum_{n=1}^{\mathrm{N}} 1 / n$ appartient à DivLog $\mathbf{N}$. On a $\mathrm{As}\left(\mathrm{H}_{\mathrm{N}}\right)=\gamma+t$, où $\gamma$ est la constante d'Euler. Le morphisme d'algèbres As : $\operatorname{DivLog}_{\mathbf{N}} \rightarrow \mathbf{C}[t]$ est donc surjectif. De même, en considérant la fonction $\mathrm{L}_{1}: z \mapsto$ $-\log (1-z)$, on voit que As : DivLog $\rightarrow \mathbf{C}[t]$ est également surjectif.

Soient $k$ un entier positif et $\left(\mathrm{H}_{k, \mathrm{~N}}\right)_{\mathrm{N}>0}$ la suite associée à la fonction $\left(\mathrm{L}_{1}\right)^{k} / k$ ! Il sera prouvé au paragraphe 2.4.8 que $\left(\mathrm{H}_{k, \mathrm{~N}}\right)_{\mathrm{N}>0}$ appartient à DivLog $\mathbf{N}_{\mathbf{N}}$ et que le polynôme $\operatorname{As}\left(\left(\mathrm{H}_{k, \mathrm{~N}}\right)_{\mathrm{N}>0}\right)$ est de degré $k$. Pour tout $\left(\mathrm{S}_{\mathrm{N}}\right)_{\mathrm{N}>0} \in \operatorname{DivLog}_{\mathbf{N}}$, il existe donc une combinaison linéaire de $\left(\mathrm{S}_{\mathrm{N}}\right)$ et des $\left(\mathrm{H}_{k, \mathrm{~N}}\right)$ qui est dans le noyau de As. On en déduit, grâce à la proposition 2.1.3, le résultat ci-dessous, qui ne servira pas avant 2.4.10 :

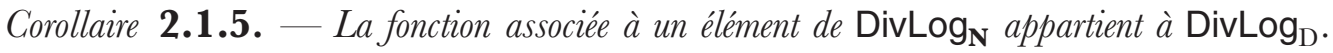
Il existe une application $\mathbf{C}$-linéaire $\mathrm{cmp}$ faisant commuter le diagrame ci-dessous.

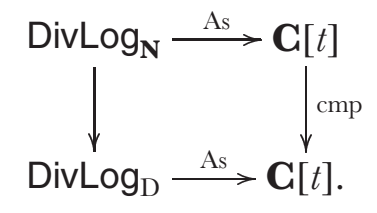


Proposition 2.1.6 (Itération). - Soit s un entier strictement positif, z dans le disque unité fermé de $\mathbf{G}$ et $\left(\mathrm{S}_{\mathrm{N}}\right)_{\mathrm{N}>0} \in \operatorname{DivLog}_{\mathrm{N}}$, La suite $\left(\mathrm{T}_{\mathrm{N}}\right)_{\mathrm{N}>0}$

$$
\mathrm{T}_{\mathrm{N}}:=\sum_{0<n<\mathrm{N}} z^{n} \mathrm{~S}_{n} / n^{s}
$$

est encore dans DivLog ${ }_{\mathrm{N}}$. La dérivée du polynôme $\mathrm{As}\left(\mathrm{T}_{\mathrm{N}}\right)$ est $\mathrm{As}\left(\mathrm{S}_{\mathrm{N}}\right)$. La suite $\mathrm{T}_{\mathrm{N}}$ est convergente si $(s, z) \neq(1,1)$.

Démonstration. - On a donc un polynôme $\mathrm{P}$ tel que $\mathrm{S}_{n}=\mathrm{P}(\log (n))+\mathrm{O}\left(\log ^{\alpha}(n) / n\right)$ Dans un premier temps, on majore $|z|$ par 1 .

Dans le cas $s \geq 2$, la convergence de $\mathrm{T}_{\mathrm{N}}$ est évidente. Il faut donc évaluer le reste de la série. Au pire, on a à faire à $\sum_{n>\mathrm{N}} \log ^{\beta}(n) / n^{2}$, où $\beta$ est le degré de $\mathrm{P}$. Ceci est équivalent à l'intégrale

$$
\mathrm{I}_{\beta}:=\int_{\mathrm{N}}^{+\infty} \log ^{\beta} x^{-2} d x
$$

qui vérifie $\mathrm{I}_{\beta}=\beta \log ^{\beta-1}(\mathrm{~N}) / \mathrm{N}+\beta \mathrm{I}_{\beta-1}$ avec $\mathrm{I}_{0}=1 / \mathrm{N}$.

Si $s=1$, on a un terme de la forme $\mathrm{O}\left(\log ^{\alpha}(n)\right) / n^{2}$, qui est traité comme cidessus, et le terme $\mathrm{P}(\log (n)) / n)$. Il suffit de traiter le cas $\mathrm{P}(t)=t^{k}$, pour tout $k \in \mathbf{N}$. obtient :

En mettant $\log (n+1)-\log (n)$ en facteur dans $\log ^{k+1}(n+1)-\log ^{k+1}(n)$, on

$$
\log ^{k}(n) / n=\left(\log ^{k+1}(n+1)-\log ^{k+1}(n)\right) /(k+1)+\mathrm{O}\left(\log ^{k}(n) / n^{2}\right) .
$$

La sommation du premier terme donne $\log ^{k+1}(\mathrm{~N}) /(k+1)$. Le reste est traité comme précédemment. Ceci prouve les deux premières assertions.

Comme $\mathrm{S}_{n} / n$ tend vers 0 , la convergence est dans le cas $s=1, z \neq 1$ une application classique de la transformation d'Abel.

2.1.7. Par définition, $\mathrm{L}_{s_{1}, \ldots, s_{r}}\left(z_{1}, \ldots, z_{r}\right)$ est la limite de la suite

$$
\mathrm{L}_{s_{1}, \ldots, s_{r}}^{\mathrm{N}}\left(z_{1}, \ldots, z_{r}\right):=\sum_{\mathrm{N}>n_{1}>n_{2}>\cdots>n_{r}>0} \frac{z_{1}^{n_{1}} z_{2}^{n_{2}} \cdots z_{r}^{n_{r}}}{n_{1}^{s_{1}} n_{2}^{s_{2}} \cdots n_{r}^{s_{r}}} .
$$

Corollaire 2.1.8. - Pour tous entiers strictement positifs $s_{1}, \ldots, s_{r}$ et tous $z_{1}, \ldots, z_{r}$ de module au plus 1 , la suite $\left(\mathrm{L}_{s_{1}, \ldots, s_{r}}^{\mathrm{N}}\left(z_{1}, \ldots, z_{r}\right)\right)_{\mathrm{N}>0}$ appartient à DivLog $\log _{\mathbf{N}}$. Elle est convergente si et seulement si $\left(s_{1}, z_{1}\right) \neq(1,1)$.

Démonstration. - La proposition 2.1.6 permet d'effectuer une récurrence sur $r$. 
DOUBLES MÉLANGES DES POLYLOGARITHMES MULTIPLES AUX RACINES DE L'UNITÉ 195

2.1.9. Remarques. - Boutet de Monvel considère des développements plus fins, du type $\sum_{i \geq 0} \mathrm{P}_{i}(\log \mathrm{N}) / \mathrm{N}^{i}$. Il aboutit à la jolie formule $\mathrm{cmp}=(d / d t)$ !, où la factorielle désigne le développement en série entière de la fonction $\Gamma$ d'Euler au voisinage de 1 .

Dans [33], on utilisait des restes en $\mathrm{O}\left(\mathrm{N}^{-\alpha-1}\right)$, avec $\alpha \in \mathbf{R}_{+}^{*}$, plus grossiers qu'ici. Les démonstrations pour les restes en $\log ^{\alpha}(\mathrm{N}) / \mathrm{N}$ sont celles de [10].

2.2. Relations de mélange des intégrales itérées

Définition 2.2.1. - Soient $a_{1}, \ldots, a_{p} \in \mathbf{G}$ et $\gamma:[0,1] \rightarrow \mathbf{G}$ un chemin. On considère l'intégrale éventuellement divergente :

$$
\mathrm{I}_{\gamma}\left(a_{1}, \ldots, a_{p}\right):=\int_{0}^{1}\left(\gamma^{*} \omega_{a_{1}}\right)\left(t_{1}\right) \int_{0}^{t_{1}}\left(\gamma^{*} \omega_{a_{2}}\right)\left(t_{2}\right) \cdots \int_{0}^{t_{p}}\left(\gamma^{*} \omega_{a_{p}}\right)\left(t_{p}\right),
$$

avec, pour tout $a \in \mathbf{C}$ :

$$
\omega_{a}(t):= \begin{cases}d t /\left(a^{-1}-t\right) & \text { si } a \neq 0 \\ d t / t & \text { si } s=0\end{cases}
$$

Lorsque $\gamma$ évite les singularités $a_{1}^{-1}, \ldots, a_{p}^{-1}$, cette intégrale est bien définie et ne dépend que de la classe d'homotopie, à extrémités fixées, de $\gamma$ dans $\mathbf{C} \backslash\left\{a_{1}^{-1}, \ldots, a_{p}^{-1}\right\}$. Pour $p=0$, elle vaudra 1 par convention.

Pour $(a, b) \in \mathbf{C}^{2}$, on notera $[a, b]$ le chemin $t \mapsto a+t(b-a)$.

Goncharov utilise une généralisation de la formule de Kontsevitch. En convenant que $0^{k}$ désigne la séquence formée du chiffre 0 répété $k$ fois, elle s'écrit :

Proposition 2.2.2. - Soient $r \in \mathbf{N}, s_{1}, \ldots, s_{r} \in \mathbf{N}^{*}$ et $z_{1}, \ldots, z_{r}$ des nombres complexes tels que $0<\left|z_{i}\right| \leq 1$. Pour tout $z$ du disque unité ouvert de $\mathbf{G}$, on a

$$
\mathrm{L}_{s_{1}, \ldots, s_{r}}\left(z z_{1}, z_{2}, \ldots, z_{r}\right)=\mathrm{I}_{[0, z]}\left(0^{s_{1}-1}, z_{1}, 0^{s_{2}-1}, z_{1} z_{2}, \ldots, 0^{s_{r}-1}, z_{1} z_{2} \cdots z_{r}\right) .
$$

2.2.3. Séries de Chen. - Soit $\Gamma$ un groupe commutatif fini, noté multiplicativement. On considère un alphabet $\mathrm{X}_{\Gamma}=\left\{\left(x_{\sigma}\right)\right\}$ indexé par $\Gamma \cup\{0\}$. Lorsqu'il n'y a pas d'ambiguité sur $\Gamma$, on écrira simplement $\mathrm{X}$ pour $\mathrm{X}_{\Gamma}$.

On appellera poids la graduation qui attribue à chaque élément de $\mathrm{X}$ le degré 1. Rappelons que le coproduit $\Delta$ de $\mathbf{Q}\langle\mathrm{X}\rangle$, vue comme bigèbre enveloppante de $\mathfrak{L i b}(\mathrm{X})$, est homogène et est donné par $\Delta x_{\sigma}=1 \otimes x_{\sigma}+x_{\sigma} \otimes 1$, pour tout $\sigma \in \Gamma \cup\{0\}$.

Supposons $\Gamma$ inclus dans $\mathbf{G}^{*}$. À tout chemin $\gamma$ sur $\mathbf{G}^{*} \backslash \Gamma$, on associe l'élément suivant de $\mathbf{C}\langle\langle\mathrm{X}\rangle\rangle$, appelé série de Chen :

$$
\mathscr{I}_{\gamma}:=\sum_{p \in \mathbf{N}, \sigma_{1}, \ldots, \sigma_{p} \in \Gamma \cup\{0\}} \mathrm{I}_{\gamma}\left(\sigma_{1}, \ldots, \sigma_{p}\right) x_{\sigma_{1}} \cdots x_{\sigma_{p}} .
$$

Proposition 2.2.4 (Relation de mélange). — L'élément $\mathscr{I}_{\gamma}$ de $(\mathbf{C}\langle\langle\mathrm{X}\rangle\rangle, \cdot, \Delta)$ est diagonal, pour tout chemin $\gamma$ sur $\mathbf{G}^{*} \backslash \Gamma$. 
C'est un fait bien connu, qui remonte aux années cinquante [7], et il en existe de nombreuses démonstrations. Une première méthode [36], est d'établir la formule (1.1.6), qui permet d'interpréter $\mathrm{I}_{\gamma}$ comme un morphisme d'algèbres à valeurs dans $\mathbf{G}$, depuis le dual gradué de $\mathbf{Q}\langle\mathrm{X}\rangle$ muni du produit de battage $\sqcup$; ce dernier est dual du coproduit $\Delta[37]$.

On peut aussi voir $\mathscr{I}_{[a, z]}$ comme solution de l'équation différentielle

$$
d \mathscr{I}_{[a, z]}=\Omega \mathscr{I}_{[a, z]} \quad \text { avec } \quad \Omega:=\sum_{\sigma \in \Gamma \cup\{0\}} \omega_{\sigma}(z) x_{\sigma} .
$$

La primitivité de $\Omega$ implique que $\Delta \mathscr{I}_{[a, z]}$ et $\mathscr{I}_{[a, z]} \otimes \mathscr{I}_{[a, z]}$ sont solutions de $d \mathscr{J}=$ $\Delta(\Omega) \mathscr{J}$, avec la même condition initiale $\mathscr{J}(a)=1 \otimes 1$.

Plus généralement, $\mathscr{I}_{\gamma}$ est le transport parallèle de 1 le long de $\gamma$ de la connexion intégrable $d-$ (multiplication à gauche par $\Omega$ ) sur le fibré trivial $\left(\mathbf{G}^{*} \backslash \Gamma\right) \times$ $\mathbf{C}\langle\langle\mathrm{X}\rangle\rangle$. C'est ainsi qu'est explicitée la comparaison Betti-de Rham pour $\pi_{1}\left(\mathbf{G}_{\mathrm{m}} \backslash \boldsymbol{\mu}_{n}\right)$ $[9,12.16]$. Pour $\Gamma=\mathbf{1}$, l'équation (2.2.4.1) est la réduction à $\mathbf{P}^{1} \backslash\{0,1, \infty\}$ du système de Knizhnik-Zamolodchikov $\mathrm{KZ}_{3}$ utilisée par Drinfel'd [13].

2.2.5. Convergence partielle. - Pour $(n, v) \in \mathbf{N}^{*} \times \Gamma$, soit $y_{n, v}=x_{0}^{n-1} x_{v}$. Notons $\mathrm{Y}_{\Gamma}$ l'ensemble des $y_{n, v}$, ou simplement $\mathrm{Y}$ s'il n'y a pas d'ambiguité sur $\Gamma$. La sous-algèbre de $\mathbf{Q}\langle\mathrm{X}\rangle$ engendrée par $\mathrm{Y}$ est libre sur Y. Comme espace vectoriel, elle est engendrée par les mots en $\mathrm{X}$ ne se terminant pas par $x_{0}$ et il sera pratique de l'identifier au quotient de $\mathbf{Q}\langle\mathrm{X}\rangle$ par l'idéal à droite homogène $\mathrm{I}_{0}=\mathbf{Q}\langle\mathrm{X}\rangle x_{0}$. On notera $\pi_{\mathrm{Y}}$ la projection correspondante.

Comme $x_{0}$ est primitif, l'idéal $\mathrm{I}_{0}$ est un coïdéal pour $\Delta$. Ceci fait du quotient $\mathbf{Q}\langle\mathrm{X}\rangle / \mathrm{I}_{0}$ une cogèbre graduée, qu'on notera par abus $(\mathbf{Q}\langle\mathrm{Y}\rangle, \Delta)$. Par homogénéité, ces constructions passent à l'extension des scalaires complétée. Ceci ne fait pas de $(\mathbf{Q}\langle\mathrm{Y}\rangle, \cdot, \Delta)$ une bigèbre : le coproduit-quotient $\Delta$ n'est plus un morphisme d'algèbres.

Considérons $\mathrm{I}_{[a, z]}$ comme une fonction de la variable $a$ de ]0, 1[. Par la proposition 2.2.4, L'élément $\pi_{\mathrm{Y}}\left(\mathscr{I}_{[a, z]}\right)$ de $\left(\mathbf{C}^{] 0,1[}\langle\langle\mathrm{Y}\rangle\rangle, \Delta\right)$ est diagonal pour $\Delta$, car $\pi_{\mathrm{Y}}$ est un morphisme de cogèbres. Ses coefficients, du type $\mathrm{I}_{[a, z]}\left(a_{1}, \ldots, a_{r}\right)$ avec $a_{r} \neq 0$, admettent une limite lorsque $a$ tend vers 0 dans ]0, 1[ (prop. 2.2.2). Le passage à la limite étant un morphisme d'algèbres, on en déduit :

Proposition 2.2.6. - Pour tout $z \in] 0,1[$, la série

$$
\mathscr{I}_{[0, z]}=\sum_{p \in \mathbf{N}, a_{1}, \ldots, a_{p-1} \in \Gamma \cup\{0\}, a_{p} \in \Gamma} \mathrm{I}_{[0, z]}\left(a_{1}, \ldots, a_{p}\right) x_{a_{1}} \cdots x_{a_{p}}
$$

est un élément diagonal de la cogèbre $(\mathbf{C}\langle\langle\mathrm{Y}\rangle\rangle, \Delta)$. 
DOUBLES MÉLANGES DES POLYLOGARITHMES MULTIPLES AUX RACINES DE L'UNITÉ 197

2.2.7. Tout mot $w$ en $\mathrm{X}$ s'écrit uniquement $x_{0}^{n_{1}} x_{\sigma_{1}} x_{0}^{n_{2}} x_{\sigma_{2}} \cdots x_{0}^{n_{r}} x_{\sigma_{r}} x_{0}^{n_{r+1}}$, les $n_{i}$ étant dans $\mathbf{N}$ et les $\sigma_{i}$ dans $\Gamma$. Soient $\mathbf{p}$ et $\mathbf{q}$ (produits et quotients successifs) les endomorphismes linéaires de $\mathbf{Q}\langle\mathrm{X}\rangle$, définis par :

$$
\mathbf{p}\left(x_{0}^{n_{1}} x_{\sigma_{1}} x_{0}^{n_{2}} x_{\sigma_{2}} \cdots x_{0}^{n_{r}} x_{\sigma_{r}} x_{0}^{n_{r+1}}\right)=x_{0}^{n_{1}} x_{\sigma_{1}} x_{0}^{n_{2}} x_{\sigma_{1} \sigma_{2}} \cdots x_{0}^{n_{r}} x_{\sigma_{1} \sigma_{2} \cdots \sigma_{r}} x_{0}^{n_{r+1}}
$$

$$
\mathbf{q}\left(x_{0}^{n_{1}} x_{\sigma_{1}} x_{0}^{n_{2}} x_{\sigma_{2}} \cdots x_{0}^{n_{r}} x_{\sigma_{r}} x_{0}^{n_{r+1}}\right)=x_{0}^{n_{1}} x_{\sigma_{1}} x_{0}^{n_{2}} x_{\sigma_{2} \sigma_{1}^{-1}} \cdots x_{0}^{n_{r}} x_{\sigma_{r} \sigma_{r-1}^{-1}} x_{0}^{n_{r+1}},
$$

Il est clair que $\mathbf{p}$ et $\mathbf{q}$ sont inverses l'un de l'autre et homogènes pour le poids, ce qui permet d'étendre leurs définitions à $k\langle\langle\mathrm{X}\rangle\rangle$, et que leurs restrictions à $k\langle\langle\mathrm{Y}\rangle\rangle$, qui est stable, sont données par :

$$
\mathbf{p}\left(y_{s_{1}, \sigma_{1}} y_{s_{2}, \sigma_{2}} \cdots y_{s_{r}, \sigma_{r}}\right)=y_{s_{1}, \sigma_{1}} y_{s_{2}, \sigma_{1} \sigma_{2}} \cdots y_{s_{r}, \sigma_{1} \sigma_{2} \cdots \sigma_{r}}
$$

$$
\mathbf{q}\left(y_{s_{1}, \sigma_{1}} y_{s_{2}, \sigma_{2}} \cdots y_{s_{r}, \sigma_{r}}\right)=y_{s_{1}, \sigma_{1}} y_{s_{2}, \sigma_{2} \sigma_{1}^{-1}} \cdots y_{s_{r}, \sigma_{r} \sigma_{r-1}^{-1}},
$$

pour tous entiers $s_{1}, \ldots, s_{r}$ strictement positifs et tous éléments $\sigma_{1}, \ldots, \sigma_{r}$ de $\Gamma$.

Considérons également la série génératrice

$$
\mathcal{L}_{z}:=\sum_{s_{1}, \ldots, s_{r}, \sigma_{1}, \ldots, \sigma_{r}} \mathrm{~L}_{s_{1}, \ldots, s_{r}}\left(z \sigma_{1}, \ldots, \sigma_{r}\right) y_{s_{1}, \sigma_{1}} \cdots y_{s_{1}, \sigma_{r}} .
$$

Avec ces notations, la proposition 2.2.2 se réécrit :

Proposition 2.2.8. - Pour tout $z \in] 0,1\left[\right.$, on a $\mathcal{L}_{z}=\mathbf{q}\left(\mathscr{I}_{z}\right)$ dans $\mathbf{C}\langle\langle\mathrm{Y}\rangle\rangle$

Ceci peut aussi s’interpréter comme égalité de deux éléments de $\mathbf{G}^{] 0,1[}\langle\langle\mathrm{Y}\rangle\rangle$.

2.2.9. Convergence totale. - Le sous-espace vectoriel $\mathbf{Q}\langle\mathrm{X}\rangle_{\mathrm{cv}}$ de $\mathbf{Q}\langle\mathrm{X}\rangle$ engendré par les mots en $\mathrm{X}$ ne se terminant pas par $x_{0}$ et ne commençant pas par $x_{1}$ est lui aussi une sous-algèbre graduée, qu'on identifiera au quotient $\mathbf{Q}\langle\mathrm{X}\rangle / \mathrm{I}$ avec $\mathrm{I}=\left(x_{1} \mathbf{Q}\langle\mathrm{X}\rangle+\right.$ $\left.\mathbf{Q}\langle\mathrm{X}\rangle x_{0}\right)$. On notera $\pi_{\mathrm{cv}}$ la projection correspondante. Par primitivité de $x_{0}$ et $x_{1}$, le sous-espace I est un coïdéal, d'où un coproduit-quotient, toujours noté $\Delta$ sur $\mathbf{Q}\langle\mathrm{X}\rangle_{\mathrm{cv}}$.

La proposition 2.2.8 montre que les coefficients de $\pi_{\mathrm{cv}}\left(\mathscr{I}_{z}\right)$ sont les fonctions associées comme en 2.1.2 aux suites $\left(\mathrm{L}_{s_{1}, \ldots, s_{r}}^{\mathrm{N}}\left(\sigma_{1}, \ldots, \sigma_{r}\right)\right)_{\mathrm{N}>0}$, qui sont convergentes (cor. 2.1.8). Le lemme d'Abel permet de répéter le même argument qu'en 2.2.5 :

Proposition 2.2.10. - La série $\mathscr{I}_{\mathrm{cv}}:=\lim _{a \rightarrow 0^{+}, b \rightarrow 1^{-}} \pi_{\mathrm{cv}}\left(\mathscr{I}_{a}^{b}\right)$ est un élément diagonal de $\left(\mathbf{C}\langle\langle\mathrm{X}\rangle\rangle_{\mathrm{cv}}, \Delta\right)$.

L'espace vectoriel $\mathbf{Q}\langle\mathrm{X}\rangle_{\mathrm{cv}}$ est également la sous-algèbre de $\mathbf{Q}\langle\mathrm{Y}\rangle$ engendrée par les mots en $\mathrm{Y}$ ne commençant pas par $y_{1,1}=x_{1}$. On l'identifie encore au quotient $\mathbf{Q}\langle\mathrm{Y}\rangle / y_{1,1} \mathbf{Q}\langle\mathrm{Y}\rangle$. Cela nous amènera à noter indifféremment $\mathbf{Q}\langle\mathrm{Y}\rangle_{\mathrm{cv}}$ pour $\mathbf{Q}\langle\mathrm{X}\rangle_{\mathrm{cv}}$. De plus, on voit facilement que $\mathbf{p}$ et $\mathbf{q}$ commutent à $\pi_{\mathrm{cv}}$. 
Proposition 2.2.11. - La série génératrice

$$
\text { (2.2.11.1) } \quad \mathcal{L}_{\mathrm{cv}}:=\sum_{s_{1}, \ldots, s_{r}, \sigma_{1}, \ldots, \sigma_{r},\left(s_{1}, \sigma_{1}\right) \neq(1,1)} \mathrm{L}_{s_{1}, \ldots, s_{r}}\left(\sigma_{1}, \ldots, \sigma_{r}\right) y_{s_{1}, \sigma_{1}} \cdots y_{s_{r}, \sigma_{r}}
$$

est égale à $\mathbf{q}\left(\mathscr{I}_{\mathrm{cv}}\right)$ dans $\mathbf{G}\langle\langle\mathrm{Y}\rangle\rangle_{\mathrm{cv}}$.

En effet, $\mathcal{L}_{\mathrm{cv}}$ et $\mathbf{q}\left(\mathscr{I}_{\mathrm{cv}}\right)$ sont les limites terme à terme de $\pi_{\mathrm{cv}}\left(\mathcal{L}_{z}\right)$ et $\mathbf{q}\left(\pi_{\mathrm{cv}}\left(\mathscr{I}_{[0, z]}\right)\right)$ lorsque $z$ tend vers $1^{-}$.

2.3. Relation de mélange des sommes itérées. - On se propose ici de décrire de manière compacte toutes les relations du type (1.1.5), lorsque les variables sont dans un sous-groupe multiplicatif fini $\Gamma$ fixé de $\mathbf{C}$. Comme en 2.2, cela se traduira par la diagonalité de certaines séries génératrices.

2.3.1. Définitions. - Dans $\mathbf{Q}\langle\mathrm{Y}\rangle$, on étend la notation $y_{n, v}$ au cas $n=0$ par la convention

$$
\text { (2.3.1.1) } \quad y_{0, \sigma}:=\left\{\begin{array}{lll}
1 & \text { si } & \sigma=1 \\
0 & \text { si } & \sigma \neq 1
\end{array}\right.
$$

L'algèbre $\mathbf{Q}\langle\mathrm{Y}\rangle$ admet une $(\mathbf{N} \times \Gamma)$-graduation, le poids coloré, obtenue en convenant que $y_{n, v}$ est de degré $(n, v)$. La première composante, le poids, est héritée de $\mathbf{Q}\langle\mathrm{X}\rangle$. La seconde est une $\Gamma$-graduation, qu'on appellera la couleur totale. En convenant que chaque élément de $\mathrm{Y}$ est de degré 1, on définit une autre $\mathbf{N}$-graduation, la longueur ${ }^{(3)}$ qui jouera un rôle moins important dans la suite. La différence du poids et de la longueur est le degré partiel en $x_{0}$ de $\mathbf{Q}\langle\mathrm{X}\rangle$.

Le coproduit $\Delta_{\star}$, défini comme morphisme d'algèbres par la condition

$$
\Delta_{\star}\left(y_{n, v}\right):=\sum_{\substack{k+l=n \\ \kappa \lambda=v}} y_{k, \kappa} \otimes y_{l, \lambda},
$$

est clairement cocommutatif, coassociatif et coünifère et fait de $\left(\mathbf{Q}\langle\mathrm{Y}\rangle, \cdot \cdot \Delta_{\star}\right)$ une bigèbre de Hopf - l'antipode est fourni par 2.3.6 ci-dessous. Il est de plus homogène pour le poids (et même pour le poids coloré), ce qui permet de le prolonger à $k\langle\langle\mathrm{Y}\rangle\rangle$. Par contre, il ne respecte pas la graduation de longueur, mais seulement la filtration décroissante associée.

Il est caractérisé, en tant que morphisme d'algèbres homogène pour le poids coloré, par le fait que l'élément

$$
\mathscr{Y}:=\sum_{(n, v) \in \mathbf{N} \times \Gamma} y_{n, v}
$$

de $\mathbf{Q}\langle\langle Y\rangle\rangle$ est diagonal, comme le montre un calcul immédiat.

(3) Goncharov utilise le terme de «profondeur» (depth). 
DOUBLES MÉLANGES DES POLYLOGARITHMES MULTIPLES AUX RACINES DE L'UNITÉ 199

2.3.2. Sommes partielles. — Les relations du type (1.1.5) sont déjà vraies pour les sommes partielles $\mathrm{L}_{s_{1}, \ldots, s_{r}}^{\mathrm{N}}\left(z_{1}, \ldots, z_{r}\right)$ définies en 2.1.7.

Fixons $\mathrm{N}>0$ et considérons dans $\mathbf{C}\langle\langle\mathrm{Y}\rangle\rangle$ la série génératrice

$$
\mathcal{L}_{\mathrm{N}}:=\sum_{r, s_{1}, \ldots, s_{r}, \sigma_{1}, \ldots, \sigma_{r}} \mathrm{~L}_{s_{1}, \ldots, s_{r}}^{\mathrm{N}}\left(\sigma_{1}, \ldots, \sigma_{r}\right) y_{s_{1}, \sigma_{1}} \ldots y_{s_{r}, \sigma_{r}} .
$$

Ne pas fixer $\mathrm{N}$ revient à la voir comme un élément de $\mathbf{C}^{\mathbf{N}}\langle\langle\mathrm{Y}\rangle\rangle$, plus précisément de $\operatorname{DivLog}_{\mathbf{N}}\langle\langle\mathrm{Y}\rangle\rangle$ (cor. 2.1.8).

Pour $\lambda \in \mathbf{C}$ et $v \in \Gamma$, les substitutions $y_{n, v} \mapsto \lambda^{n} y_{n, v}$ et $y_{n, v} \mapsto v y_{n, v}$ sont des morphismes de cogèbres : cela peut se voir par test sur les générateurs, ou comme conséquence de l'homogénéité de $\Delta_{\star}$ pour le poids coloré. En appliquant une fois la première et $m$ fois la seconde à $\mathscr{Y}$, on obtient donc que l'élément

$$
\mathscr{Y}_{m}(\lambda):=\sum_{n \geq 0, v \in \Gamma} v^{m} \lambda^{n} y_{n, v}
$$

de $\mathbf{G}\langle\langle\mathrm{Y}\rangle\rangle$ est encore diagonal pour $\Delta_{\star}$.

Dans le développement du produit

$$
\mathrm{P}:=\mathscr{Y}_{\mathrm{N}-1}\left(\frac{1}{\mathrm{~N}-1}\right) \mathscr{Y}_{\mathrm{N}-2}\left(\frac{1}{\mathrm{~N}-2}\right) \ldots \mathscr{Y}_{1}\left(\frac{1}{1}\right)
$$

les occurences du mot $w:=y_{s_{1}, \sigma_{1}} \cdots y_{s_{r}, \sigma_{r}}$ correspondent aux suites $\mathrm{N}>n_{1}>\cdots>n_{r}$ d'entiers, le facteur $y_{s_{i}, \sigma_{i}}$ de $w$ provenant du facteur $\mathscr{Y}_{n_{i}}\left(1 / n_{i}\right)$ de P. Le coefficient de $y_{s_{i}, \sigma_{i}}$ dans $\mathscr{Y}_{n_{i}}\left(1 / n_{i}\right)$ étant $\sigma_{i}^{n_{i}} n_{i}^{-s_{i}}$, l'occurence $\mathrm{N}>n_{1}>\ldots>n_{r}$ de $w$ dans $\mathrm{P}$ porte donc le coefficient $\sigma_{1}^{n_{1}} n_{1}^{-s_{1}} \cdots \sigma_{r}^{n_{r}} n_{r}^{-s_{r}}$. Le coefficient total de $w$ dans $\mathrm{P}$ s'obtenant en sommant les coefficients portés par ces occurences, on voit donc que $\mathrm{P}$ est égal à $\mathcal{L}_{\mathrm{N}}$. Comme chaque $\mathscr{Y}_{i}(1 / i)$ est diagonal, on a donc démontré :

Proposition 2.3.3. — La série génératrice des sommes partielles $\mathcal{L}_{\mathrm{N}}$ est diagonale dans $\left(\mathbf{C}\langle\langle Y\rangle\rangle, \Delta_{\star}\right)$.

2.3.4. Quotient de convergence. - La formule (2.3.1.2) exprime en particulier la primitivité de $y_{1,1}$ dans $\left(\mathbf{Q}\langle\mathrm{Y}\rangle, \Delta_{\star}\right)$. L'idéal à droite $\mathrm{I}=y_{1,1} \mathbf{Q}\langle\mathrm{Y}\rangle$ est donc un coïdéal pour $\Delta_{\star}$, le quotient $\mathbf{Q}\langle\mathrm{Y}\rangle_{\mathrm{cv}}$ hérite du coproduit $\Delta_{\star}$ et la projection $\pi_{\mathrm{cv}}: \mathbf{Q}\langle\mathrm{Y}\rangle \rightarrow$ $\mathbf{Q}\langle\mathrm{Y}\rangle_{\mathrm{cv}}$ est un morphisme de cogèbres. Comme en 2.2.5, le projeté $\pi_{\mathrm{cv}}\left(\mathcal{L}_{\mathrm{N}}\right)$ est diagonal dans $\left(\mathbf{C}^{\mathbf{N}}\langle\langle\mathrm{Y}\rangle\rangle_{\mathrm{cv}}, \Delta_{\star}\right)$ et on peut passer à la limite :

Proposition 2.3.5. - L'élément $\mathcal{L}_{\mathrm{cv}}:=\lim _{\mathrm{N} \rightarrow \infty} \pi_{\mathrm{cv}}\left(\mathcal{L}_{\mathrm{N}}\right)$ de $\mathbf{C}\langle\langle\mathrm{Y}\rangle\rangle_{\mathrm{cv}}$ est diagonal pour le coproduit $\Delta_{\star}$. 
2.3.6. Redressement $d u$ coproduit $\Delta_{\star}$. - Malgré la parenté apparente, au moins au niveau informel, des deux types de relations de mélange, $\left(\mathbf{Q}\langle\mathrm{Y}\rangle, \cdot \Delta_{\star} \Delta_{\star}\right)$ n'est pas la bigèbre enveloppante de $\mathfrak{L i b}_{\mathbf{Q}}(\mathrm{Y})$, mais elle peut s'y ramener, par un automorphisme d'algèbres.

Soit $u_{n, v}$ la partie homogène de poids coloré $(n, v)$ de $\log (\mathscr{Y})(c f .2 .3 .1)$ et U l'ensemble des $u_{n, v},(n, v) \in \mathbf{N} \times \Gamma$ (en particulier, ceci impose $u_{0, v}=0$, pour toute valeur de $v$ ). Comme $\mathscr{Y}$ est diagonal, son logarithme est primitif; $\Delta_{\star}$ étant homogène pour le poids coloré, les $u_{n, v}$ sont tous primitifs.

Par définition, on a

$$
\exp \left(\sum_{n \in \mathbf{N}, v \in \Gamma} u_{n, v}\right)=\sum_{n \in \mathbf{N}, v \in \Gamma} y_{n, v} .
$$

En observant la partie homogène de poids coloré $(n, v)$ et de longueur 1 de cette expression, on voit que la partie de longueur 1 de $u_{n, v}$ est $y_{n, v}$. On a donc

$$
u_{s_{1}, \sigma_{1}} u_{s_{2}, \sigma_{2}} \cdots u_{s_{r}, \sigma_{r}}=y_{s_{1}, \sigma_{1}} y_{s_{2}, \sigma_{2}} \cdots y_{s_{r}, \sigma_{r}}+(\text { termes de longueur }>r) \text {. }
$$

En d'autres termes, la restriction à la composante homogène de poids $n$ de la substitution $y_{n, v} \mapsto u_{n, v}$ est unipotente. Cela assure que cette substitution est un automorphisme d'algèbres de $\mathbf{Q}\langle\mathrm{Y}\rangle$. Compte-tenu de la primitivité des $u_{n, v}$, on a donc prouvé :

Proposition 2.3.7. - La $\mathbf{Q}$-algèbre associative $\mathbf{Q}\langle\mathrm{Y}\rangle$ est librement engendrée par $\mathrm{U}$. La bigèbre $\left(\mathbf{Q}\langle\mathrm{Y}\rangle, \cdot \Delta_{\star}\right)$ est isomorphe à $(\mathbf{Q}\langle\mathrm{U}\rangle, \cdot, \Delta)$, bigèbre enveloppante universelle de $\mathfrak{L i b}_{\mathbf{Q}}(\mathrm{U})$.

En particulier, il existe une unique dérivation de $\mathbf{Q}\langle\mathrm{Y}\rangle$ qui envoie $u_{s, \sigma}$ sur 1 et annule tous les autres $u_{n, v}$. Appelons «longueur en $\mathrm{U}$ » la graduation pour laquelle les éléments de $\mathrm{U}$ sont de degré 1.

Proposition 2.3.8. - Pour $\psi$ primitif dans $\left(k\langle\langle\mathrm{Y}\rangle\rangle, \Delta_{\star}\right)$ et $(s, \sigma) \in \mathbf{N}^{*} \times \Gamma$, on a

$$
\partial_{u_{s, \sigma}}(\psi)=\left(\psi \mid y_{s, \sigma}\right) \text {. }
$$

Démonstration. - D’après la proposition précédente, $\psi$ est une série de Lie en les $u_{n, v}$, ainsi donc que $\mathrm{R}:=\psi-\sum_{n, v}\left(\psi \mid y_{n, v}\right) u_{n, v}$. Grâce à (2.3.6.2), on voit d'abord que $\mathrm{R}$ est sans terme de longueur 1, donc sans terme de longueur 1 en $\mathrm{U}$. Il appartient donc à $\left[\mathfrak{L i b}_{k}(\mathrm{U}), \mathfrak{L} \mathfrak{i b}_{k}(\mathrm{U})\right]$, qui est annulé par $\partial_{u_{s, \sigma}}$ car 1 est central, d'où le résultat.

2.3.9. Remarques. - Dans le cas $\Gamma=\mathbf{1}$, la bigèbre $\left(\mathbf{Q}\langle\mathrm{Y}\rangle, \cdot, \Delta_{\star}\right)$ est celle des fonctions symétriques non-commutatives [17], vue dans la base des fonctions com- 
plètes. Sa duale graduée est celle des fonctions quasi-symétriques (Qsym, $\star \delta)$ [32]. Les propositions 2.3.3 et 2.3.5 se dualisent en interprétant $\mathrm{L}_{\mathrm{N}}$ (resp. L), après extension par linéarité, comme un morphisme d'algèbres de Qsym dans $\mathbf{G}$ (resp. Qsym ${ }^{\mathrm{cv}} \rightarrow \mathbf{C}$, où Qsym $^{\text {cv }}$ est une sous-algèbre de Qsym, engendrée comme $\mathbf{Q}$-espace vectoriel par les éléments (monomiales) de la base duale de l'ensemble des mots en Y indicés par $\left(s_{1}, \ldots, s_{r}\right)$ avec $s_{1} \neq 1$; elle est duale du quotient $\left(\mathbf{Q}\langle\mathrm{Y}\rangle_{\mathrm{cv}}, \Delta_{\star}\right)$. Pour effectuer des calculs explicites, il est parfois préférable de travailler avec Qsym.

Pour généraliser à $\Gamma$ quelconque, M. Bigotte [3] a introduit l'algèbre des «fonctions quasi-symétriques colorées». On laisse au lecteur le soin de vérifier qu'elle est duale de la cogèbre $\left(\mathbf{Q}\left\langle\mathrm{Y}_{\Gamma}\right\rangle, \Delta_{\star}\right)$.

La proposition 2.3.7 est une généralisation directe du cas particulier $\Gamma=\mathbf{1}$, classique pour les fonctions symétriques non-commutatives. On a des descriptions plus fines de ce changement de base, ainsi que de nombreuses variantes [17,32]. On pourrait encore généraliser en remplaçant $\mathbf{N}^{*} \times \Gamma$ par un magma associatif et commutatif $(\mathrm{M},+)$ quelconque, dans lequel tout élément aurait un nombre fini de décompositions en sommes de deux éléments. La convention (2.3.1.1) se formulerait en adjoignant un élément neutre à $\mathrm{M}$.

2.3.10. Nous aurons dans la suite besoin d'un calcul qui se fait habituellement dans Qsym. Appliqué à l'élément diagonal $\mathcal{L}_{\mathrm{cv}}$, il redonne l'exemple 1.1 .5 de l'introduction (voir 1.6 pour la notation).

Proposition 2.3.11. - Pour tous $v \in k\langle\langle\mathrm{Y}\rangle\rangle, y_{s, \sigma} \in \mathrm{Y}$ et $y_{t, \tau} \in \mathrm{Y}$, on $a$ :

$$
\left(\Delta_{\star} v \mid y_{s, \sigma} \otimes y_{t, \tau}\right)=\left(v \mid y_{s+t, \sigma \tau}+y_{s, \sigma} y_{t, \tau}+y_{t, \tau} y_{s, \sigma}\right) \text {. }
$$

Pour v primitif pour $\Delta_{\star}$, on a donc

$$
\left(v \mid y_{s+t, \sigma \tau}+y_{s, \sigma} y_{t, \tau}+y_{t, \tau} y_{s, \sigma}\right)=0 \text {. }
$$

Démonstration. - Comme $\Delta_{\star}$ respecte la filtration de longueur, seuls les termes de longueur 1 et 2 de $v$ interviennent. L'identification est alors immédiate. La conséquence (2.3.11.1) est évidente.

2.4. Régularisations. - On définit ici deux relevés particuliers, notés $\mathscr{I}$ et $\mathscr{L}$, de $\mathscr{I}_{\mathrm{cv}}$ et $\mathcal{L}_{\mathrm{cv}}$, dans les éléments diagonaux, respectivement, de $(\mathbf{G}\langle\langle\mathrm{X}\rangle\rangle, \Delta)$ et $\left(\mathbf{G}\langle\langle\mathrm{Y}\rangle\rangle, \Delta_{\star}\right)$.

On peut considérer les coefficients apparaissant respectivement dans $\mathscr{I}$ et $\mathscr{L}$ comme des valeurs régularisées des intégrales itérées et des sommes itérées préservant la relation de mélange adéquate. Il est bien connu que la formule de Kontsevitch ne survit pas au processus. Dans notre formalisme, cela s'écrit $\mathscr{L} \neq \mathbf{q} \pi_{\mathrm{Y}}(\mathscr{I})$.

Les deux types de développements asymptotiques de 2.1 fournissent d'autres relevés, à coefficients polynomiaux. Les propriétés de comparaison de 2.1.4 se tradui- 
sent, grâce à un bon contrôle de l'ambiguïté des relèvements, par la relation de régularisation, qui exprime $\mathscr{L}$ en fonction de $\mathscr{I}$. On évoque ensuite ses conséquences et son historique.

2.4.1. Cadre général. - Soient $\mathfrak{g}$ une $\mathbf{Q}$-algèbre de Lie graduée, $\mathfrak{g}_{1}$ et $\mathfrak{g}_{2}$ deux sous-algèbres de Lie de $\mathfrak{g}$, telles que $\mathfrak{g}=\mathfrak{g}_{1} \oplus \mathfrak{g}_{2}$, en tant qu'espaces vectoriels.

Le théorème de Poincaré-Birkhoff-Witt montre que l'application

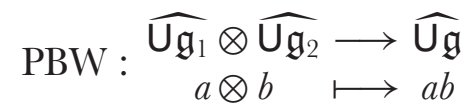

est un isomorphisme de cogèbres. Notons $\varepsilon$ la coünité de $\widehat{\mathrm{Ug}_{1}}$. L'application $\varepsilon \otimes \mathrm{Id}$ :

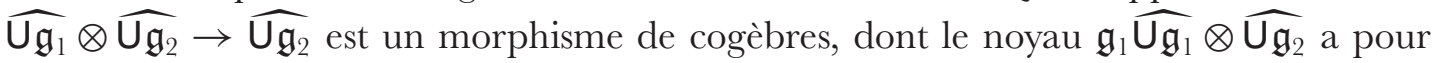
image $\mathfrak{g}_{1} \widehat{\cup \mathfrak{g}}$ par PBW. La cogèbre-quotient $\widehat{\mathrm{Ug}} / \mathfrak{g}_{1} \widehat{\cup \mathfrak{g}}$ est donc isomorphe à $\widehat{\mathrm{Ug}_{2}}$ et on a un diagramme commutatif de cogèbres :

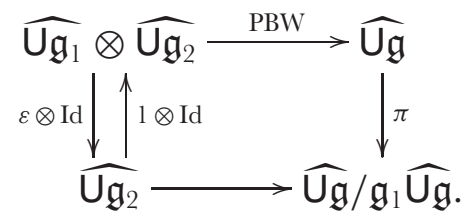

Les deux flèches horizontales de ce diagramme étant des isomorphismes de cogèbres, on déduit de $1 \otimes \mathrm{Id}$ une section de la projection canonique $\pi$, avec comme conséquence :

Proposition 2.4.2. - Tout élément diagonal de $\widehat{\mathrm{U}_{k} \mathfrak{g}} / \mathfrak{g}_{1} \widehat{\mathrm{U}_{k} \mathfrak{g}}$ est l'image par $\pi$ d'un élément de $\exp (k \mathfrak{g})$. Deux tels éléments se déduisent l'un de l'autre par multiplication à gauche par un élément de $\exp \left(k \mathfrak{g}_{1}\right)$.

On utilisera sans autre commentaire la variante pour les multiplications à droite.

2.4.3. Application à $\mathscr{I}_{\mathrm{cv}}$ et $\mathcal{L}_{\mathrm{cv}}$. - La proposition 2.4 .2 s'applique notamment lorsque $\mathfrak{g}$ est une algèbre de Lie libre sur un alphabet $\mathrm{A}$ et $\mathfrak{g}_{1}=\mathbf{Q} a$, pour $a \in \mathrm{A}$ : on prend pour $\mathfrak{g}_{2}$ le premier terme non trivial de la filtration décroissante associée au degré partiel en $\mathrm{A} \backslash\{a\}$.

La bigèbre enveloppante de $\mathfrak{L} \mathfrak{i b}(\mathrm{U})$ est $\left(\mathbf{Q}\langle\mathrm{Y}\rangle, \cdot \Delta_{\star}\right)$ (prop. 2.3.7) et on a $y_{1,1}=$ $u_{1,1} \in \mathrm{U}$. Comme les coefficients de $y_{1,1}$ s'additionnent lorsqu'on multiplie deux séries diagonales, on en déduit :

Corollaire 2.4.4. - Toute série $\Phi_{\mathrm{cv}}$ diagonale de $\left(\mathbf{Q}\langle\mathrm{Y}\rangle_{\mathrm{cv}}, \Delta_{\star}\right)$ est l'image par $\pi_{\mathrm{cv}}$ d'un élément diagonal de $\left(\mathbf{Q}\langle\mathrm{Y}\rangle, \Delta_{\star}\right)$. Deux tels éléments $\Phi_{1}$ et $\Phi_{2}$ sont liés par

$$
\Phi_{2}=\exp \left(\left(\lambda_{2}-\lambda_{1}\right) y_{1,1}\right) \Phi_{1},
$$

où $\lambda_{i}$ est le coefficient de $y_{1,1}$ dans $\Phi_{i}$, pour $i \in\{1,2\}$. 
DOUBLES MÉLANGES DES POLYLOGARITHMES MULTIPLES AUX RACINES DE L’UNITÉ 203

De même, on peut décomposer $\mathfrak{L} \mathfrak{i b}(\mathrm{X})$ en $\mathbf{Q} x_{1} \oplus \mathfrak{h} \oplus \mathbf{Q} x_{0}$ et appliquer deux fois la proposition 2.4.2. Il vient d'abord une variante pour $(\mathbf{Q}\langle\mathrm{Y}\rangle, \Delta)$ du corollaire 2.4.4, puis :

Corollaire 2.4.5. - Toute série $\Phi_{\mathrm{cv}}$ diagonale de $\left(\mathbf{Q}\langle\mathrm{X}\rangle_{\mathrm{cv}}, \Delta\right)$ est l'image par $\pi_{\mathrm{cv}}$ d'un élément diagonal de $(\mathbf{Q}\langle\mathrm{X}\rangle, \Delta)$. Deux tels éléments $\Phi_{1}$ et $\Phi_{2}$ sont liés par

$$
\Phi_{2}=\exp \left(\left(\lambda_{2}-\lambda_{1}\right) x_{1}\right) \Phi_{1} \exp \left(\left(\mu_{2}-\mu_{1}\right) x_{0}\right),
$$

où $\lambda_{i}$ et $\mu_{i}$ sont respectivement les coefficients de $x_{1}$ et $x_{0}$ dans $\Phi_{i}$, pour $i \in\{1,2\}$.

Ces corollaires peuvent également se déduire [23,33] d'un processus plus fin de décomposition des exponentielles de Lie, bien plus adapté pour les calculs informatiques que les développements en combinaisons linéaires de mots.

2.4.6. Notations. - On désignera par $\mathscr{I}$ l'unique série diagonale de $(\mathbf{C}\langle\langle\mathrm{X}\rangle\rangle, \Delta)$ telle que $\pi_{\mathrm{cv}}(\mathscr{I})=\mathscr{I}_{\mathrm{cv}}$ et dans laquelle les coefficients de $x_{0}$ et $x_{1}$ sont nuls. De même, l'unique élément diagonal de $\left(\mathbf{C}\langle\langle\mathrm{Y}\rangle\rangle, \Delta_{\star}\right)$ se projetant par $\pi_{\mathrm{cv}}$ sur $\mathcal{L}_{\mathrm{cv}}$ et dans laquelle le coefficient de $y_{1,1}$ est nul sera noté $\mathcal{L}$. L'existence et l'unicité de ces deux séries sont garanties par les corollaires 2.4.5 et 2.4.4.

La série $\pi_{\mathrm{Y}}(\mathscr{I})$ est caractérisée par le fait qu'elle est diagonale dans $(\mathbf{G}\langle\langle\mathrm{Y}\rangle\rangle, \Delta)$, antécédent de $\mathscr{I}_{\mathrm{cv}}$ par $\pi_{\mathrm{cv}}$ et que le coefficient de $y_{1,1}$ y est nul.

La suite de cette partie est consacrée à l'obtention d'une formule explicite de comparaison entre $\mathscr{I}$ et $\mathcal{L}$.

On adoptera la convention suivante : soient $k_{1}$ et $k_{2} \operatorname{deux} \mathbf{C}$-anneaux, $f: k_{1} \rightarrow k_{2}$ une application $\mathbf{C}$-linéaire et $\Phi \in k_{1}\langle\langle\mathrm{X}\rangle\rangle$. On note $\bar{f}(\Phi)$ l'élément de $k_{2}\langle\langle\mathrm{X}\rangle\rangle$ obtenu en appliquant $f$ aux coefficients de $\Phi$. L'application $\bar{f}$ ainsi définie est un morphisme de $\mathbf{G}\langle\langle\mathrm{X}\rangle\rangle$-bimodules. Si $f$ est un morphisme d'algèbres et $\Phi$ est diagonal pour $\Delta$, alors $\bar{f}(\Phi)$ l'est aussi. De même pour les $\mathrm{Y}$ et $\Delta_{\star}$.

2.4.7. Interprétation pour les sommes itérées. - Les coefficients de $\mathcal{L}_{\mathrm{N}}$ sont des suites de $\operatorname{DivLog}_{\mathbf{N}}$ (prop. 2.1.8). Comme As est un morphisme d'algèbres, $\mathcal{L}_{t}:=\overline{\mathrm{As}}\left(\mathcal{L}_{\mathrm{N}}\right)$ est diagonal dans $\left(\mathbf{C}[t]\langle\langle\mathrm{Y}\rangle\rangle, \Delta_{\star}\right)$; il se projette de plus par $\pi_{\mathrm{cv}}$ sur $\mathcal{L}_{\mathrm{cv}}$, si l'on considère $\mathbf{G}\langle\langle\mathrm{Y}\rangle\rangle$ comme inclus dans $\mathbf{G}[t]\langle\langle\mathrm{Y}\rangle\rangle$. Le coefficient de $y_{1,1}$ dans $\mathcal{L}_{t}$ étant $\operatorname{As}\left(\left(\mathrm{H}_{\mathrm{N}}\right)\right)$, égal à $\gamma+t$, le corollaire 2.4.4 appliqué avec $k=\mathbf{G}[t]$ détermine $\mathcal{L}_{t}$ :

$$
\mathcal{L}_{t}=\exp \left((\gamma+t) y_{1,1}\right) \mathcal{L} \text {. }
$$

2.4.8. Fustifications de 2.1.4. - Par $1^{k}$, on entend la séquence formée de $k$ fois le nombre 1. Par définition, $\mathrm{H}_{k, \mathrm{~N}}:=\mathrm{L}_{1^{k}}^{\mathrm{N}}\left(1^{k}\right)$ est le coefficient de $y_{1}^{k}$ dans $\mathcal{L}_{\mathrm{N}}$. Le polynôme $\operatorname{As}\left(\left(\mathrm{H}_{k, \mathrm{~N}}\right)\right)$ est donc le coefficient de $y_{1}^{k}$ dans $\mathcal{L}_{t}$. La formule (2.4.7.1) montre qu'il est de degré $k$ en $t$.

D'après la formule de Kontsevitch, $\mathrm{L}_{1^{k}}\left(1^{k-1}, z\right)$, valeur en $z$ de la fonction associée à $\mathrm{H}_{k, \mathrm{~N}}$, n'est autre que le coefficient de $x_{1}^{k}$ dans l'élement diagonal $\mathscr{I}_{[0, z]}$. D'après 
le lemme ci-dessous, c'est donc $\left(\mathrm{L}_{1}(z)\right)^{k} / k$ !, car le coefficient de $x_{1}$ dans $\mathscr{I}_{[0, z]}$ est $\mathrm{L}_{1}(z)$.

Lemme 2.4.9. - Soient $k$ un $\mathbf{Q}$-anneau, $\Phi$ un élément diagonal de $(k\langle\langle\mathrm{X}\rangle\rangle, \Delta)$ et $\lambda$ le coefficient de $x_{1}$ dans $\Phi$. Le coefficient de $x_{1}^{k}$ dans $\Phi$ est $\lambda^{k} / k$ !.

Démonstration. - Munie du coproduit $\Delta$ pour lequel $t$ est primitif, $\mathbf{Q} \llbracket t \rrbracket$ est la bigèbre enveloppante universelle complétée de l'algèbre de Lie de base $\{t\}$ et la substitution $\pi_{t}: x_{1} \mapsto t, x_{\alpha} \mapsto 0$ pour $\alpha \neq 1$ est un morphisme de cogèbres de $(k\langle\langle\mathrm{X}\rangle\rangle, \Delta)$ dans $(k \llbracket t \rrbracket, \Delta)$. Le coefficient de $x_{1}^{n}$ dans $\Phi$ est celui de $t^{n}$ dans $\pi_{t}(\Phi)$, qui, étant diagonal, est de la forme $\exp (\mu t)$.

2.4.10. Interprétation asymptotique pour les intégrales itérées. - Les coefficients de $\mathcal{L}_{z}$ et de $\mathscr{I}_{[0, z]}$ sont les fonctions $z \mapsto \mathrm{L}_{s_{1}, \ldots, s_{r}}\left(z \sigma_{1}, \ldots, \sigma_{r}\right)$, associées comme en 2.1.2 aux suites $\left(\mathrm{L}_{s_{1}, \ldots, s_{r}}^{\mathrm{N}}\left(\sigma_{1}, \ldots, \sigma_{r}\right)\right)_{\mathrm{N}>0}$, qui sont dans DivLog $\mathbf{N}_{\mathbf{N}}$. D'après le corollaire 2.1.5, dont on vient de compléter la démonstration, ce sont donc des éléments de DivLog $_{\mathrm{D}}$.

On peut donc poser $\mathscr{I}_{t}:=\overline{\mathrm{As}}\left(\mathscr{I}_{z}\right) ;$ c'est un élément diagonal de $(\mathbf{G}[t]\langle\langle\mathrm{Y}\rangle\rangle, \Delta)$ qui se projette par $\pi_{\mathrm{cv}}$ sur $\mathscr{I}_{\mathrm{cv}}$. Le coefficient de $x_{1}$ dans $\mathscr{I}_{t}$ vaut cette fois $t$, car c'est $\operatorname{As}\left(\mathrm{L}_{1}\right)$. Le corollaire 2.4.5 nous donne donc :

(2.4.10.1) $\quad \mathscr{I}_{t}=\exp \left(x_{1} t\right) \pi_{\mathrm{Y}}(\mathscr{I})$.

On voit au passage que $\pi_{\mathrm{Y}}(\mathscr{I})$ s'obtient — ainsi donc que les valeurs régularisées des intégrales itérées de 0 à $z$ - en prenant le terme constant des développements asympotiques polynomiaux en $\log (1-z)$ des intégrales itérées de 0 à $z$ au voisinage de $z=1$. On retrouve bien la «régularisation canonique» de Goncharov, et la description de l'image d'un chemin aboutissant à un point-base tangentiel dans la comparaison Betti-de Rham [9, 15.52].

2.4.11. Comparaison des deux régularisations. - On a $\mathcal{L}_{z}=\mathbf{q} \mathscr{I}_{[0, z]}$ et $\mathcal{L}_{z}$ se déduit de $\mathscr{L}_{\mathrm{N}}$ par l'application $\mathbf{C}$-linéaire $\operatorname{DivLog}_{\mathbf{N}} \rightarrow$ DivLog $_{\mathrm{D}}$ de 2.1.4.

D'après les définitions de $\mathscr{I}_{t}$ et $\mathcal{L}_{t}$ et le corollaire 2.1.5, on a $\overline{\operatorname{cmp}}\left(\mathscr{L}_{t}\right)=\mathbf{q} \mathscr{I}_{t}$. Comme $\overline{\mathrm{cmp}}$ est un endomorphisme de $\mathbf{C}\langle\langle\mathrm{Y}\rangle\rangle$-modules à droite, on déduit de (2.4.7.1) et $(2.4 .10 .1)$ que l'on a $\mathcal{L}=\mathscr{L}_{\text {corr }} \mathbf{q} \pi_{\mathrm{Z}}(\mathscr{I})$, avec $\mathcal{L}_{\text {corr }}:=\overline{\operatorname{cmp}}\left(\exp \left((\gamma+2 t) y_{1}\right)\right)$. La série $\mathcal{L}_{\text {corr }}$ est un produit d'éléments de $\mathbf{G}\langle\langle\mathrm{Y}\rangle\rangle$; elle est donc à coefficients dans $\mathbf{G}$. On a ainsi prouvé :

Proposition 2.4.12. - Il existe $\mathrm{S} \in \mathbf{C} \llbracket y_{1,1} \rrbracket$ telle que que

$$
\mathcal{L}=\mathrm{S} \cdot \mathbf{q} \pi_{\mathrm{Y}}(\mathscr{I}) .
$$


DOUBLES MÉLANGES DES POLYLOGARITHMES MULTIPLES AUX RACINES DE L’UNITÉ 205

2.4.13. Relation de régularisation. — La proposition 2.4.12 suffit à déterminer de manière purement algébrique les coefficients de $\mathrm{S}$.

Proposition 2.4.14. - Soient $k$ un $\mathbf{Q}$-anneau, $\Phi$ un élément diagonal de $(k\langle\langle\mathrm{X}\rangle\rangle, \Delta)$, sans terme en $x_{1}$, et une série $\mathrm{S}$ de $k \llbracket t \rrbracket$ telle que l'élément $\Phi_{\star}:=\mathrm{S}\left(y_{1,1}\right) \cdot \mathbf{q} \pi_{\mathrm{Y}}(\Phi)$ de $k\langle\langle\mathrm{Y}\rangle\rangle$ soit diagonal pour $\Delta_{\star}$. On a

$$
\mathrm{S}=\exp \left(\sum_{n \geq 1} \frac{(-1)^{n-1}}{n}\left(\Phi \mid x_{0}^{n-1} x_{1}\right) t^{n}\right)
$$

Démonstration. - L'application $\pi_{t}$ du lemme 2.4 .9 se factorise par $\pi_{\mathrm{Y}}$. Le quotient, noté encore $\pi_{t}$, est la substitution $y_{1,1} \mapsto t, y_{n, v} \mapsto 0$ pour $(n, v) \neq(1,1)$. Par le lemme 2.4.9, on a $\pi_{t} \mathbf{q} \pi_{\mathrm{Y}}(\Phi)=1$, d'où $\pi_{t}\left(\Phi_{\star}\right)=\mathrm{S}$.

D'après la proposition 2.3.7, on peut écrire $\Phi$ comme exponentielle d'une série de Lie en les $u_{n, v}$. Pour $n \neq 0$, notons $\alpha_{n, v}$ le coefficient de $u_{n, v}$ dans celle-ci. d'où

Comme $\mathbf{C} \llbracket t \rrbracket$ est commutative, tout crochet des $u_{n, v}$ est dans le noyau de $\pi_{t}$,

$$
\text { (2.4.14.1) } \quad \log \pi_{t}\left(\Phi_{\star}\right)=\sum_{n \in \mathbf{N}^{*}, v \in \Gamma} \alpha_{n, v} \pi_{t}\left(u_{n, v}\right) .
$$

On a $\pi_{t}\left(u_{n, v}\right)=0$ si $v \neq 1$, car $u_{n, v}$ est homogène de couleur totale $v$. On en déduit

$$
\sum_{n>0} \pi_{t}\left(u_{n, 1}\right)=\pi_{t}\left(\sum_{n \in \mathbf{N}, v \in \Gamma} u_{n, v}\right) \stackrel{\text { déf }}{=} \pi_{t} \log \left(\sum_{n \in \mathbf{N}, v \in \Gamma} y_{n, v}\right)=\log (1+t),
$$

d'où l'on tire $\pi_{t}\left(u_{n, 1}\right)=(-1)^{n-1} t^{n} / n$ par homogénéité de $\pi_{t}$. Reportons dans (2.4.14.1) :

$$
\text { (2.4.14.2) } \quad \log \pi_{t}\left(\Phi_{\star}\right)=\sum_{n>0} \frac{(-1)^{n-1}}{n} \alpha_{n, 1} t^{n} .
$$

Il reste à déterminer les $\alpha_{n, 1}$. À des termes de longueur au moins 2 près, on a $u_{n, v}=$ $y_{n, v}$ (cf. 2.3.6) et donc $\Phi_{\star}=\sum_{n, v} \alpha_{n, v} y_{n, v}$, car une série de Lie n'a pas de terme constant. On voit donc que $\alpha_{n, 1}$ est le coefficient de $y_{n, 1}$ dans $\Phi_{\star}$, i.e. $\left(\Phi \mid x_{0}^{n-1} x_{1}\right)$.

Le coefficient de $x_{0}^{n-1} x_{1}$ dans $\mathscr{I}$ vaut $\zeta(n)$ si $n \geq 2$ et 0 si $n=1$.

Corollaire 2.4.15 (Relation de régularisation). — Les séries $\mathcal{L}$ et $\mathscr{I}$ vérifient

$$
\mathcal{L}=\exp \left(\sum_{n \geq 2} \frac{(-1)^{n-1} \zeta(n)}{n} y_{1,1}^{n}\right) \mathbf{q} \pi_{\mathrm{Y}}(\mathscr{I})
$$


2.4.16. Conséquences. — Les relations DMR (doubles mélanges et régularisation) permettent de démontrer l'egalité $\zeta(2,1)=\zeta(3)$, qui était connue d'Euler, et ne peut se déduire des seuls doubles mélanges, relations de poids minimum 4.

Les relations DMR impliquent ${ }^{(4)}$ plus généralement la relation d'Hoffman [24], qui se généralise aux racines de l'unité [3] et sur laquelle les calculs de [23,3] se fondent, en plus des doubles mélanges : elle se ramène à l'absence de terme en $y_{1,1}$ dans le facteur correctif. On sait peu sur la réciproque, si ce n'est qu'elle est vraie pour les zêta multiples aussi loin que l'on puisse calculer par ordinateur avec les deux systèmes, i.e. jusqu'en poids 16 .

2.4.17. Remarques. - Le calcul de la proposition 2.4.14 se ramène à la variante de Waring des formules de Newton, modulo l'inclusion que nous n'avons pas détaillée de l'algèbre des fonctions symétriques dans celle des quasi-symétriques, elle-même incluse dans celle des fonctions quasi-symétriques colorées (cf. 2.3.9).

L'attribution de la relation de régularisation est difficile, même en faisant abstraction des différences de langage. Elle apparaît chez Goncharov [19,21], ce qui nous avait échappé à l'époque de la rédaction de [33], sous une forme duale de la proposition 2.4.12; chez Écalle [14], par des arguments très différents, modulo la correspondance entre ses moules entiers et nos séries génératrices, sous la forme 2.4.12 ; chez Boutet de Monvel [4], avec la définition et le calcul complet de cmp. Plus récemment, on trouve dans [25] des arguments proches, cette fois attribués à Zagier.

\subsection{Relations spécifiques aux racines de l'unité}

2.5.1. Les relations de distribution du polylogarithme classique se généralisent directement aux polylogarithmes multiples [21] :

Proposition 2.5.2. - Soient d un entier, $z_{1}, \ldots, z_{r}$ dans le disque unité fermé de $\mathbf{C}$. Si $\left(s_{1}, z_{1}\right) \neq(1,1)$, on $a$

$$
\sum_{t_{1}^{d}=z_{1}, t_{2}^{d}=z_{2}, \ldots, t_{r}^{d}=z_{r}} \mathrm{~L}_{s_{1}, \ldots, s_{r}}\left(t_{1}, \ldots, t_{r}\right)=d^{r-\left(s_{1}+\cdots+s_{r}\right)} \mathrm{L}_{s_{1}, \ldots, s_{r}}\left(z_{1}, \ldots, z_{r}\right) .
$$

Il s'agit de traduire cela en termes de séries génératrices non-commutatives, lorsque les $t_{i}$ appartiennent à $\Gamma \subset \mathbf{C}^{*}$ fixé.

2.5.3. Fonctorialités en $\Gamma$. — Tout morphisme de groupes $\varphi: \Gamma \rightarrow \Gamma^{\prime}$ donne lieu à deux substitutions $\varphi_{*}: \mathbf{Q}\left\langle\mathrm{X}_{\Gamma}\right\rangle \rightarrow \mathbf{Q}\left\langle\mathrm{X}_{\Gamma^{\prime}}\right\rangle$ et $\varphi^{*}: \mathbf{Q}\left\langle\mathrm{X}_{\Gamma^{\prime}}\right\rangle \rightarrow \mathbf{Q}\left\langle\mathrm{X}_{\Gamma}\right\rangle$, définies

(4) Voir [33] cor. III.4.21 p. 97 pour le détail, dans le cas des zêta multiples. 
par :

(2.5.3.1) $\quad \varphi^{*}\left(x_{\sigma}\right)=\left\{\begin{array}{lll}x_{0} & \text { si } \sigma=0 \\ \sum_{\tau \in \varphi^{-1}(\sigma)} x_{\tau} & \text { si } & \sigma \in \Gamma\end{array} \quad\right.$ et $\quad \varphi_{*}\left(x_{\sigma}\right)=\left\{\begin{array}{lll}d x_{0} & \text { si } & \sigma=0 \\ x_{\varphi(s)} & \text { si } & \sigma \neq 0,\end{array}\right.$

où $d$ désigne l'ordre de $\operatorname{ker} \varphi$.

Ces applications préservent les structures qu'on a précédemment définies. Ce sont évidemment des morphismes de cogèbres pour $\Delta$. Elles stabilisent les $\mathbf{Q}\langle Y\rangle$, commutent à $\pi_{\mathrm{Y}}$. Par test sur les $y_{n, v}$, on voit que ce sont des morphismes de cogèbres pour $\Delta_{\star}$. L'image directe $\varphi_{*}$ commute à $\mathbf{p}$ et $\mathbf{q}$; si $\varphi$ est injective, l'image réciproque $\varphi^{*}$ stabilise $\mathbf{Q}\langle\mathrm{Y}\rangle_{\mathrm{cv}}$ et commute à $\mathbf{p}$, $\mathbf{q}$ et $\pi_{\mathrm{cv}}$.

2.5.4. Soit $\Gamma$ un groupe commutatif fini. Pour tout diviseur $d$ de l'ordre de $\Gamma$, notons $p^{d}$ l'application $\sigma \mapsto \sigma^{d}$, restreinte à son image $\Gamma^{d}$ et $i_{d}$ l'inclusion de $\Gamma^{d}$ dans $\Gamma$.

Pour $\Gamma \subset \mathbf{G}^{*}$, on laisse au lecteur le soin de vérifier que la proposition 2.5.2 se traduit par l'égalité, dans $\mathbf{G}\left\langle\left\langle\mathrm{Y}_{\Gamma^{d}}\right\rangle\right\rangle$, de $i_{d}^{*}\left(\mathcal{L}_{\mathrm{cv}}\right)$ et $\pi_{\mathrm{cv}} p_{*}^{d}(\mathcal{L})$. La même égalité vaut pour $\mathscr{I}$, car $i_{d}^{*}$ et $p_{*}^{d}$ commutent à $\mathbf{p}$ et $\mathbf{q}$. Les séries $i_{d}^{*}(\mathscr{I})$ et $p_{*}^{d}(\mathscr{I})$, toutes deux diagonales, ont donc même image, $i_{d}^{*}\left(\mathcal{L}_{\mathrm{cv}}\right)$, par $\pi_{\mathrm{cv}}$. D'après le corollaire 2.4 .5 , il suffit de connaître leurs termes en $x_{0}$ et $x_{1}$ pour les comparer ; on en déduit facilement la relation de distribution régularisée :

Proposition 2.5.5. - Pour tout sous-groupe multiplicatif fini $\Gamma$ de $\mathbf{G}^{*}$ et tout diviseur $d$ de l'ordre de $\Gamma$, on $a$ :

$$
p_{*}^{d}(\mathscr{I})=\exp \left(\sum_{\sigma^{d}=1, \sigma \neq 1} \operatorname{Li}_{1}(\sigma) x_{1}\right) i_{d}^{*}(\mathscr{I}) .
$$

Par ailleurs, on a $\sum_{\sigma^{d}=1, \sigma \neq 1} \operatorname{Li}_{1}(\sigma)=-\log (d)$.

2.5.6. Relations de poids un. — Un calcul direct permet d'obtenir, pour $k$ strictement compris entre 0 et $n / 2$ :

$(2.5 .6 .1)$

$$
\left.\mathrm{L}_{1}(\exp (2 k i \pi / n))-\mathrm{L}_{1}(\exp (-2 k i \pi / n))\right)=(n-2 k) i \pi \text {. }
$$

Lorsque $v$ varie dans $\boldsymbol{\mu}_{n}(\mathbf{G})$, les $\mathrm{L}_{1}(v)-\mathrm{L}_{l}\left(v^{-1}\right)$ sont donc tous colinéaires sur $\mathbf{Q}$, avec des coefficients explicites, mais qui ne sont pas invariants par automorphismes de $\boldsymbol{\mu}_{n}(\mathbf{G})$, à l'exception de la conjugaison complexe. Si $n \notin\{1,2,3,4,6\}$, auquel cas $\boldsymbol{\mu}_{n}(\mathbf{G})$ a plus de deux automorphismes, on voit donc que les relations de poids un ne découlent pas des autres, qui le sont par 2.5.3.

D'après [11], ceci traduit la dépendance non fonctorielle de la correspondance Betti-de Rham du choix d'un plongement du corps cyclotomique dans C. Par contre, la dépendance par rapport au choix d'une clôture algébrique de $\mathbf{R}$ est fonctorielle, 
donnant lieu à l'invariance par conjugaison, qui a d'intéressantes conséquences (voir 5.3).

\section{3. Étude formelle des relations DMRD}

3.1. Le groupe MT. - On décrit ici, suivant la présentation de [11], le groupe par lequel se factorise l'action du groupe motivique $\mathscr{U}_{\mathrm{gr}}$ sur $\pi_{1}\left(\mathbf{G}_{\mathrm{m}} \backslash \boldsymbol{\mu}_{n}\right)$. Différentes variantes de ces formules apparaissent dans la littérature, provenant de la composition des automorphismes (extérieurs s'il y a des problèmes d'invariance de points-base) «spéciaux» de $\pi_{1}\left(\mathbf{G}_{\mathrm{m}} \backslash \boldsymbol{\mu}_{n}\right)$, équivariants pour certaines symétries.

3.1.1. Premières défnitions. - Soit $\Pi(k)$ la catégorie dont les objets sont $\mathrm{O}_{\sigma}$, pour $\sigma \in \Gamma \cup\{0\}$ et avec $\operatorname{Hom}_{\Pi(k)}\left(\mathrm{O}_{\sigma}, \mathrm{O}_{\tau}\right)=k\langle\langle\mathrm{X}\rangle\rangle$, la composition des flèches étant la multiplication des séries. Un élément $\mathrm{G}$ de $k\langle\langle\mathrm{X}\rangle\rangle$ sera noté $\mathrm{G}_{\tau, \sigma}$ lorsqu'il est vu dans $\operatorname{Hom}_{\Pi(k)}\left(\mathrm{O}_{\sigma}, \mathrm{O}_{\tau}\right)$.

Pour $\sigma \in \Gamma$, notons $t_{\sigma}$ la substitution $x_{v} \mapsto x_{\sigma v}$ de $k\langle\langle\mathrm{X}\rangle\rangle$. Soit $\mathrm{C}_{\sigma}$ l'endofoncteur de $\Pi(k)$ donné sur les objets par $\mathrm{O}_{v} \mapsto \mathrm{O}_{\sigma \nu}$ et sur les flèches par $t_{\sigma}$.

Dans le cas $\Gamma=\boldsymbol{\mu}_{n}$, la catégorie $\Pi(k)$ est la $k$-algèbre du groupoïde fondamental de $\mathbf{G}_{\mathrm{m}} \backslash \boldsymbol{\mu}_{n}$, restreint aux points-base tangentiels en 0 et $\boldsymbol{\mu}_{n}$, en réalisation «graduée ». Les $k$-points de ce dernier forment la sous-catégorie dont les flèches sont les exponentielles de Lie. Les $\mathrm{C}_{\sigma}$ proviennent de l'action de $\boldsymbol{\mu}_{n}$ sur $\mathbf{G}_{\mathrm{m}} \backslash \boldsymbol{\mu}_{n}$ par rotations.

Soit $\mathrm{E}(k)$ l'ensemble des endofoncteurs de $\Pi(k)$ agissant trivialement sur les objets, de manière $k$-linéaire et continue sur les flèches, qui fixent les $\left(x_{\sigma}\right)_{\sigma, \sigma}$ et qui commutent aux $\mathrm{G}_{\sigma}$. À $\mathscr{F} \in \mathrm{E}(k)$, faisons correspondre l'élément $\Phi(\mathscr{F})=\mathscr{F}\left(1_{1,0}\right)$. On définit ainsi une application de $\mathrm{E}(k)$ dans $k\langle\langle\mathrm{X}\rangle\rangle$.

3.1.2. Fixons $\mathscr{F} \in \mathrm{E}(k)$. Comme les flèches $1_{0,1}$ et $1_{1,0}$ sont inverses l'une de l'autre, $\Phi(\mathscr{F})$, on voit que $\mathscr{F}\left(1_{0,1}\right)$ est l'inverse de $\Phi(\mathscr{F})$. Pour $\sigma \in \Gamma$, de $\mathrm{C}_{\sigma}\left(1_{1,0}\right)=1_{\sigma, 0}$ découle $\mathscr{F}\left(1_{\sigma, 0}\right)=t_{\sigma}(\Phi(\mathscr{F}))$.

Soit $\kappa_{\mathrm{G}}$ la substitution $x_{\sigma} \mapsto\left[t_{\sigma}(\mathrm{G})\right]^{-1} x_{\sigma} t_{\sigma}(\mathrm{G}), x_{0} \mapsto x_{0}$. L'action de $\mathscr{F}$ sur $\operatorname{End}_{\Pi(k)}\left(x_{0,0}\right)$ est un morphisme continu de $k$-algèbres. De $\left(x_{\sigma}\right)_{0,0}=1_{0, \sigma}\left(x_{\sigma}\right)_{\sigma, \sigma} 1_{\sigma, 0}$ découle immédiatement que c'est $\kappa_{\Phi(\mathscr{F})}$.

Pour $\mathrm{G}, \mathrm{H} \in k\langle\langle\mathrm{X}\rangle\rangle$, posons, si $\mathrm{G}$ est inversible,

$$
\text { (3.1.2.1) } \quad \mathrm{G} \circledast \mathrm{H}:=\mathrm{G} \kappa_{\mathrm{G}}(\mathrm{H}) .
$$

En écrivant $\mathrm{H}_{1,0}=1_{1,0} \mathrm{H}_{0,0}$, on obtient $\mathscr{F}\left(\mathrm{H}_{1,0}\right)=\Phi(\mathscr{F}) \circledast \mathrm{H}$. Il est clair que l'application $\mathrm{G}$ est une bijection de $\mathrm{E}(k)$ sur l'ensemble des séries inversibles de $k\langle\langle\mathrm{X}\rangle\rangle$. Transportée par cette bijection, la composition de $\mathrm{E}(k)$ devient $\circledast$, en vertu de l'égalité tautologique de $\Phi\left(\mathscr{F}_{1} \mathscr{F}_{2}\right)$ et $\mathscr{F}_{1}\left(\Phi\left(\mathscr{F}_{2}\right)\right)$. 
DOUBLES MÉLANGES DES POLYLOGARITHMES MULTIPLES AUX RACINES DE L’UNITÉ 209

Le monoïde $\mathbf{E}$ est en fait un groupe. Pour cela, il suffit de vérifier la pleine fidélité de tout $\mathscr{F} \in \mathrm{E}(k)$, qu'on peut se contenter de tester sur $\operatorname{End}_{\Pi(k)}\left(\mathrm{O}_{0}\right)$. Or $\kappa_{\mathrm{G}}(\mathscr{F})$ est inversible, car pro-unipotent.

L'ensemble des $\mathscr{F}$ tels que le terme constant de $\Phi(\mathscr{F})$ soit 1 est clairement un sous-groupe de $\mathrm{E}(k)$. Dans ce cas, l'action sur $\operatorname{Hom}_{\Pi(k)}\left(\mathrm{O}_{0}, \mathrm{O}_{1}\right)$ est elle-même prounipotente. Elle est fidèle car $\mathscr{F}$ se déduit de $\mathscr{F}\left(1_{1,0}\right)$. Elle s'obtient par extension continue des scalaires de $\mathbf{Q}$ à $k$.

Proposition 3.1.3. - L'ensemble $\mathrm{MT}(k)$ des séries de terme constant 1, muni de la loi $\circledast$ définit un groupe pro-unipotent agissant par $\circledast$ sur $k\langle\langle\mathrm{X}\rangle\rangle$.

3.1.4. Remarques. - Par définition, l'action de $\mathrm{G} \in \mathrm{MT}(k)$ commute à la multiplication par gauche par $x_{1}$ et à droite par $x_{0}$ et passe donc aux quotients par $\pi_{\mathrm{Y}}$ et $\pi_{\mathrm{cv}}$.

Si $\mathrm{S} \in \mathrm{MT}(k)$ ne dépend que de $x_{1}$, l'automorphisme $\kappa_{\mathrm{S}}$ est l'identité, car $t_{\sigma}(\mathrm{S})$, ne dépendant que de $x_{\sigma}$, commute à $x_{\sigma}$. L'action de $\mathrm{S}$ est alors la multiplication à gauche par $\mathrm{S}$ et on a $\mathrm{G} \circledast \mathrm{S}=\mathrm{G} \circledast(\mathrm{S} \circledast 1)=\mathrm{SG}=\mathrm{S} \circledast \mathrm{G}$. Autrement dit, $\mathrm{S}$ est central.

3.1.5. Soit $\mathbf{F}(k)$ le sous-monoïde de $\mathbf{E}(k)$ agissant sur les flèches de $\Pi(k)$ par morphismes de cogèbres. L'élément $\Phi(\mathscr{F})$ paramétrant $\mathscr{F} \in \mathrm{F}(k)$ est diagonal, car $1_{1,0}$ l'est. Réciproquement, si $\mathrm{G}$ est un élément diagonal de $k\langle\langle\mathrm{X}\rangle\rangle$, le foncteur $\Phi^{-1}(\mathrm{G})$ appartient à $\mathrm{F}(k)$, car son action sur $\operatorname{Hom}_{\Pi(k)}\left(\mathrm{O}_{\sigma}, \mathrm{O}_{\tau}\right)$ est, comme le cas particulier (3.1.2.1), la composition d'une multiplication par un élément diagonal et d'opérateurs du type $\kappa_{\mathrm{G}}$, qui sont tous des morphismes de cogèbres, comme on le voit par test sur les éléments de X. En résumé :

Proposition 3.1.6. - $(\exp (\mathfrak{L i b}(\mathrm{X})), \circledast)$ est un sous-groupe pro-unipotent de $\mathrm{MT}$; il agit par morphisme de cogèbres sur $(k\langle\langle\mathrm{X}\rangle\rangle, \Delta)$.

On peut également considérer les sous-groupes de $\mathbf{E}$ qui respectent d'autres symétries, par exemple, pour $\Gamma=\mathbf{1}$, l'action de $\mathfrak{S}_{3}$ sur $\Pi(k)$ provenant de l'action sur $\mathbf{P}^{1} \backslash\{0,1, \infty\}$, ou, pour $\Gamma=\boldsymbol{\mu}_{n}$, l'action de $\mathbf{Z} / 2$ sur $\Pi(k)$ qui provient de l'inversion $\operatorname{sur} \mathbf{G}_{\mathrm{m}} \backslash \boldsymbol{\mu}_{n}$.

3.1.7. Explicitons les dépendances en $\Gamma$ par les notations $\Pi_{\Gamma}$ et $\mathrm{MT}(\Gamma)$. Un morphisme de groupes $\varphi: \Gamma \rightarrow \Gamma^{\prime}$ donne un foncteur $\Pi(\Gamma) \rightarrow \Pi\left(\Gamma^{\prime}\right)$, agissant sur les objets par $\mathrm{O}_{\sigma} \mapsto \mathrm{O}_{\varphi \sigma}$ et sur les flèches par $\varphi^{*}$. Il commute aux $\mathrm{C}_{\sigma}$. Ceci montre que $\varphi^{*}$ est un morphisme de groupes pro-unipotents $\operatorname{MT}(\Gamma) \rightarrow \operatorname{MT}\left(\Gamma^{\prime}\right)$, respectant les actions sur les $k\langle\langle\mathrm{X}\rangle\rangle$.

Si $\varphi$ est injectif, on définit un foncteur de la sous-catégorie pleine de $\Pi\left(\Gamma^{\prime}\right)$ d'objets les $\mathrm{O}_{\varphi(\sigma)}$ dans $\Pi(\Gamma)$ par $\mathrm{O}_{\varphi(\sigma)} \mapsto \mathrm{O}_{\sigma}$ et $\varphi_{*}$ sur les flèches et commutant aux $\mathrm{C}_{\sigma}$ 
appropriés. On en déduit que $\varphi_{*}$ est un morphisme $\operatorname{MT}\left(\Gamma^{\prime}\right) \rightarrow \operatorname{MT}(\Gamma)$, respectant les actions.

3.1.8. Structure infinitésimale. - L'algèbre de Lie $\mathfrak{m t}$ du groupe MT est formée des séries de terme constant nul. On notera dans la suite $\exp ^{\circledast}$ l'application exponentielle $\mathfrak{m t} \rightarrow \mathrm{MT}$, et $<_{\bullet}, \bullet>$ le crochet de $\mathrm{Lie}^{(5)}$ de $\mathfrak{m} \mathfrak{t}$, pour les distinguer de l'exponentielle et du crochet usuels de $k\langle\langle\mathrm{X}\rangle\rangle$. Le crochet de $\mathfrak{m t}$ sera appelé crochet d'Ihara. On peut expliciter cette structure grâce à la représentation dans $k\langle\langle\mathrm{X}\rangle\rangle$ :

Soit $\varepsilon$ une variable formelle telle que $\varepsilon^{2}=0$. Pour $\psi \in \mathfrak{m t}(k)$, notons $s_{\psi}$ l'opérateur qui associe à $v \in k\langle\langle\mathrm{X}\rangle\rangle$ le terme en $\varepsilon$ de $(1+\varepsilon \psi) \circledast v$. La machinerie standard sur les groupes pro-unipotents donne :

Proposition 3.1.9. - Pour tous $\psi \in \mathfrak{m} \mathfrak{t}(k)$ et $\mathrm{H} \in k\langle\langle\mathrm{X}\rangle\rangle$, on a

(3.1.9.1) $\quad \exp ^{\circledast}(\psi) \circledast \mathrm{H}=\exp \left(s_{\psi}\right)(\mathrm{H})$.

Pour tous $\psi_{1}, \psi_{2} \in \mathfrak{m t}(k)$, on a

(3.1.9.2) $\quad s_{<\psi_{1}, \psi_{2}>}=\left[s_{\psi_{1}}, s_{\psi_{2}}\right]$.

Tenant compte de $s_{\psi}(1)=\psi$ et $\mathrm{G} \circledast 1=\mathrm{G}$, on obtient en particulier :

Corollaire 3.1.10. - Pour tous $\psi_{1}, \psi_{2} \in \mathfrak{m t}(k)$, on a

(3.1.10.1) $\quad \exp ^{\circledast}(\psi)=\exp \left(s_{\psi}\right)(1)$

(3.1.10.2) $<\psi_{1}, \psi_{2}>=s_{\psi_{1}}\left(\psi_{2}\right)-s_{\psi_{2}}\left(\psi_{1}\right)$.

3.1.11. À partir des définitions, il est facile d'expliciter les opérateurs $s_{\psi}$ :

Proposition 3.1.12. - Pour tous $v \in k\langle\langle\mathrm{X}\rangle\rangle$ et $\psi \in \mathfrak{m t}(k)$, on a

(3.1.12.1) $\quad s_{\psi}(v)=\psi v+d_{\psi}(v)$,

où $d_{\psi}$ est la dérivation continue de $k\langle\langle\mathrm{X}\rangle\rangle$ caractérisée par :

$(3.1 .12 .2)$

$$
d_{\psi}\left(x_{\sigma}\right)=\left\{\begin{array}{lll}
{\left[x_{\sigma}, t_{\sigma}(\psi)\right]} & \text { si } & \sigma \neq 0 \\
0 & \text { si } & \sigma=0
\end{array}\right.
$$

On voit immédiatement que l'opération $\left(\psi_{1}, \psi_{2}\right) \mapsto d_{\psi_{1}}\left(\psi_{2}\right)$ est homogène pour le poids et la longueur (cf. 2.2.3), ainsi donc que $\left(\psi_{1}, \psi_{2}\right) \mapsto s_{\psi_{1}}\left(\psi_{2}\right)$. L'expression (3.1.10.2) du crochet d'Ihara met en évidence la structure d'algèbre pré-Lie de $\mathfrak{m t}$ (voir [18]).

(5) Cette notation suit celle de Drinfel'd [13, p. 851]. 
DOUBLES MÉLANGES DES POLYLOGARITHMES MULTIPLES AUX RACINES DE L’UNITÉ 211

\subsection{Résultat principal}

Définition 3.2.1. - Pour tout $\mathbf{Q}$-anneau $k$ et tout groupe commutatif fini $\Gamma$, on note $\underline{\operatorname{DMR}}(\Gamma)(k)$ l'ensemble des éléments $\Phi$ de $k\left\langle\left\langle\mathrm{X}_{\Gamma}\right\rangle\right.$ tels que :

$$
(\Phi \mid 1)=1 \text { et }\left(\Phi \mid x_{0}\right)=\left(\Phi \mid x_{1}\right)=0
$$

$$
\Delta \Phi=\Phi \widehat{\otimes} \Phi \text { et } \Delta_{\star} \Phi_{\star}=\Phi_{\star} \widehat{\otimes} \Phi_{\star} \text {, }
$$

où l'on pose $\Phi_{\star}:=\Phi_{\text {corr }} \cdot \mathbf{q} \pi_{\mathrm{Y}}(\Phi)$ et $\Phi_{\text {corr }}:=\exp \left(\sum_{n \geq 2} \frac{(-1)^{n-1}}{n}\left(\pi_{\mathrm{Y}}(\Phi) \mid y_{n, 1}\right) y_{1,1}^{n}\right)$.

Soit n l'ordre de $\Gamma$. On note $\underline{\operatorname{DMRD}}(\Gamma)(k)$ l'ensemble des éléments $\Phi$ de $\underline{\operatorname{DMR}}(\Gamma)(k)$ vérifiant pour tout diviseur $d$ de $n$ la relation de distribution :

$$
p_{*}^{d}(\Phi)=\exp \left(\sum_{\sigma^{d}=1}\left(\Phi \mid x_{\sigma}\right) x_{1}\right) i_{d}^{*}(\Phi) .
$$

Pour tout plongement ı de $\Gamma$ dans $\mathbf{G}^{*}$, on note $\operatorname{DMR}_{\lambda}^{\iota}(k)$ (resp. $\operatorname{DMRD}_{\lambda}^{\iota}(k)$ ) l'ensemble des éléments $\Phi$ de $\underline{\operatorname{DMR}}(\Gamma)(k)$ (resp. $\underline{\operatorname{DMRD}}(\Gamma)(k)$ ) vérifiant les relations de poids un :

$$
\left(\Phi \mid x_{\xi^{k}}-x_{\xi^{-k}}\right)=\frac{n-2 k}{n-2}\left(\Phi \mid x_{\xi}-x_{\xi^{-1}}\right),
$$

avec $\xi:=\iota^{-1}(\exp (2 i \pi / n))$ et $k$ compris entre 1 et $n / 2$, ainsi que la condition

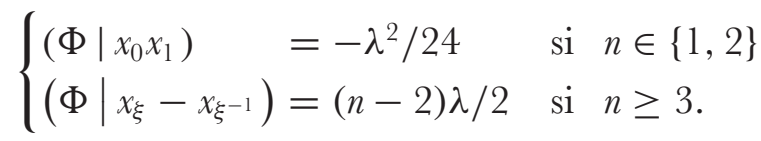

Soit enfin $\operatorname{DMR}^{\iota}(k)$ (resp. $\left.\operatorname{DMRD}^{\iota}(k)\right)$ l'ensemble des couples $(\lambda, \Phi)$, avec $\lambda \in k$ et $\Phi \in$ $\operatorname{DMR}_{\lambda}^{\iota}(k)\left(\right.$ resp. $\left.\Phi \in \operatorname{DMRD}_{\lambda}^{\iota}(k)\right)$.

3.2.2. Remarques. - La donnée de $\iota$ n'est bien sûr possible que si $\Gamma$ est cyclique, et équivaut à celle de $\xi$. Nombre de propriétés se formuleront néanmoins pour $\Gamma$ quelconque.

L'application $(\lambda, \Phi) \mapsto \lambda$ est un morphisme de schémas $\mathrm{DMR}^{\iota} \rightarrow \mathbf{A}^{1}$ et $\operatorname{DMR}_{\lambda}^{\iota}(k)$ est l'ensemble des $k$-points de la fibre de $\operatorname{DMR}^{\iota}$ au-dessus de $\lambda: \operatorname{Spec}(k)$ $\rightarrow \mathbf{A}^{1}$.

Dans le cas $n \leq 2$, le plongement $\iota$ est unique, la condition (3.2.1.4) est vide et les fibres $\mathrm{DMRD}_{\lambda}^{\iota}$ et $\mathrm{DMRD}_{-\lambda}^{\iota}$ sont égales.

Dans le cas $n>2$, on peut identifier $\operatorname{DMRD}^{l}(k)$ à l'ensemble des éléments de $\underline{\operatorname{DMRD}}(k)$ qui vérifient (3.2.1.4), car $\lambda$ se déduit de $\Phi$. Cela fait en particulier de $\operatorname{DMRD}_{0}^{\iota}(k)$ l'ensemble des $\Phi$ de $\underline{\operatorname{DMRD}}(k)$ qui vérifient $\left(\Phi \mid x_{\sigma}-x_{\sigma^{-1}}\right)=0$ pour tout $\sigma \in \Gamma$, et est donc indépendant de $\iota$. Les mêmes remarques s'appliquent aux DMR ${ }^{\iota}$. On fera parfois tomber $\iota$ de la notation dans ce cas. 
3.2.3. Le contenu de la section 2 se résume ainsi : pour tout $\iota$, la série $\iota^{*} \mathscr{I}(\iota(\Gamma))$, définie à partir des valeurs prises par les polylogarithmes multiples sur $\iota(\Gamma)$, est un élément de $\operatorname{DMRD}_{2 i \pi}^{\iota}(\mathbf{G})$, que l'on notera simplement $\mathscr{I}^{\iota}$. En effet, dans le cas $n \leq 2$, on a $\left(\mathscr{I} \mid x_{0} x_{1}\right)=\zeta(2)$, tandis que dans le cas $n \geq 3$, la condition (3.2.1.5) ne fait que traduire (2.5.6.1).

La preuve de la forme suivante de la propriété $(\mathbf{M})$ de l'introduction occupera quasiment toute la suite de cet article.

Théorème I. - Fixons un plongement ı d'un groupe commutatif fini $\Gamma$ dans $\mathbf{C}^{*}$.

- $\mathrm{DMR}_{0}^{\iota}$ et $\mathrm{DMRD}_{0}^{\iota}$ sont des sous-schémas en groupes de $\mathrm{MT}$.

- Pour tout $\mathbf{Q}$-anneau $k$ et $\lambda \in k$, les groupes $\operatorname{DMR}_{0}^{\iota}(k)$ et $\operatorname{DMRD}_{0}^{\iota}(k)$ agissent librement et transitivement par multiplication $\circledast$ à gauche respectivement sur $\operatorname{DMR}_{\lambda}^{\iota}(k)$ et $\operatorname{DMRD}_{\lambda}^{\iota}(k)$.

$-\operatorname{DMR}_{\lambda}^{\iota}(k)$ et $\operatorname{DMRD}_{\lambda}^{\iota}(k)$ sont non-vides.

3.3. Espaces tangents à l'origine. - La première étape de la preuve du théorème I concerne les espaces tangents au voisinage de 1 de $\underline{\mathrm{DMR}}$ et $\underline{\mathrm{DMRD}}$, i.e. les points de la forme $1+\varepsilon \psi$ à coefficients l'anneau des nombres duaux $k[\varepsilon]$.

On aboutit à la proposition 3.3.3, qui donne les contraintes portant sur les termes de longueur 1 de leurs éléments. Ce résultat explique le rôle de $2 i \pi$ dans le système DMRD.

3.3.1. Définitions. - Pour tout $\mathbf{Q}$-anneau $k$, et $\Gamma$ quelconque, soit $\mathfrak{d m r}(k)$ l'ensemble des séries $\psi$ de $k\langle\langle\mathrm{X}\rangle\rangle$ qui vérifient les équations :

$$
\left(\psi \mid x_{0}\right)=\left(\psi \mid x_{1}\right)=0
$$

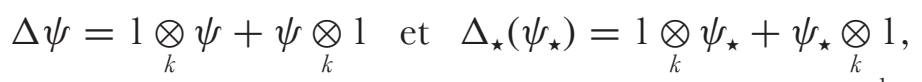

où l'on pose $\quad \psi_{\star}:=\mathbf{q} \pi_{\mathrm{Y}}(\psi)+\psi_{\text {corr }} \quad$ et $\quad \psi_{\text {corr }}:=\sum_{n \geq 2} \frac{(-1)^{n-1}}{n}\left(\psi \mid y_{n, 1}\right) y_{1,1}^{n}$.

Soit $\mathfrak{d} \mathfrak{m r d}(k)$ l'ensemble de celles qui vérifient en outre, pour tout diviseur $d$ de l'ordre de $\Gamma$, les relations de distribution

$$
p_{*}^{d}(\psi)=i_{d}^{*}(\psi)+\sum_{\sigma^{n / d}=1}\left(\psi \mid x_{\sigma}\right) x_{1} .
$$

3.3.2. On laisse le lecteur se convaincre du fait que $\mathfrak{d} \mathfrak{m r}$ et $\mathfrak{d} \mathfrak{m r d}$ sont, au sens plus haut, les espaces tangents à DMR et DMRD au voisinage de 1 .

Il est clair que la composante homogène de poids $p$ d'un élément de $\mathfrak{d} \mathfrak{m r}(k)$ est encore dans $\mathfrak{d} \mathfrak{m} \mathfrak{r}(k)$. On a donc là un schéma vectoriel ( $c f$. 1.6), associé au Qespace vectoriel gradué, qu'on notera encore $\mathfrak{d} \mathfrak{m} \mathfrak{r}$, des éléments de $\mathbf{Q}\langle\mathrm{X}\rangle$ qui sont dans $\mathfrak{d} \mathfrak{m} \mathfrak{r}(\mathbf{Q})$. De même pour $\mathfrak{d} \mathfrak{m} \mathfrak{r} \mathfrak{d}$. 
DOUBLES MÉLANGES DES POLYLOGARITHMES MULTIPLES AUX RACINES DE L’UNITÉ 213

Proposition 3.3.3. - Soient $\psi \in \mathfrak{d} \mathfrak{m r}, v \in \Gamma$ et $n$ un entier $\geq 2$. On a les égalités :

$$
\left(\psi_{\star} \mid y_{n, v}\right)+(-1)^{n}\left(\psi_{\star} \mid y_{n, v^{-1}}\right)=0 \text { si } n \geq 3 \text {; }
$$

$$
\left(\psi_{\star} \mid y_{n, v}\right)+\left(\psi_{\star} \mid y_{n, v^{-1}}\right)+\left(\psi_{\star} \mid y_{2,1}\right)=0 \text { si } n=2 \text { et } v \neq 1
$$

Lemme 3.3.4. - Pour tout élément $\psi$ de $\mathfrak{L i b}(\mathrm{X})$, tout $n \in \mathbf{N}^{*}$ et tout $v \in \Gamma$, on a

$$
\left(\mathbf{q} \pi_{\mathrm{Y}}(\psi) \mid y_{1,1} y_{n-1, v}+(-1)^{n} y_{1, v} y_{n-1, v^{-1}}\right)=0 \text {. }
$$

Démonstration. - De manière équivalente, on doit prouver que l'on a :

$$
\left(\psi \mid x_{1} x_{0}^{n-2} x_{v}+(-1)^{n} x_{v} x_{0}^{n-2} x_{1}\right)=0 .
$$

Comme $\psi \in \mathfrak{L} \mathfrak{i b}(\mathrm{X})$, l'antipode $\mathrm{S}_{\mathrm{X}}$ de la bigèbre enveloppante $\mathbf{Q}\langle\mathrm{X}\rangle$ le transforme en son opposé. Le coefficient de $x_{1} x_{0}^{n-2} x_{v}$ est donc l'opposé de celui de $\mathrm{S}_{\mathrm{X}}\left(x_{1} x_{0}^{n-2} x_{v}\right)=$ $(-1)^{n} x_{\nu} x_{0}^{n-2} x_{1}$.

Lemme 3.3.5. - Soient $n \geq 2$ un entier, $\sigma, \tau \in \Gamma$. Si $n \geq 3$ ou $(\sigma, \tau) \neq(1,1)$, on a, pour tout $\psi \in \mathfrak{L i b}(\mathrm{X})$ :

$$
\left(\psi_{\star} \mid y_{n-1, \tau} y_{1, \sigma \tau^{-1}}\right)+\sum_{p=1}^{n-1}\left(\psi_{\star} \mid y_{p, \sigma} y_{n-p, \tau \sigma^{-1}}\right)=0 .
$$

Démonstration. - On peut supposer $\psi$ homogène de poids $n$. Le terme correctif $\psi_{\text {corr }}$ est dans ce cas un multiple scalaire de $y_{1,1}^{n}$. L'hypothèse sur $n, \sigma$ et $\tau$ assure donc que les coefficients de $y_{1, \sigma} y_{1, \tau}$ dans $\mathbf{q} \pi_{\mathrm{Y}}(\psi)$ et $\psi_{\star}$ sont égaux. En utilisant $\mathbf{p}$, on est donc ramené à prouver :

$$
\left(\psi \mid x_{0}^{n-2} x_{\tau} x_{\sigma}\right)+\sum_{p=1}^{n-1}\left(\psi \mid x_{0}^{p-1} x_{\sigma} x_{0}^{n-p-1} x_{\tau}\right)=0 .
$$

Il s'agit en fait d'un calcul avec le produit $\sqcup$, dual de $\Delta$, dont on a jusqu'à présent évité l'emploi (voir [37]). Il est défini par $(a \mid b \sqcup c)=(\Delta(a) \mid b \otimes c)$ pour tous $a, b, c \in \mathbf{Q}\langle\mathrm{X}\rangle$. Le développement de $x_{\sigma} \sqcup x_{0}^{n-2} x_{\tau}$ est la somme de toutes les insertions possibles de $x_{\sigma}$ dans $x_{0}^{n-2} x_{\tau}$ :

$$
x_{\sigma} \sqcup x_{0}^{n-2} x_{\tau}=x_{0}^{n-2} x_{\tau} x_{\sigma}+\sum_{p=1}^{n-1} x_{0}^{p-1} x_{\sigma} x_{0}^{n-p-1} x_{\tau} .
$$

Comme $\psi$ est primitif, on a $\left(\psi \mid x_{\sigma} \sqcup x_{0}^{n-1} x_{\tau}\right)=\left(\Delta \psi \mid x_{\sigma} \otimes x_{0}^{n-1} x_{\tau}\right)=0$.

3.3.6. Démonstration de la proposition 3.3.3. — Le lemme 3.3.5, appliqué, pour $n \geq 3$ ou $v \neq 1$, à $(\sigma, \tau)=(\nu, v)$, puis $(1, v)$ donne : 
(3.3.6.1)

$$
\begin{aligned}
& \left(\psi_{\star} \mid y_{n-1, v} y_{1,1}\right)+\sum_{p=1}^{n-1}\left(\psi_{\star} \mid y_{p, v} y_{n-p, 1}\right)=0 \quad \text { et } \\
& \left(\psi_{\star} \mid y_{n-1, v} y_{1, v^{-1}}\right)+\sum_{p=1}^{n-1}\left(\psi_{\star} \mid y_{p, 1} y_{n-p, v}\right)=0, \quad \text { i.e. }
\end{aligned}
$$

$(3.3 .6 .2)$

$$
\left(\psi_{\star} \mid y_{n-1, \nu} y_{1, v^{-1}}\right)+\sum_{p=1}^{n-1}\left(\psi_{\star} \mid y_{n-p, 1} y_{p, v}\right)=0 \text {. }
$$

Or, pour tout $p$ compris entre 1 et $n-1$, la primitivité de $\psi_{\star}$ pour $\Delta_{\star}$ donne $(c f .2 .3 .11)$ :

$$
\left(\psi_{\star} \mid y_{n, v}+y_{p, v} y_{n-p, 1}+y_{n-p, 1} y_{p, v}\right)=0 \text {. }
$$

On tire donc en sommant les égalités (3.3.6.1) et (3.3.6.2) :

$(3.3 .6 .3)$

$$
\left(\psi_{\star} \mid y_{n-1, v} y_{1,1}+y_{n-1, v} y_{1, v^{-1}}\right)-(n-1)\left(\psi_{\star} \mid y_{n, v}\right)=0, \text { puis }
$$

$$
-\left(\psi_{\star} \mid y_{1,1} y_{n-1, v}+y_{1, v^{-1}} y_{n-1, v}\right)-n\left(\psi_{\star} \mid y_{n, v}\right)-\left(\psi_{\star} \mid y_{n, 1}\right)=0 .
$$

L'équation (3.3.6.4) sommée avec sa variante pour $v^{-1}$ pondérée par $(-1)^{n}$ donne :

$$
\begin{aligned}
& n\left(\psi_{\star} \mid y_{n, \nu}+(-1)^{n} y_{n, v^{-1}}\right)+\left(1+(-1)^{n}\right)\left(\psi_{\star} \mid y_{n, 1}\right)= \\
& \left(\psi_{\star} \mid y_{1,1} y_{n-1, \nu}+(-1)^{n} y_{1, v} y_{n-1, v^{-1}}\right)+\left(\psi_{\star} \mid y_{1, v^{-1}} y_{n-1, v}+(-1)^{n} y_{1,1} y_{n-1, v^{-1}}\right) .
\end{aligned}
$$

D’après le lemme (appliqué pour $v$ et $v^{-1}$ ), on a donc

$$
n\left(\psi_{\star} \mid y_{n, v}+(-1)^{n} y_{n, v^{-1}}\right)+\left(1+(-1)^{n}\right)\left(\psi_{\star} \mid y_{n, 1}\right)=0
$$

Pour $n$ impair et supérieur à 3 ou $n=2, v \neq 1$, l'équation (3.3.6.6) donne directement le résultat voulu. Pour $n$ pair, supérieur à 3 et $v=1$, l'équation (3.3.6.6) devient $\left(\psi_{\star} \mid y_{n, 1}\right)=0$, ce qui reporté dans (3.3.6.6) donne à nouveau (3.3.3.1).

On a une réduction supplémentaire :

Proposition 3.3.7. - Soit $\psi \in \mathfrak{d} \mathfrak{m r}$. Si $\Gamma$ est de cardinal au moins 3, le coefficient de $y_{2,1}$ dans $\psi$ est nul.

Démonstration. - Pour tout $\psi \in \mathbf{Q}\langle\mathrm{X}\rangle$, primitif pour $\Delta$, le coefficient de $x_{\sigma} \otimes x_{\sigma \tau}$ dans $\Delta \psi$ étant nul, on a $\left(\psi \mid x_{\sigma} x_{\sigma \tau}+x_{\sigma \tau} x_{\sigma}\right)=0$. Si $(\sigma, \tau) \neq(1,1)$, on en déduit la nullité de $\left(\mathbf{p} \psi_{\star} \mid x_{\sigma} x_{\sigma \tau}+x_{\sigma \tau} x_{\sigma}\right)$, ce qui donne :

$$
\left(\psi_{\star} \mid y_{1, \sigma} y_{1, \tau}\right)=-\left(\psi_{\star} \mid y_{1, \sigma \tau} y_{1, \tau^{-1}}\right)=\left(\psi_{\star} \mid y_{2, \sigma}+y_{1, \tau^{-1}} y_{1, \sigma \tau}\right),
$$


DOUBLES MÉLANGES DES POLYLOGARITHMES MULTIPLES AUX RACINES DE L’UNITÉ 215

la deuxième égalité provenant de la proposition 2.3.11. L'application $(\sigma, \tau) \mapsto$ $\left(\tau^{-1}, \sigma \tau\right)$ est cyclique d'ordre 6 . En itérant six fois l'égalité ci-dessus, on obtient facilement

$$
\left(\psi_{\star} \mid y_{2, \sigma}+y_{2, \tau^{-1}}+y_{2, \sigma^{-1} \tau^{-1}}+y_{2, \sigma^{-1}}+y_{2, \tau}+y_{2, \sigma \tau}\right)=0 .
$$

Grâce à la proposition 3.3.3, ceci entraîne la nullité de $\left(\psi_{\star} \mid y_{2,1}\right)$, à condition qu'il existe $\sigma$ et $\tau$ dans $\Gamma$ tels que $\sigma, \tau$ et $\sigma \tau$ soient tous différents de 1 .

Un groupe $\Gamma$ non trivial dans lequel un tel choix est impossible est de cardinal 2 : fixons en effet $\sigma \in \Gamma \backslash\{1\}$; tout élément $\tau$ de $\Gamma \backslash\{1\}$ est l'inverse de ce $\sigma$.

3.3.8. Définitions de $\mathfrak{d} \mathfrak{m} \mathfrak{r}_{0}$ et $\mathfrak{d} \mathfrak{m r} \mathfrak{d}_{0}$. — L'équation (3.3.3.1) interviendra à plusieurs reprises comme condition technique dans la suite. C'est cette condition qui est à l'origine du fait qu'on obtienne une action fibre à fibre de $\mathrm{DMR}_{0}$ sur $\mathrm{DMR}^{\iota}$, et non une structure de groupe sur $\mathrm{DMR}^{\iota}$.

On notera dans la suite $\mathfrak{d} \mathfrak{m} \mathfrak{r}_{0}$ et $\mathfrak{d} \mathfrak{m} \mathfrak{r} \mathfrak{d}_{0}$ l'ensemble des éléments $\psi$ de $\mathfrak{d} \mathfrak{m r}$ (resp. $\mathfrak{d} \mathfrak{m r d})$ qui vérifient (3.3.3.1) pour tout $(n, v) \in \mathbf{N}^{*} \times \Gamma$. Par les propositions 3.3.3 et 3.3.7, il suffit d'imposer (3.3.3.1) pour $n=1$ si $|\Gamma| \geq 3$; pour $(n, v)=(2,1)$ si $|\Gamma| \leq 2$.

Pour tout plongement $\iota$ de $\Gamma$ dans $\mathbf{C}^{*}$, on voit que $\mathfrak{d} \mathfrak{m} \mathfrak{r}_{0}$ est l'espace tangent à $\mathrm{DMR}_{0}^{\iota}$ au voisinage de 1 , indépendant de $\iota$.

De (3.3.3.1) découle qu'un élément $\psi$ de $\mathfrak{d} \mathfrak{m} \mathfrak{r}_{0}$ n'a pas de terme en $y_{n, 1}$ si $n$ est pair. Son terme correctif $\psi_{\text {corr }}$ est donc une série impaire en $y_{1}$.

\section{Action tangente}

Cette section est consacrée à la première étape de la preuve du théorème I, sous la forme qui suit :

Proposition 4.A. - Soient $\Gamma$ un groupe commutatif fini et $k$ un $\mathbf{Q}$-anneau.

i) Les espaces tangents $\mathfrak{d} \mathfrak{m} \mathfrak{r}_{0}$ et $\mathfrak{d} \mathfrak{m r d} \mathfrak{d}_{0}$ sont des sous-algèbres de Lie de $\mathfrak{m t}$.

ii) Les ensembles $\underline{\operatorname{DMR}}(k)$ et $\underline{\operatorname{DMRD}}(k)$ sont stables par $\exp \left(s_{\psi}\right)$, pour tout $\psi$ appartenant respectivement à $\mathfrak{d} \mathfrak{m} \mathfrak{r}_{0}(k)$ et $\mathfrak{d} \mathfrak{m r d} \mathfrak{d}_{0}(k)$.

iii) Soit $\iota$ un plongement de $\Gamma$ dans $\mathbf{G}^{*}$. L'action de $\exp \left(s_{\psi}\right)$ sur $\underline{\operatorname{DMR}}(k)$ laisse les $\operatorname{DMR}_{\lambda}^{\iota}(k)$ stables.

Grâce à la formule de Campbell-Hausdorff, le premier point implique que $\exp ^{\circledast}\left(\mathfrak{d} \mathfrak{m} \mathfrak{r}_{0}\right)$ et $\exp ^{\circledast}\left(\mathfrak{d} \mathfrak{m r d} \mathfrak{d}_{0}\right)$ sont des sous-schémas en groupes de MT. Le deuxième indique alors qu'il agit par multiplication $\circledast$ à gauche sur DMR (resp. DMRD).

De 4.1 à 4.4, on ne considère comme coproduit que $\Delta_{\star}$. Par "primitif», il faut donc entendre par exemple "primitif pour $\Delta_{\star}$ ». 
4.1. Les opérateurs infinitésimaux de $\mathrm{MT}$, vus $\operatorname{sur} \mathbf{Q}\langle\langle\mathrm{Y}\rangle\rangle$

4.1.1. Définitions. - Pour tous $\psi, v \in \mathbf{Q}\langle\langle\mathbf{X}\rangle\rangle$, comme $d_{\psi}$ est une dérivation qui annule $x_{0}$, on a

$$
s_{\psi}\left(v x_{0}\right)=\psi v x_{0}+d_{\psi}\left(v x_{0}\right)=\psi v x_{0}+d_{\psi}(v) x_{0} .
$$

Il s'ensuit que le noyau $\mathbf{Q}\langle\langle\mathrm{X}\rangle\rangle x_{0}$ de $\pi_{\mathrm{Y}}$ est stable par $s_{\psi}$. Soit alors $s_{\psi}^{\mathrm{Y}}$ l'endomorphisme linéaire de $\mathbf{Q}\langle\langle\mathrm{Y}\rangle\rangle$ tel que le diagramme ci-dessous commute :

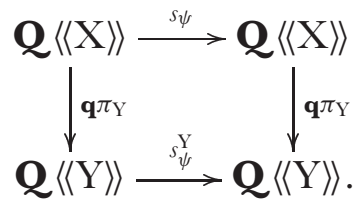

On considèrera également l'endomorphisme $\mathrm{D}_{\psi}^{\mathrm{Y}}$ de $\mathbf{Q}\langle\langle\mathrm{Y}\rangle\rangle$ donné par

$$
\mathrm{D}_{\psi}^{\mathrm{Y}}(v):=s_{\psi}^{\mathrm{Y}}(v)-v \mathbf{q} \pi_{\mathrm{Y}}(\psi)
$$

4.1.2. Pour calculer explicitement $s_{\psi}^{Y}(v)$, on doit choisir $w \in \mathbf{Q}\langle\langle\mathrm{X}\rangle\rangle$ tel que $\pi_{\mathrm{Y}}(w)=v$. Comme l'inclusion de $\mathbf{Q}\langle\langle\mathrm{Y}\rangle\rangle$ dans $\mathbf{Q}\langle\langle\mathrm{X}\rangle\rangle$ est une section de $\pi_{\mathrm{Y}}$, on peut prendre $w=v$.

Par exemple, on a $\pi_{\mathrm{Y}}\left(x_{1}\right)=y_{1,1}, \mathbf{q}\left(y_{1,1}\right)=y_{1,1}$ et $s_{\psi}\left(x_{1}\right)=\psi x_{1}+\left[x_{1}, \psi\right]=x_{1} \psi$, d'où l'on tire $\sigma_{\psi}^{\mathrm{Y}}\left(y_{1,1}\right)=\mathbf{q} \pi_{\mathrm{Y}}\left(x_{1} \psi\right)=y_{1,1} \mathbf{q} \pi_{\mathrm{Y}}(\psi)$. En d'autres termes :

Proposition 4.1.3. - Pour tout $\psi \in \mathbf{Q}\langle\langle\mathrm{X}\rangle\rangle$, on a $\mathrm{D}_{\psi}^{\mathrm{Y}}\left(y_{1,1}\right)=0$.

On constate également la propriété suivante :

Proposition 4.1.4. - Pour tout $\psi \in \mathbf{Q}\langle\langle\mathrm{X}\rangle\rangle$, l'opérateur $\mathrm{D}_{\psi}^{\mathrm{Y}}$ est une dérivation de $\mathbf{Q}\langle\langle\mathrm{Y}\rangle\rangle$.

Démonstration. — Par linéarité et une récurrence évidente, il suffit de démontrer :

$$
\mathrm{D}_{\psi}^{\mathrm{Y}}\left(y_{n, v} w\right)=\mathrm{D}_{\psi}^{\mathrm{Y}}\left(y_{n, v}\right) w+y_{n, v} \mathrm{D}_{\psi}^{\mathrm{Y}}(w),
$$

pour tout $(n, v) \in \mathbf{N}^{*} \times \Gamma$ et tout $w \in \mathbf{Q}\langle\mathrm{Y}\rangle$, homogène de couleur totale $\bar{w}$.

Remarquons d'abord que, pour $v \in \mathbf{Q}\langle\mathrm{Y}\rangle$, homogène de couleur totale $\bar{v}$, on a $\mathbf{p}(v w)=\mathbf{p}(v) t_{\bar{v}}(w)$, ce qui se vérifie facilement dans le cas où $v$ et $w$ sont des mots en Y (rappelons que $t_{v}$ désigne la substitution $\left.x_{0} \mapsto x_{0}, x_{\sigma} \mapsto x_{\sigma v}\right)$. Cela s'écrit aussi :

$$
\text { (4.1.4.1) } \quad v w=\mathbf{q}\left(\mathbf{p}(v) t_{\bar{\tau}} \mathbf{p}(w)\right) .
$$

D'après la définition de $\mathbf{p}$, on voit d'autre part que $\mathbf{q}(v)$ est homogène de couleur totale $\bar{v}$ si et seulement si tous les mots de $\mathrm{X}$ intervenant dans $v$ se terminent par $x_{v}$. 
En appliquant la définition de $\mathrm{D}_{\psi}^{\mathrm{Y}}$, on a

$$
\begin{aligned}
& \mathrm{D}_{\psi}^{\mathrm{Y}}\left(y_{n, \nu} w\right)=\mathbf{q} \pi_{\mathrm{Y}}\left[d_{\psi}\left(y_{n, \nu} t_{\nu} \mathbf{p}(w)\right)+\psi y_{n, \nu} t_{\nu} \mathbf{p}(w)\right]-y_{n, \nu} w \mathbf{q} \pi_{\mathrm{Y}}(\psi) \\
& =\mathbf{q} \pi_{\mathrm{Y}}\left[d_{\psi}\left(y_{n, \nu} t_{\nu} \mathbf{p}(w)\right)+\psi y_{n, \nu} t_{\nu} \mathbf{p}(w)-y_{n, \nu} t_{\nu} \mathbf{p}(w) t_{\nu \bar{w}} \psi\right] \\
& =\mathbf{q} \pi_{\mathrm{Y}}\left[d_{\psi}\left(y_{n, \nu}\right) t_{\nu} \mathbf{p}(w)+y_{n, \nu} t_{\nu} d_{\psi}(\mathbf{p}(w))+\psi y_{n, v} t_{\nu} \mathbf{p}(w)-y_{n, v} t_{\nu} \mathbf{p}(w) t_{\nu \bar{w}} \psi\right],
\end{aligned}
$$

cette dernière ligne s'obtenant en écrivant que $d_{\psi}$ est une dérivation et qu'elle commute, par définition, à $t_{v}$. On obtient de même

$$
\mathrm{D}_{\psi}^{\mathrm{Y}}\left(y_{n, v}\right)=\mathbf{q} \pi_{\mathrm{Y}}\left[d_{\psi}\left(y_{n, v}\right)+\psi y_{n, v}-y_{n, v} t_{v}(\psi)\right] .
$$

En revenant à la définition de $d_{\psi}$, on en déduit immédiatement que $\mathrm{D}_{\psi}^{\mathrm{Y}}\left(y_{n, v}\right)$ est égal à $\mathbf{q} \pi_{\mathrm{Y}}\left[-x_{0}^{n-1} t_{v}(\psi) x_{v}+\psi y_{n, v}\right]$ et est donc homogène de couleur totale $v$. On peut donc multiplier (4.1.4.3) à droite par $\mathbf{p}(w)$ en utilisant (4.1.4.1). Il vient :

$$
\mathrm{D}_{\psi}^{\mathrm{Y}}\left(y_{n, v}\right) w=\mathbf{q} \pi_{\mathrm{Y}}\left[d_{\psi}\left(y_{n, v}\right) t_{\nu} \mathbf{p}(w)+\psi y_{n, v} t_{\nu} \mathbf{p}(w)-y_{n, v} t_{v}(\psi) \tau_{v}(\mathbf{p}(w))\right] .
$$

D’un autre côté, on a, plus facilement

$$
\begin{aligned}
y_{n, \nu} \mathrm{D}_{\psi}^{\mathrm{Y}}(w) & =y_{n, \nu} \mathbf{q} \pi_{\mathrm{Y}}\left[d_{\psi}(\mathbf{p}(w))+\psi \mathbf{p}(w)-\mathbf{p}(w) t_{\bar{w}}(\psi)\right] \\
& =\mathbf{q} \pi_{\mathrm{Y}}\left[y_{n, \nu} t_{\nu} d_{\psi}(\mathbf{p}(w))+y_{n, \nu} t_{\nu}(\psi \mathbf{p}(w))-y_{n, \nu} t_{\nu}(\mathbf{p}(w)) t_{\nu \bar{w}}(\psi)\right] .
\end{aligned}
$$

La somme des seconds membres de (4.1.4.4) et (4.1.4.5) est bien celui de (4.1.4.2).

4.1.5. Remarques. - Dans le cas $\Gamma=\mathbf{1}$ des zêta multiples, l'opérateur $\mathrm{D}_{\psi}^{\mathrm{Y}}$ est simplement la restriction à $\mathbf{Q}\langle\langle\mathrm{Y}\rangle\rangle$ de la dérivation continue $\mathrm{D}_{\psi}$ définie $\operatorname{par} \mathrm{D}_{\psi}\left(x_{0}\right)=$ $\left[\psi, x_{0}\right]$ et $\mathrm{D}_{\psi}\left(x_{1}\right)=0$. Les propositions 4.1 .3 et 4.1.4 sont alors évidentes. Dans le cas général, on peut toujours considérer $\mathrm{D}_{\psi}^{\mathrm{Y}}$ comme la restriction de $v \mapsto \mathbf{q} d_{\psi} \mathbf{p}(v)-\psi \mathbf{q}(v)$ à $\mathbf{Q}\langle\langle\mathrm{Y}\rangle\rangle$, mais ceci n'est plus une dérivation de $\mathbf{Q}\langle\langle\mathrm{X}\rangle\rangle$.

4.2. Remontée. - Si $\psi_{1}$ et $\psi_{2}$ sont deux éléments de $\mathbf{Q}\langle\langle\mathrm{X}\rangle\rangle$ ayant la même image par $\pi_{\mathrm{Y}}$, il n'est pas vrai en général que $s_{\psi_{1}}^{\mathrm{Y}}$ et $s_{\psi_{2}}^{\mathrm{Y}}$ soient égales. On décrit ici une section de $\pi_{\mathrm{Y}}$ adaptée aux éléments de $\mathfrak{L} \mathfrak{i} \mathfrak{b}(\mathrm{X})$.

4.2.1. Notons $\partial_{x_{0}}$ la dérivée partielle par rapport à $x_{0}$ dans $\mathbf{Q}\langle X\rangle$, c'est-à-dire la dérivation qui envoie $x_{0}$ sur 1 et les $\left(x_{\sigma}\right)_{\sigma \in \Gamma}$ sur 0 . Elle est homogène pour toutes les graduations de $\mathbf{Q}\langle\mathrm{X}\rangle$ pour lesquelles les éléments de $\mathrm{X}$ sont homogènes, en particulier le poids et la longueur. On vérifie facilement qu'elle commute à $\mathbf{p}$ et que $\mathbf{Q}\langle\langle\mathrm{Y}\rangle\rangle$ est stable par $\partial_{x_{0}}$. 
Soit sec l'application de $\mathbf{Q}\langle\langle\mathrm{Y}\rangle\rangle$ dans $\mathbf{Q}\langle\langle\mathrm{X}\rangle\rangle$ définie par

$$
\sec (\psi):=\sum_{i \geq 0} \frac{(-1)^{i}}{i !} \partial_{x_{0}}^{i}(\psi) x_{0}^{i} .
$$

Pour $\psi \in \mathbf{Q}\langle\langle\mathrm{Y}\rangle\rangle$, on a clairement $\pi_{\mathrm{Y}} \sec \psi=\psi$. Tout élément $\psi$ de $\mathbf{Q}\langle\langle\mathrm{X}\rangle\rangle$ s'écrit de manière unique sous la forme $\sum_{i \geq 0} \psi_{i} x_{0}^{i}$ si l'on impose aux $\psi_{i}$ d'appartenir à $\mathbf{Q}\langle\langle\mathrm{Y}\rangle\rangle$, et $\psi_{0}$ n'est autre que $\pi_{\mathrm{Y}}(\psi)$. La condition $\partial_{x_{0}}(\psi)=0$ est donc équivalente à

$$
\sum_{i \geq 0} \partial_{x_{0}}\left(\psi_{i}\right) x_{0}^{i}+\sum_{i \geq 0}(i+1) \psi_{i+1} x_{0}^{i} .
$$

Les $\partial_{x_{0}}\left(\psi_{i}\right)$ appartenant à $\mathbf{Q}\langle\langle\mathrm{Y}\rangle\rangle$, ceci est encore équivalent à la relation de récurrence $(i+1) \psi_{i+1}+\psi_{i}=0$, pour tout $i \in \mathbf{N}$. On en déduit que les conditions $\partial_{x_{0}}(\psi)=0$ et $\psi=\sec \left(\psi_{0}\right)$ sont équivalentes. Autrement dit :

Proposition 4.2.2. - L'application sec : $\mathbf{Q}\langle\langle\mathrm{Y}\rangle\rangle \rightarrow$ ker $\partial_{x_{0}} \subset \mathbf{Q}\langle\langle\mathrm{X}\rangle\rangle$ est l'inverse de la restriction de $\pi_{\mathrm{Y}}$ à $\operatorname{ker} \partial_{x_{0}}$.

De la définition de $\partial_{x_{0}}$ découle par une récurrence immédiate que $\partial_{x_{0}}$ annule tout élément de $\mathfrak{L i b}(\mathrm{X})$ homogène de poids au moins 2. Pour $\psi \in \widehat{\mathfrak{L i b}}_{k}(\mathrm{X})$, on a donc simplement $\partial_{x_{0}}(\psi)=\left(\psi \mid x_{0}\right)$. De plus, on a $\partial_{x_{0}}\left(y_{1,1}^{n}\right)=0$, et $\operatorname{donc} \sec \left(y_{1,1}^{n}\right)=$ $y_{1,1}^{n}$. De tout cela s'ensuit :

Proposition 4.2.3. - Pour $\psi \in \mathfrak{d m r}(k)$, on a $\psi=\mathbf{p} \sec \left(\psi_{\star}\right)-\psi_{\text {corr }}$.

4.2.4. Propriétés de $\partial_{x_{0}}$ sur $\mathbf{Q}\langle\mathrm{Y}\rangle$. — La restriction de $\partial_{x_{0}}$ à $\mathbf{Q}\langle\langle\mathrm{Y}\rangle\rangle$ est la dérivation qui envoie $y_{n, v}=x_{0}^{n-1} x_{v}$ sur $(n-1) y_{n-1, v}$. Elle est donc homogène de degré $(-1,1)$ pour le poids coloré et de degré -1 pour la longueur.

Proposition 4.2.5. - Pour tous $(n, v) \in \mathbf{N}^{*} \times \Gamma$, on a $\partial_{x_{0}} u_{n, v}=(n-1) u_{n, v}$.

Démonstration. - Dans l'algèbre $\mathbf{Q}[t]\langle\langle\mathrm{Y}\rangle\rangle$, considérons la dérivation $\mathbf{Q}$-linéaire $\partial_{t}$ par rapport à $t$. Notons $\partial=\partial_{x_{0}}-t^{2} \partial_{t}$. Comme $t^{2}$ est central, $\partial$ est encore une dérivation.

Soit $\left(\psi_{n}\right)_{n \geq 0}$ une suite d'éléments homogènes de poids $n$ de $\mathbf{Q}\langle\mathrm{Y}\rangle$ et $\Psi:=$ $\sum_{n} \psi_{n} t^{n}$. On vérifie facilement que les conditions

$$
\text { [Pour tout } \left.n>0, \partial_{x_{0}}\left(\psi_{n}\right)=(n-1) \psi_{n-1}\right] \quad \text { et } \quad \partial \Psi=0
$$

sont équivalentes. La première est vérifiée pour $\psi_{n}=\sum_{v \in \Gamma} y_{n, v}$, et dans ce cas, la série $\Psi$ n'est autre que $\mathscr{Y}_{1}(t)$, avec les notations de 2.3.2. 
DOUBLES MÉLANGES DES POLYLOGARITHMES MULTIPLES AUX RACINES DE L’UNITÉ 219

Soit $\mathscr{U}(t)$ la série $\sum_{n, v} t^{n} u_{n, v}$. Par définition des $u_{n, v}$, on a $\mathscr{U}(t)=\log \left(\mathscr{Y}_{1}(t)\right)$. Comme $\mathscr{Y}_{1}(t)$ vérifie (4.2.5.1), en développant le logarithme et en utilisant que $\partial$ est une dérivation, on en déduit $\partial \mathscr{U}(t)=0$.

Pour tout $n>0$, on a donc $\partial_{x_{0}}\left(\psi_{n}\right)=(n-1) \psi_{n-1}$, avec $\psi_{n}=\sum_{v \in \Gamma} u_{n, v}$. La proposition en découle, car $\partial_{x_{0}}$ est homogène pour la couleur totale.

Comme l'ensemble des éléments primitifs de $\mathbf{Q}\langle\mathrm{Y}\rangle$ est l'ensemble des polynômes de Lie en les $u_{n, v}$, cela implique :

Corollaire 4.2.6. - L'ensemble des primitifs de $\mathbf{Q}\langle\langle\mathrm{Y}\rangle\rangle$ est stable par $\partial_{x_{0}}$.

On aurait aussi pu vérifier directement par calcul sur les $y_{n, v}$ que $\partial_{x_{0}}$ est une codérivation, redonnant ainsi ce corollaire.

4.3. Action par codérivation. - On établit ici par un calcul le résultat cidessous, dont la proposition 4.A sera une conséquence quasi-directe.

Proposition 4.3.1. - Pour tout $\psi \in \mathfrak{d} \mathfrak{m} \mathfrak{r}_{0}$, l'application $s_{\mathbf{p} \sec \left(\psi_{\star}\right)}^{\mathrm{Y}}$ est une codérivation.

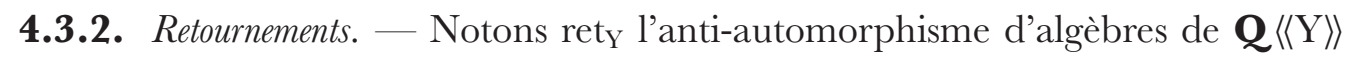
tel que $\operatorname{ret}_{\mathrm{Y}}\left(y_{n, v}\right)=y_{n, v^{-1}}$, pour $(n, v) \in \mathbf{N}^{*} \times \Gamma$. Par test sur les $y_{n, v}$, c'est un morphisme de cogèbres qui fixe 1, d'où en particulier :

Proposition 4.3.3. - L'ensemble des éléments primitifs de $\mathbf{Q}\langle\langle\mathrm{Y}\rangle\rangle$ est stable par ret . $_{\mathrm{Y}}$

Cet opérateur apparaît dans l'expression des $\mathrm{D}_{\psi}^{\mathrm{Y}}\left(y_{n, v}\right)$ lorsque $\mathrm{S}_{\mathrm{X}}(\psi)=-\psi$, où $\mathrm{S}_{\mathrm{X}}$ désigne l'antipode de $(\mathbf{Q}\langle\mathrm{X}\rangle, \cdot, \Delta)$, i.e. l'anti-automorphisme d'algèbres de $\mathbf{Q}\langle\mathrm{X}\rangle$ qui envoie $x_{\sigma}$ sur $-x_{\sigma}$. Cette condition est vérifiée en particulier si $\psi \in \mathfrak{L} \mathfrak{i b}(\mathrm{X})$.

Lemme 4.3.4. - Soit $\psi$ un élément homogène de poids p de $k\langle\mathrm{X}\rangle$ tel que $\mathrm{S}_{\mathrm{X}}(\psi)=-\psi$. Soient $\left(\psi_{i, \gamma}\right)_{0 \leq i \leq p, \gamma \in \Gamma}$ les éléments de $\mathbf{Q}\langle\langle\mathrm{Y}\rangle\rangle$ de couleur totale $\gamma$ caractérisés par :

$$
\psi=\sum_{i \geq 0} \mathbf{p}\left(\psi_{i, \gamma}\right) x_{0}^{i}
$$

Avec ces notations, on a pour tout $(n, v) \in \mathbf{N}^{*} \times \Gamma$ :

$$
\mathrm{D}_{\psi}^{\mathrm{Y}}\left(y_{n, v}\right)=\sum_{i \geq 0}\left(\psi_{i} y_{n+i, \nu \gamma^{-1}}+(-1)^{p} y_{n+i, \nu \gamma} \operatorname{ret}_{\mathrm{Y}}\left(\psi_{i \gamma}\right)\right)
$$


Démonstration. - On a successivement

$$
\begin{aligned}
s_{\psi}\left(y_{n, v}\right) & =s_{\psi}\left(x_{0}^{n-1} x_{\nu}\right)=\psi x_{0}^{n-1} x_{v}+x_{0}^{n-1} x_{v} t_{v}(\psi)-x_{0}^{n-1} t_{v}(\psi) x_{v}, \quad \text { d'où } \\
s_{\psi}^{\mathrm{Y}}\left(y_{n, v}\right) & =\mathbf{q}\left(\psi x_{0}^{n-1} x_{\nu}\right)+y_{n, v} \pi_{\mathrm{Y}} \psi-\mathbf{q}\left(x_{0}^{n-1} \psi x_{v}\right) .
\end{aligned}
$$

Le résultat voulu découle alors de la définition de $\mathrm{D}_{\psi}^{\mathrm{Y}}$ et du lemme immédiat cidessous, compte-tenu de $\mathrm{S}_{\mathrm{X}}(\psi)=-\psi$.

Lemme 4.3.5. - Pour tout élément $\psi=\sum_{i \geq 0, \gamma \in \Gamma} \mathbf{p}\left(\psi_{i, \gamma}\right) x_{0}^{i}$ de $\mathbf{Q}\langle\langle\mathrm{X}\rangle\rangle$, homogène de poids $p$, les $\psi_{i, \gamma}$ étant homogènes de couleur totale $\gamma$ dans $\mathbf{Q}\langle\langle\mathrm{Y}\rangle\rangle$ et tout $(n, v) \in \mathbf{N}^{*} \times \Gamma$, les formules suivantes sont valables:

$$
\begin{aligned}
\mathbf{q}\left(\psi x_{0}^{n-1} x_{\nu}\right) & =\sum_{i \geq 0, \gamma \in \Gamma} \psi_{i, \gamma} y_{n+i, \nu \gamma^{-1}} \\
\mathbf{q}\left(x_{0}^{n-1} t_{\nu} \mathrm{S}_{\mathbf{X}}(\psi) x_{\nu}\right) & =(-1)^{p} \sum_{i \geq 0} y_{n+i, \nu \gamma} \operatorname{ret}_{\mathrm{Y}}\left(\psi_{i, \gamma}\right) .
\end{aligned}
$$

4.3.6. Abréviations. — Étant donné que $s_{\mathbf{p} \sec \left(\psi_{\star}\right)}^{Y}$ dépend linéairement de $\psi$, pour démontrer la proposition 4.3.1, il suffit de traiter le cas où $\psi$ est un élément homogène de poids $p$ de $\mathfrak{d} \mathfrak{m r}_{0}$. On le supposera fixé dans la suite et on adoptera les notations suivantes :

- on abrège $\mathrm{D}_{\mathbf{p} \sec \left(\psi_{\star}\right)}^{\mathrm{Y}}$ en $\mathrm{D}$

- pour tout $(i, \gamma) \in \mathbf{N} \times \Gamma$, on note $\psi_{i, \gamma}$ la composante homogène de couleur totale $\gamma$ de $(-1)^{i} \partial_{x_{0}}^{i}\left(\psi_{\star}\right) / i$ ! et on pose $\chi_{i, \gamma}:=(-1)^{p} \operatorname{ret}_{\mathrm{Y}}\left(\psi_{i, \gamma}\right)$.

- on pose $z_{i, k}^{\gamma, v}:=\psi_{i, \gamma} y_{k, v \gamma^{-1}}+y_{k, v \gamma} \chi_{i, \gamma}$, pour tout $(i, k, \gamma, v) \in \mathbf{N}^{2} \times \Gamma^{2}$.

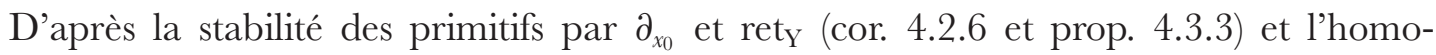
généité de $\Delta_{\star}$ pour la couleur totale, comme $\psi_{\star}$ est primitif, tous les $\psi_{i, \gamma}$ et $\chi_{i, \gamma}$ le sont.

4.3.7. Calcul de D sur les $y_{n, v}$. - D'après le corollaire $4.2 .3, \mathbf{p} \sec \left(\psi_{\star}\right)$ est la somme du polynôme de Lie $\psi$ de $\mathbf{Q}\langle\langle\mathrm{X}\rangle\rangle$ et de $\psi_{\text {corr }}$, par définition multiple scalaire de $\left(\psi \mid y_{p}\right) x_{1}^{p}$, et donc nul par la proposition 3.3 .3 si $p$ est pair. Il s'ensuit que $\mathbf{p} \sec \left(\psi_{\star}\right)$ est transformé en son opposé par $\mathrm{S}_{\mathrm{X}}$, ce qui permet d'appliquer le lemme 4.3.4. Avec les abréviations de 4.3.6, cela s'écrit, pour $n>0$ et $v \in \Gamma$ :

$$
\mathrm{D}\left(y_{n, v}\right)=\sum_{(i, \gamma) \in \mathbf{N} \times \Gamma} z_{i, i+n}^{\gamma, v}
$$

Ceci reste en fait vrai pour $n=0$, avec la convention (2.3.1.1) : c'est le cas particulier $k=0 \mathrm{du}$ lemme ci-dessous, qui interviendra également dans le calcul final. 
DOUBLES MÉLANGES DES POLYLOGARITHMES MULTIPLES AUX RACINES DE L'UNITÉ 221

Lemme 4.3.8. - Pour tout $(k, \kappa) \in \mathbf{N} \times \Gamma$, on a

(4.3.8.1) $\quad \sum_{i \geq k, \gamma \in \Gamma} z_{i, i-k}^{\gamma, \kappa}=0$.

Démonstration. - On a $\mathrm{D}\left(y_{1,1}\right)=0$ (prop. 4.1.3). Avec la formule (4.3.7.1) cela s'écrit

$$
\sum_{i \geq 0, \gamma \in \Gamma} z_{i, i+1}^{\gamma, 1}=0
$$

D’après la proposition 2.3.8, en appliquant la dérivation $\partial_{u_{k+1, \kappa^{-1}}}$ à cette égalité, on obtient :

$$
\sum_{i \geq k, \gamma \in \Gamma}\left(y_{i+1, \gamma^{-1}}\left(\psi_{i, \gamma} \mid y_{k+1, \kappa^{-1}}\right)+y_{i+1, \gamma}\left(\chi_{i, \gamma} \mid y_{k+1, \kappa^{-1}}\right)+z_{i, i-k}^{\gamma, \kappa}\right)=0 .
$$

Pour achever la démonstration du lemme, Il suffit donc de prouver l'égalité

$$
\sum_{i \geq k, \gamma \in \Gamma}\left(y_{i+1, \gamma^{-1}}\left(\psi_{i, \gamma} \mid y_{k+1, \kappa^{-1}}\right)+y_{i+1, \gamma}\left(\chi_{i, \gamma} \mid y_{k+1, \kappa^{-1}}\right)\right)=0 .
$$

Les $\psi_{i, \gamma}$ et $\chi_{i, \gamma}$ étant homogènes de poids $p-i$ et de couleurs totales respectives $\gamma$ et $\gamma^{-1}$, les termes de cette somme autres que $(i, \gamma)=(p-k-1, \kappa)$ et $(i, \gamma)=(p-k-1$, $\left.\kappa^{-1}\right)$ sont nuls; celle-ci vaut donc :

$$
\begin{aligned}
& y_{p-k, \kappa^{-1}}\left(\psi_{p-k-1, \kappa^{-1}} \mid y_{k, \kappa^{-1}}\right)+y_{p-k, \kappa^{-1}}\left(\chi_{p-k-1, \kappa^{-1}} \mid y_{k, \kappa^{-1}}\right) \\
& =\frac{(p-1) !}{k !} y_{p-k, \kappa^{-1}}\left(\psi_{\star} \mid y_{p, \kappa^{-1}}+(-1)^{p} y_{p, \kappa}\right),
\end{aligned}
$$

et ceci est nul par définition de $\mathfrak{d} \mathfrak{m} \mathfrak{r}_{0}$.

4.3.9. Démonstration de la proposition 4.3.1. - La multiplication à droite par $\psi_{\star}$ est une codérivation car $\psi_{\star}$ est primitif. Il suffit donc de démontrer que $\mathrm{D}=\mathrm{D}_{\mathbf{p} \sec \left(\psi_{\star}\right)}^{\mathrm{Y}}$ est une codérivation. Comme D est une dérivation, il suffit d'obtenir l'identité de codérivation sur les générateurs de $\mathbf{Q}\langle\langle Y\rangle\rangle$, i.e.

$$
\forall(n, v) \in \mathbf{N}^{*} \times \Gamma, \Delta_{\star} \mathrm{D}\left(y_{n, v}\right)=(\mathrm{Id} \otimes \mathrm{D}+\mathrm{D} \otimes \mathrm{Id}) \Delta_{\star}\left(y_{n, v}\right) .
$$

Abrégeons $a \otimes b+b \otimes a$ en $\operatorname{sym}(a \otimes b)$. La primitivité des $\psi_{i, \gamma}$ et $\chi_{i, \gamma}$ permet d'obtenir

$$
\Delta_{\star}\left(z_{i, j}^{\gamma, \nu}\right)=\sum_{\substack{k+l=i \\ \kappa \lambda=\nu}} \operatorname{sym}\left(y_{k, \kappa} \otimes z_{i, l}^{\gamma, \lambda}\right)
$$


Le premier membre de (4.3.9.1) est donc, en utilisant la formule (4.3.7.1) :

$$
\Delta_{\star} \mathrm{D}\left(y_{n, v}\right)=\sum_{\substack{i \geq 0 \\ \gamma \in \Gamma}} \sum_{\substack{k=0 \\ \kappa \in \Gamma}}^{i+n} \operatorname{sym}\left(z_{i, k}^{\gamma, \kappa} \otimes y_{n+i-k, \nu \kappa^{-1}}\right) .
$$

Évaluons le second membre de (4.3.9.1) :

$$
\begin{aligned}
& (\mathrm{D} \otimes \mathrm{Id}+\mathrm{Id} \otimes \mathrm{D}) \Delta_{\star}\left(y_{n, \nu}\right)=(\mathrm{D} \otimes \mathrm{Id}+\mathrm{Id} \otimes \mathrm{D}) \sum_{\substack{k+l=n \\
\kappa \lambda=v \\
\kappa=\nu}} y_{k, \kappa} \otimes y_{l, \lambda} \\
& =\sum_{\substack{k+l=n \\
\kappa \lambda=\nu}} \operatorname{sym}\left(\mathrm{D}\left(y_{k, \kappa}\right) \otimes y_{l, \lambda}\right)=\sum_{\substack{k=0 \\
\kappa \in \Gamma}}^{n} \sum_{\substack{i \geq 0 \\
\gamma \in \Gamma}} \operatorname{sym}\left(z_{i, i+k}^{\gamma, \kappa} \otimes y_{n-k, \nu \kappa^{-1}}\right) \\
& =\sum_{\substack{i \geq 0 \\
\gamma \in \Gamma}} \sum_{\substack{k=i \\
\kappa \in \Gamma}}^{i+n} \operatorname{sym}\left(z_{i, k}^{\gamma, \kappa} \otimes y_{n+i-k, \nu \kappa^{-1}}\right) .
\end{aligned}
$$

La différence des deux membres de (4.3.9.1) est donc

$$
\begin{aligned}
& \sum_{\substack{i \geq 0 \\
\gamma \in \Gamma}} \sum_{\substack{k=0 \\
\kappa \in \Gamma}}^{i-1} \operatorname{sym}\left(z_{i, k}^{\gamma, \kappa} \otimes y_{n+i-k, v \kappa^{-1}}\right)=\sum_{\substack{i \geq 0 \\
\gamma \in \Gamma}} \sum_{\substack{k=1 \\
\kappa \in \Gamma}}^{i} \operatorname{sym}\left(z_{i, i-k}^{\gamma, \kappa} \otimes y_{n+k, \nu \kappa^{-1}}\right) \\
& =\sum_{\substack{k \geq 1 \\
\kappa \in \Gamma}} \operatorname{sym}\left[\left(\sum_{\substack{i \geq k \\
\gamma \in \Gamma}} z_{i, i-k}^{\gamma, \kappa}\right) \otimes y_{n+k, \nu \kappa^{-1}}\right] .
\end{aligned}
$$

Or cette dernière expression est nulle d'après le lemme 4.3.8.

4.4. Preuve de la proposition 4.A. - Il nous reste essentiellement à vérifier que les termes correctifs se comportent bien.

4.4.1. Soient $\psi, v \in \mathbf{Q}\langle\langle\mathrm{X}\rangle\rangle$. On a $d_{\psi}\left(x_{1} v\right)=\left[x_{1}, \psi\right] v+x_{1} d_{\psi}(v)$, d'où l'on tire

$$
\text { (4.4.1.1) } \quad s_{\psi}\left(x_{1} v\right)=x_{1} s_{\psi}(v) \text {. }
$$

Soit $n$ un entier. La dérivation $d_{x_{1}^{n}}$ annule par définition $x_{0}$ et, pour tout $\sigma \in \Gamma$, on a $d_{x_{1}^{n}}\left(x_{\sigma}\right)=\left[x_{\sigma}, x_{\sigma}^{n}\right]=0$. On en déduit donc

$$
\text { (4.4.1.2) } \quad s_{x_{1}^{n}}(v)=x_{1}^{n} v \text {. }
$$

Il résulte de ces deux informations que $s_{\psi}$ et $s_{x_{1}^{n}}$ commutent. Tout ceci n'est que le cas particulier $k=\mathbf{Q}[\varepsilon]$ des remarques de 3.1.4.

On a $\mathbf{q} \pi_{\mathrm{Y}}\left(x_{1} v\right)=y_{1,1} \mathbf{q} \pi_{\mathrm{Y}}(v)$, d'où des formulations analogues pour les $s_{\psi}^{\mathrm{Y}}$. 
DOUBLES MÉLANGES DES POLYLOGARITHMES MULTIPLES AUX RACINES DE L'UNITÉ 223

4.4.2. Termes de longueur 1. - L'application bilinéaire $(\psi, \Phi) \mapsto s_{\psi}(\Phi)$ est homogène pour la longueur. Il en résulte si $\psi$ est sans terme constant que la partie de longueur 1 de $s_{\psi}^{n}(\Phi)$ est nulle pour $n>1$. De plus, si le terme constant de $\Phi$ vaut 1 , le terme en $y_{n, v}$ de $s_{\psi}(\Phi)$ est celui de $s_{\psi}(1)=\psi$. Ceci s'écrit également

$$
\left(\exp \left(s_{\psi}\right)(\Phi) \mid y_{n, v}\right)=\left(\psi+\Phi \mid y_{n, v}\right)
$$

De même, le terme de poids 1 de $\exp \left(s_{\psi}\right) \Phi$ est celui de $\psi+\Phi$.

Cela s'applique notamment au cas où $\psi$ et $\Phi$ sont respectivement une série et une exponentielle de Lie.

4.4.3. Crochet d'Ihara. - Rappelons que l'on a $s_{\psi}(1)=\psi$, et donc $\mathbf{q} \pi_{\mathrm{Y}}(\psi)=$ $s_{\psi}^{\mathrm{Y}}(1)$, pour tout $\psi \in \mathbf{Q}\langle\langle\mathrm{X}\rangle\rangle$.

Soient $\psi_{1}$ et $\psi_{2}$ deux éléments de $\mathfrak{d} \mathfrak{m} \mathfrak{r}_{0}$. L'algèbre de Lie libre $\mathfrak{L} \mathfrak{i} \mathfrak{b}_{k}(\mathrm{X})$ est stable pour le crochet d'Ihara (prop. 3.1.6). Celui-ci étant homogène pour le poids et la longueur, $<\psi_{1}, \psi_{2}>$ n'a aucun terme de poids 1 , et donc pas de terme en $x_{0}$ ni $x_{1}$, ni de longueur 1 et vérifie donc (3.3.3.1) pour tout $n$.

D'autre part, $s_{<\psi_{1}, \psi_{2}>}$ est le crochet de $s_{\psi_{1}}$ et $s_{\psi_{2}}$. Comme $\psi_{i}=\mathbf{p} \sec \psi_{i, \star}-\psi_{i \text {, corr }}$, pour $i=1,2$, on déduit de 4.4.1 l'égalité

$$
\begin{aligned}
& {\left[s_{\psi_{1}}, s_{\psi_{2}}\right]=\left[s_{\left.\mathbf{p} \sec \psi_{1, \star}, s_{\mathbf{p} \sec \psi_{2, \star}}\right], \quad \text { d'où }}\right.} \\
& {\left[s_{\psi_{1}}^{\mathrm{Y}}, s_{\psi_{2}}^{\mathrm{Y}}\right](1)=\left[s_{\mathbf{p} \sec \psi_{1, \star}}^{\mathrm{Y}}, s_{\mathbf{p} \sec \psi_{2, \star}}^{\mathrm{Y}}\right](1) .}
\end{aligned}
$$

Le membre de gauche de cette dernière égalité vaut $\mathbf{q} \pi_{\mathrm{Y}}\left(<\psi_{1}, \psi_{2}>\right)$. Le membre de droite est l'image de 1 par une codérivation pour $\Delta_{\star}$ : c'est donc un élément primitif pour $\Delta_{\star}$. On a donc prouvé que $\mathfrak{d} \mathfrak{m} \mathfrak{r}_{0}$ est une sous-algèbre de Lie de $\mathfrak{m t}$.

4.4.4. Fin de la preuve pour $\underline{\mathrm{DMR}}$. - Soit $\Phi \in \underline{\mathrm{DMR}}(k)$ et $\psi \in \mathfrak{d m r}_{0}(k)$. Il s'agit de prouver que $\exp ^{\circledast}(\psi) \circledast \Phi=\exp \left(s_{\psi}\right)(\Phi)$ appartient à $\underline{\operatorname{DMR}}(k)$.

Le coefficient de $x_{0}\left(\right.$ resp. $\left.x_{1}\right)$ dans $\exp \left(s_{\psi}\right)(\Phi)$ est nul, car c'est la somme de celui de $\psi$ et celui de $\Phi$. On sait déjà (prop. 3.1.6) que $\exp \left(s_{\psi}\right)(\Phi)$ est diagonal pour $\Delta$.

L'exponentielle d'une codérivation est un morphisme de cogèbres. L'élément

$$
\mathrm{G}:=\exp \left(s_{\mathbf{p} \sec \left(\psi_{\star}\right)}^{\mathrm{Y}}\right)\left(\Phi_{\star}\right)
$$

est donc diagonal pour $\Delta_{\star}$. Comme $\mathbf{p} \sec \left(\psi_{\star}\right)$ et $\psi\left(\right.$ resp. $\Phi_{\star}$ et $\left.\mathbf{q} \pi_{\mathrm{Y}}(\Phi)\right)$ sont égales à l'addition (resp. la multiplication à gauche) d'une série ne dépendant que de $x_{1}\left(=y_{1,1}\right)$ près, on déduit des remarques de 4.4.1 que $\mathrm{G}$ et $\mathbf{q} \pi_{\mathrm{Y}} \exp \left(s_{\psi}\right)(\Phi)$ sont égales, à multiplication à gauche près par une série ne dépendant que de $y_{1,1}$. Celle-ci est alors automatiquement $\left[\exp \left(s_{\psi}\right)(\Phi)\right]_{\text {corr }}$, d'après la proposition 2.4.14. 
4.4.5. Distribution. - Soient $\Phi_{1}, \Phi_{2}$ deux séries de termes constants 1 et satisfaisant, pour tout diviseur $d$ de l'ordre de $\Gamma$ à (3.2.1.3) : on a $\mathrm{S}_{k} \in k \llbracket x_{1} \rrbracket$ tel que $p_{*}^{d}\left(\Phi_{k}\right)=\mathrm{S}_{k} \cdot i_{d}^{*}\left(\Phi_{k}\right)$ pour $k \in\{1,2\}$.

Les applications $i_{d}^{*}$ et $p_{*}^{d}$ sont des morphismes de schémas en groupes de $\operatorname{MT}(\Gamma)$ dans $\operatorname{MT}\left(\Gamma^{d}\right)$. Comme les $\mathrm{S}_{k}$ sont de plus centrales dans $\mathrm{MT}\left(\Gamma^{d}\right)$, la relation de distribution vaut pour $\Phi_{1} \circledast \Phi_{2}$, à multiplication à gauche près par $\mathrm{S}_{1} \mathrm{~S}_{2}$, qu'on calcule facilement. Si les $\Phi_{k}$ sont des exponentielles de Lie, sans termes en $x_{0}$ ni $x_{1}$, on conclut plus rapidement par les arguments de 2.5.4.

Si $\psi$ est une série de Lie vérifiant (3.3.1.3), $p_{*}^{d}(\psi)=\mathrm{S}+i_{d}^{*}(\psi)$, où $\mathrm{S}$ ne dépend que de $x_{1}$, il est clair pour les mêmes raisons que $\exp ^{\circledast} \psi$ vérifie (3.2.1.3), si les coefficients de $x_{0}$ et $x_{1}$ dans $\psi$ sont nuls.

4.4.6. Poids un et fibres. - Soit $\iota$ un morphisme de groupes $\Gamma \rightarrow \mathbf{G}^{*}$. Les équations (3.2.1.4) et (3.2.1.5) concernent des coefficients du type $(\Phi \mid \alpha)$, avec $\alpha$ homogène de longueur 1 . Pour tous ces $\alpha$, on a par définition $(\psi \mid \alpha)=0$ si $\psi$ appartient à $\mathfrak{d} \mathfrak{m r}_{0}(k)$, et donc $\left(\exp \left(s_{\psi}\right)(\Phi) \mid \alpha\right)=(\Phi \mid \alpha)$, d'après 4.4.2. Ceci donne la stabilité des relations de poids un et le point iii) de la proposition 4.A.

\section{Transitivité et conséquences}

\subsection{Approximations successives}

5.1.1. Il est classique d'interpréter les actions de $\mathbf{G}_{\mathrm{m}}$ en termes $\mathbf{Z}$-graduations. De même, une action du monoïde multiplicatif $\mathbf{A}^{1}$ sur un schéma affine $\mathrm{P}=\operatorname{Spec}(\mathrm{A})$ équivaut à la donnée d'une $\mathbf{N}$-graduation sur $\mathrm{A}$ : le morphisme $\mathrm{A} \rightarrow \mathrm{A} \otimes \mathbf{Q}[t]$ définissant l'action est $a \longmapsto \sum_{n} a_{n} \otimes t^{n}$, où $a_{n}$ désigne la composante homogène de degré $n$ de A.

On supposera dans tout ce qui suit que les composantes homogènes de A sont de dimension finie. La multiplication de A fait alors du dual gradué de $\mathrm{A}$ une cogèbre graduée $(\mathrm{C}, \varepsilon, \Delta)$ dont on notera $\mathrm{C}_{n}$ la composante homogène de degré $n$. Étendons $\Delta$ et $\varepsilon$ au complété $\widehat{\mathrm{C}}(k)$, qui n'est autre que le dual ordinaire de $\mathrm{A} \otimes k$. L'ensemble des $k$-points de $\mathrm{P}$ s'interprète alors comme l'ensemble des éléments diagonaux de $(\widehat{\mathrm{C}}(k), \varepsilon, \Delta)$. Dans ce cadre, $\lambda \in k=\mathbf{A}^{1}(k)$ agit sur la composante homogène de degré $n$ de $\widehat{\mathrm{G}}(k)$ par multiplication par $\lambda^{n}$, et ceci redonne l'action de $\mathbf{A}^{1}$ sur $\mathrm{P}$.

5.1.2. Soient respectivement $\mathrm{C}^{(n)}$ et $(\mathrm{C} \otimes \mathrm{C})^{(n)}$ les $n^{\text {èmes }}$ quotients des filtrations de $\widehat{\mathrm{C}}(k)$ et $(\widehat{\mathrm{C}} \widehat{\otimes} \widehat{\mathrm{C}})(k)$. On notera dans les deux cas $\pi^{(n)}$ pour la projection correspondante. Il sera pratique d'identifier le $k$-module $\mathrm{C}^{(n)}(k)$ à $\oplus_{i=0}^{n} \mathrm{C}_{i} \otimes k$ et, de même, $(\mathrm{C} \otimes \mathrm{G})^{(n)}(k)$ à $\oplus_{i+j \leq n}\left(\mathrm{C}_{i} \otimes \mathrm{C}_{j} \otimes k\right)$. 
DOUBLES MÉLANGES DES POLYLOGARITHMES MULTIPLES AUX RACINES DE L’UNITÉ 225

Soit $\mathrm{P}^{(n)}(k)$ l'ensemble des éléments $\Phi$ de $\mathrm{C}^{(n)}(k)$ qui sont diagonaux modulo des termes de degré $n+1$, i.e. qui vérifient

$$
\text { (5.1.2.1) } \Delta \Phi=\pi^{(n)}(\Phi \otimes \Phi) \quad \text { et } \quad \varepsilon(\Phi)=1 \text {. }
$$

On retrouve $\mathrm{P}$ comme limite projective des $\mathrm{P}^{(n)}$. Par ailleurs, $\mathrm{P}^{(n)}$ est le spectre de la sous-algèbre de A engendrée par ses composantes homogènes de degré au plus $n$.

5.1.3. Par définition, un $k$-point de l'espace tangent $\mathrm{T}_{\Phi} \mathrm{P}$ à $\mathrm{P}$ au voisinage de $\Phi \in \mathrm{P}(\mathbf{Q})$ est un élément $\psi$ de $\mathrm{Y}(k)$ tel que $\Phi+\varepsilon \psi$ soit un $k[\varepsilon]$-point de $\mathrm{P}$, i.e. diagonal dans $\mathrm{Y}(k[\varepsilon])$. Cela se traduit par la condition

$$
\text { (5.1.3.1) } \quad \Delta \psi=\Phi \otimes \psi+\psi \otimes \Phi \text {. }
$$

Si $\Phi$ est fixe sous l'action de $\mathbf{A}^{1}$, cet espace tangent est stable par $\mathbf{A}^{1}$, et est donc un schéma vectoriel associé à un espace vectoriel gradué. Plus concrètement, les composantes homogènes de degré $>0$ de $\Phi$, vu comme élément de $\widehat{\mathrm{C}}(k)$, sont nulles. Par homogénéité de $\Delta$, les composantes homogènes d'un élément $\psi$ de $\mathrm{T}_{\Phi}(\mathrm{P})$ sont encore dans $\mathrm{T}_{\Phi}(\mathrm{P})$, et $\psi$ en est la somme infinie. Si l'on prefère voir $\Phi$ comme un morphisme d'algèbres $\mathrm{A} \rightarrow k$, cela fait de $\mathrm{T}_{\Phi} \mathrm{P}(k)$ l'ensemble des $\Phi$-dérivations de $\mathrm{A}$ dans $k$.

5.1.4. Action d'une algèbre de Lie. - Soit $\mathfrak{g}$ une algèbre de Lie graduée. Une action homogène de $\exp (\mathfrak{g})$ sur $\mathrm{P}$ donne lieu, pour tout $k$-point de $\exp (\mathfrak{g})$ à un endomorphisme de la cogèbre $\widehat{\mathrm{C}}(k)$. En exprimant ceci pour les nombres duaux $k[\varepsilon]$, on trouve une action, homogène, de $\mathfrak{g}$ sur $\widehat{\mathrm{C}}$ par codérivations qu'on notera encore $(\psi, v) \mapsto s_{\psi}(v)$. Si $v \in \mathrm{P}(k)$, on voit que $s_{\psi}(v)$ appartient à $\mathrm{T}_{v}(\mathrm{P})(k)$, d'après $(5.1 .3 .1)$. Enfin, l'action de $\exp (\psi)$ sur $\widehat{\mathrm{C}}(k)$ se fait $\operatorname{par} \exp \left(s_{\psi}\right)$.

Proposition 5.1.5. - Soient $\mathrm{P}$ un schéma affine, du type considéré en 5.1.1, $\mathrm{P} \stackrel{\alpha}{\longrightarrow} \mathbf{A}^{1}$ un morphisme, $\mathfrak{g}$ une $\mathbf{Q}$-algèbre de Lie graduée, à degrés strictement positifs, et une action de $\exp (\mathfrak{g})$ sur $\mathrm{P}$. On note $\mathrm{P}_{\lambda}$ la fibre de $\alpha$ au-dessus de $\lambda \in k$ et on fait les hypothèses :

i) Homogénéité de $\alpha$ : le diagramme ci-dessous est commutatif.

$(5.1 .5 .1)$

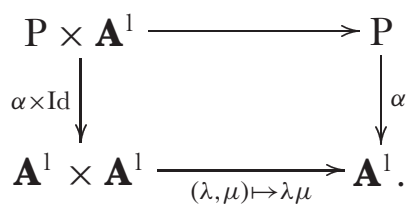

ii) Il existe un point fixe, noté 1 , de $\mathrm{P}$ sous l'action de $\mathbf{A}^{1}$ et un seul. Il est rationnel. Soit $\mathfrak{x}$ l'espace tangent à $\mathrm{P}$ au voisinage de 1 .

iii) L'action de $\mathrm{G}$ sur $\mathrm{P}$ est homogène et commute à $\alpha$. 
iv) L'application $\psi \mapsto s_{\psi}(1)$ est bijective de $\mathfrak{g}$ sur $\mathfrak{x}_{0}$.

v) Il existe un $\mathbf{Q}$-anneau $\mathbf{K}$ et $\lambda \in \mathbf{K}$ inversible tel que $\mathrm{P}_{\lambda}(\mathbf{K})$ soit non-vide.

Sous ces hypothèses, l'action de $\exp (\mathfrak{g})$ sur chaque $\mathrm{P}_{\lambda}$ est libre et transitive. De plus, $\mathrm{P}_{\lambda}(k)$ est non-vide pour tout $k$ et tout $\lambda \in k$.

Le point fixe 1 est nécessairement dans $\mathrm{P}_{0}$ et on a en particulier $\mathrm{P}_{0}=\exp (\mathfrak{g})(1)$. La condition ii) est équivalente au fait que la composante homogène de degré 0 de l'algèbre affine de $\mathrm{P}$ est réduite à $\mathbf{Q}$.

5.1.6. Preuve du théorème. - Le théorème I découle directement de la proposition 5.1.5, appliquée à $\mathrm{P}=\mathrm{DMR}^{\iota}$ ou $\mathrm{DMRD}^{\imath}$ :

On munit le schéma MT (séries formelles à terme constant 1) de l'action de $\mathbf{A}^{1}$ définie par le poids, et sur $\mathbf{A}^{1}$ l'action par multiplication. On laisse le lecteur vérifier que $\mathrm{P}$ est alors stable sous l'action diagonale de $\mathbf{A}^{1}$ sur $\mathbf{A}^{1} \times \mathrm{MT}$.

L'hypothèse de finitude est déjà vraie pour $\mathbf{A}^{1} \times \mathrm{MT}$. On pose $\mathfrak{g}=\mathfrak{d} \mathfrak{m} \mathfrak{r}_{0}$ (respectivement $\mathfrak{g}=\mathfrak{d} \mathfrak{m r} \mathfrak{d}_{0}$ ), munie du crochet d'Ihara et l'action donnée par la proposition 4.A. Les hypothèses i) et ii) et iii) sont évidentes. La flèche $\mathfrak{g} \rightarrow \mathfrak{x}_{0}: \psi \mapsto \sigma_{\psi}(1)$ est l'identité, d'où iv). Enfin v) est donnée par $\mathscr{I}^{\iota}$, élément de $\operatorname{DMRD}_{2 i \pi}^{\iota}(\mathbf{G})$.

Le reste de cette sous-section est consacré à la preuve de la proposition 5.1.5.

\subsection{Démonstration de la proposition 5.1 .5}

5.2.1. Le morphisme $\alpha$ donne un morphisme de cogèbres $\widehat{\mathrm{C}}(k) \rightarrow k \llbracket t \rrbracket$. Par l'hypothèse d'homogénéité, il commute aux $\pi^{(n)}$.

La formule (5.1.2.1) implique en particulier que le terme de degré 0 d'un élément de $\mathrm{P}^{(n)}(k)$ est diagonal. Il est donc égal à 1 , par l'hypothèse ii).

Proposition 5.2.2. - la différence de deux éléments de $\mathrm{P}_{\lambda}^{(n+1)}(k)$ ayant même image par $\pi^{(n)}$ est un élément homogène de degré $n+1$ de $\mathfrak{x}_{0}(k)$.

Si un élément de $\mathrm{P}^{(n)}(\mathbf{Q})$ admet un relevé dans $\mathrm{P}^{(n+1)}(k)$, il en admet un dans $\mathrm{P}^{(n+1)}(\mathbf{Q})$.

Démonstration. — Écrivons les composantes homogènes d'un élément $\Phi \in \mathrm{P}^{(n)}(k)$ :

$$
\Phi=1+\Phi_{1}+\cdots+\Phi_{n} .
$$

La condition portant sur un élément $\Phi_{n+1}$ de $\mathrm{Y}(k)$, homogène de degré $n+1$, pour que $\Phi+\Phi_{n+1}$ appartienne à $\mathrm{P}^{(n+1)}$ s'écrit :

$$
\Delta\left(\Phi_{n+1}\right)-1 \otimes \Phi_{n+1}-\Phi_{n+1} \otimes 1=\sum_{\substack{k+l=n+1 \\ k,>0}} \Phi_{k} \otimes \Phi_{l} .
$$

Sous cette forme, la première assertion est évidente, compte-tenu de la caractérisation (5.1.3.1) de $\mathfrak{x}(k)$ et de la linéarité de $\widehat{\mathrm{C}}(k) \rightarrow k \llbracket t \rrbracket$. La seconde revient à dire qu'un 
système linéaire à coefficients rationnels ayant une solution dans $k$ admet une solution dans $\mathbf{Q}$.

On définit pour tout $n$ une action de $\exp (\mathfrak{g}) \operatorname{sur} \pi^{(n)}(\mathrm{C}) \subset \widehat{\mathrm{C}}$ par l'action sur $\widehat{\mathrm{C}}$, suivie de $\pi^{(n)}$. Avec l'homogénéité et le fait que $s_{\psi}$ est une codérivation, on voit que $\mathrm{P}^{(n)}$ est stable par cette action. De plus, si $\psi$ est homogène de degré $n$, l'action de $\exp \left(s_{\psi}\right)$ sur $\mathrm{P}^{(n)}$ est simplement l'addition de $s_{\psi}(1)$.

Proposition 5.2.3. - Soit $k$ un $\mathbf{Q}$-anneau et $\lambda \in k$. Si $\mathrm{P}_{\lambda}(k) \neq \varnothing$, l'action de $\exp (\mathfrak{g})$ est libre et transitive sur chaque $\mathrm{P}_{\lambda}^{(n)}(k)$ et sur $\mathrm{P}_{\lambda}(k)$.

Démonstration. - On raisonne par récurrence, le cas $n=0$ étant trivial. Supposons le résultat établi pour un entier $n$. Tout d'abord, $\mathrm{P}^{(n+1)}(k)$ contient $\pi^{(n+1)}(\mathrm{P}(k))$ et n'est donc pas vide. Soient $\Phi_{1}^{(n+1)}$ et $\Phi_{2}^{(n+1)}$ deux éléments de $\mathrm{P}_{\lambda}^{(n+1)}(k)$. Notons $\Phi_{1}^{(n)}$ et $\Phi_{2}^{(n)}$ leurs images par $\pi^{(n)}$. Par hypothèse de récurrence, il existe un unique $\psi \in \mathfrak{g}(k)$ tel que $\Phi_{2}^{(n)}=\pi^{(n)} \exp \left(s_{\psi}\right) \Phi_{1}^{(n)}$.

Soit $\Psi$ l'image de $\Phi_{1}^{(n+1)}$ par $\pi^{(n+1)} \exp \left(s_{\psi}\right)$. C'est un élément de $\mathrm{P}_{\lambda}^{(n+1)}(k)$, dont l'image par $\pi^{(n)}$ est $\Phi_{2}^{(n)}$. D'après la proposition 5.2.2, $\Phi_{2}^{(n+1)}-\Psi$ est un élément de $\mathfrak{x}_{0}(k)$, homogène de degré $n+1$, donc de la forme $s_{\psi_{n+1}}(1)$, pour un unique $\psi_{n+1} \in$ $\mathfrak{g}(k)$. Comme $\pi^{(n+1)} \exp \left(s_{\psi_{n+1}}\right)$ est précisément l'addition de $s_{\psi_{n+1}}(1)$, ceci achève la récurrence. Le passage à la limite ne pose pas de problème. de $\mathrm{P}_{0}$.

Comme 1 est élément de $\mathrm{P}_{0}(\mathbf{Q})$, on a donc déjà l'isomorphisme de $\exp (\mathfrak{g})$ et

Proposition 5.2.4. - Il existe un élément de $\mathrm{P}_{1}(\mathbf{Q})$.

Démonstration. - Il suffit de montrer pour tout $n$ que tout $\Phi \in \mathrm{P}_{1}^{(n)}(\mathbf{Q})$ peut se relever à $\mathrm{P}_{1}^{(n+1)}(\mathbf{Q})$. De l'hypothèse v), on déduit par l'action de $\mathbf{A}^{1}$ un élément $\Psi$ de $\mathrm{P}_{1}(\mathbf{K})$. La proposition précédente fournit $\psi \in \mathfrak{g}(\mathbf{K})$ tel que $\pi^{(n)} \exp \left(s_{\psi}\right)$ envoie $\pi^{(n)}(\Psi)$ sur $\Phi$. L'image de $\pi^{(n+1)}(\Psi)$ par $\pi^{(n+1)} \exp \left(s_{\psi}\right)$ est un relevé à coefficients dans $\mathbf{K}$ de $\Phi$. On conclut avec la proposition 5.2.2.

Pour achever la preuve de la proposition 5.1.5, il reste à vérifier que $\mathrm{P}_{\lambda}(k)$ n'est jamais vide. Il suffit pour cela de considérer l'action homogène de $\lambda$ sur un élément de $\mathrm{P}_{1}(\mathbf{Q})$.

\subsection{Conséquences du théorème}

5.3.1. Théorème d'Écalle. - Fixons un plongement $\iota: \Gamma \rightarrow \mathbf{C}^{*}$. La donnée de $\Phi \in \operatorname{DMRD}_{1}(\mathbf{Q})$ équivaut à celle d'un isomorphisme $\mathbf{A}^{1}$-équivariant

$$
\mathbf{A}^{1} \times \mathfrak{d m r d} \mathfrak{d}_{0} \stackrel{\sim}{\longrightarrow} \mathrm{DMRD}^{\iota} .
$$

L'algèbre affine de $\mathrm{DMRD}^{\iota}$, qu'on peut voir comme engendrée par des symboles formels représentant les valeurs de polylogarithmes multiples sur $\iota(\Gamma)$ soumis aux rela- 
tions DMRD et de poids un auxquels on a adjoint une valeur formelle de $2 i \pi$, i.e. un symbole $t$ tel que $-t^{2} / 24=(\zeta(2)$ formel), est donc isomorphe, comme algèbre graduée, au produit tensoriel de $\mathbf{Q}[t]$ et de l'algèbre symétrique formée sur le dual gradué de $\mathfrak{d} \mathfrak{m} \mathfrak{r} \mathfrak{d}$. Un résultat analogue vaut pour $\mathrm{DMR}^{\iota}$, et plus généralement pour tout schéma ayant la propriété (M) de l'introduction.

Pour $\Gamma=\mathbf{1}$, ceci est une variante du théorème d'Écalle [14], dont la démonstration repose en partie sur des méthodes similaires (linéarisation par une algèbre de Lie isomorphe à $\mathfrak{d} \mathfrak{m} \mathfrak{r} \mathfrak{d}, c f$. [33, Appendice A]). La version du théorème I établie dans [33] est très légèrement plus forte : elle revient à ne pas adjoindre de $2 i \pi$ formel.

5.3.2. Indécomposables de Drinfel'd. - Les propriétés énumérées en 2.5.3 s'appliquent en particulier aux automorphismes de $\Gamma$, qui fournissent donc par image directe des automorphismes de $\underline{\mathrm{DMRD}}, \mathfrak{d} \mathfrak{m} \mathfrak{r} \mathfrak{d}$, etc. On note dans la suite $\mathrm{N}$ l'ordre de $\Gamma$.

Considérons la substitution «Frobenius à l'infini» $\mathrm{F}: x_{0} \mapsto-x_{0} ; x_{\sigma} \mapsto-x_{\sigma^{-1}}$. Notant inv l'inversion de $\Gamma$, on voit que $\mathrm{F}$ est la composée de inv* et de l'action homogène de -1 sur MT. De plus, elle fixe tous les $x_{\sigma}-x_{\sigma^{-1}}$, ainsi que $x_{0} x_{1}$. On en déduit immédiatement qu'elle laisse tous les $\operatorname{DMRD}_{\lambda}^{\iota}$ stables.

Par le théorème $\mathrm{I}$, il existe donc $\psi_{\iota} \in \mathfrak{d} \mathfrak{m} \mathfrak{r d}(\mathbf{G})$, tel que l'on ait $\mathrm{F}\left(\mathscr{I}^{\iota}\right)=$ $\exp \left(-s_{\psi_{\iota}}\right)\left(\mathscr{I}^{\iota}\right)$. De $\mathrm{F}^{2}=\mathrm{Id}$, on déduit facilement que $\mathrm{F}\left(\psi_{\iota}\right)=-\psi_{\iota}$. Les $\psi_{\iota}$ sont permutés par les automorphismes de $\Gamma$ et égaux au signe près à leurs images par l'inversion. On les indice par le choix d'un $\iota$ dans chaque orbite de l'inversion.

Soit $\psi_{n, l}$ la composante homogène de degré $n$ de $\psi_{\iota}$. Dans le cas $\mathrm{N}=1$, les $\psi_{n}$ sont les éléments irréductibles de $\mathfrak{g r t}_{1}(\mathbf{G})$ exhibés par Drinfel'd [13, p. 860]. D'après [11], les composantes homogènes non nulles des $\psi_{n, l}$ engendrent en général l'image dans $\mathfrak{m t}$ de l'algèbre de Lie du groupe motivique $\mathscr{U}_{\mathrm{gr}}$. Appliquant la propriété (M) de l'introduction dans les deux cas, on obtient que la variété des relations d'origine motivique est incluse dans DMRD. Autrement dit, les relations DMRD sont d'origine motivique.

On calcule facilement le terme de longueur 1 des $\psi_{n, \iota}$, en utilisant 4.4.2 :

Dans le cas réel $\mathrm{N} \leq 2$, l'inversion de $\Gamma$ est l'identité et $\mathrm{F}$ se réduit à l'action homogène de -1 . La série $\psi$ est impaire. Pour $n \geq 1$, son terme de longueur 1 et de poids $n$ vaut $2 \zeta(2 n+1) y_{2 n+1,1}$. On a $\psi_{1}=0$ pour $\mathrm{N}=1$ et $\psi_{1}=2 \log (2) y_{1,-1}$ pour $\mathrm{N}=2$.

Dans le cas général, le terme de longueur 1 de $\psi_{n, l}$ vaut

$$
\sum_{\sigma \in \Gamma}\left(\mathrm{L}_{n}(\iota(\sigma))-(-1)^{n} \mathrm{~L}_{n}\left(\iota\left(\sigma^{-1}\right)\right)\right) y_{n, \sigma} .
$$

Les $\psi_{n, \iota}$ non nuls ont donc un terme de longueur 1 non nul. Ils sont indécomposables, car le crochet d'Ihara est homogène pour la longueur. 
DOUBLES MÉLANGES DES POLYLOGARITHMES MULTIPLES AUX RACINES DE L’UNITÉ 229

Problème 1. - Pour quelles valeurs de $\mathrm{N}$ les $\psi_{n, \iota}$ engendrent-ils $\mathfrak{d} \mathfrak{m r} \mathfrak{d}_{0}$ ? Celle-ci est elle une algèbre de Lie libre?

Comme mentionné dans l'introduction, on n'attend pas en général de réponse positive. Pour $\mathrm{N}=1$, ce problème est une variante des questions de Drinfel'd [13] à propos de $\mathfrak{g r t}_{1}$, parfois qualifiée de «conjecture de Deligne-Drinfel'd.» La première question de la variante pour l'image de l'algèbre de Lie du complété pro- $\ell$ de $\operatorname{Gal}(\overline{\mathbf{Q}} / \mathbf{Q})$ dans $\mathfrak{g r t}_{1}\left(\mathbf{Q}_{\ell}\right)$ (conjecture de Deligne-Ihara) a été résolue par Hain et Matsumoto [22].

L’algèbre de Lie de $\mathscr{U}_{\mathrm{gr}}$ est libre, engendrée par des éléments dont les images dans $\mathfrak{m} t$ sont les $\psi_{\iota, n}$ [11]. Par le théorème $\mathrm{I}$, il en résulte que $\mathbf{M}^{\iota}$ est incluse dans $\mathrm{DMRD}^{\iota}$, i.e. que les relations d'origine motivique impliquent les relations DMRD. La première question ci-dessus est celle de la réciproque ; la seconde est équivalente à l'injectivité de $\mathscr{U}_{\text {gr }} \rightarrow$ MT.

Deligne a prouvé la liberté pour $\mathrm{N} \in\{2,3,4\}$ (non publié). Pour $\mathrm{N}=1$, on a obtenu par ordinateur une réponse positive aux deux questions jusqu'en poids 19 ; cela fera l'objet d'un autre article [15]. Écalle a annoncé avoir une réponse positive aux deux questions dans le cas $\mathrm{N}=2$ (preuve non publiée), à des différences de langage près.

Les conjectures de transcendance et la conjecture de Deligne-Drinfel'd amènent également à la question transversale :

Problème 2. - Les algèbres de Lie $\mathfrak{g r t}_{1}$ et $\mathfrak{d} \mathfrak{m} \mathfrak{r}_{0}$ sont elles égales?

Une réponse positive ramènerait la construction explicite d'associateurs rationnels à celle d'éléments de $\operatorname{DMR}_{1}(\mathbf{Q})$, ce qu'on espère être plus facile.

\section{Index des notations}

$$
\begin{array}{ll}
\zeta\left(s_{1}, \ldots, s_{r}\right), \mathrm{L}_{s_{1}, \ldots, s_{r}} & \text { Introduction } \\
k, k\langle\mathrm{Z}\rangle, k\left\langle\langle\mathrm{Z}\rangle,, \mathfrak{L i b}_{k}(\mathrm{Z}), \mathrm{U}_{k} \mathfrak{g},(\cdot \mid \cdot)\right. & 1.6 \\
\mathscr{H}(\mathrm{D}), \operatorname{DivLog}{ }_{\mathrm{D}}, \operatorname{DivLog} \mathbf{N}, \mathrm{As} & 2.1 .1 \\
\mathrm{cmp} & 2.1 .5 \\
\mathrm{I}_{\gamma}, \mathscr{I}_{\gamma}, x_{\sigma}, \mathrm{X}_{\Gamma}, \mathrm{X} & 2.2 .1,2.2 .3 \\
y_{n, v}, \mathrm{Y}, \pi_{\mathrm{Y}}, \mathbf{p}, \mathbf{q} & 2.2 .5,2.2 .7 \\
\mathbf{Q}\langle\mathrm{X}\rangle_{\mathrm{cv}}, \pi_{\mathrm{cv}}, \mathscr{I}_{\mathrm{cv}}, \mathbf{Q}\langle\mathrm{Y}\rangle_{\mathrm{cv}}, \mathcal{L}_{\mathrm{cv}} & 2.2 .9-2.2 .11 \\
\Delta_{\star} & 2.3 .1 \\
u_{n, v}, \partial_{u_{n, v}} & 2.3 .6-2.3 .8 \\
\mathscr{I}, \mathcal{L}, \overline{\mathrm{As}_{\mathrm{s}}} & 2.4 .6 \\
\varphi_{*}, \varphi^{*}, p_{*}^{d}, i_{d}^{*} & 2.5 .3-2.5 .5 \\
\circledast, \mathrm{MT} & 3.1 .2,3.1 .3
\end{array}
$$




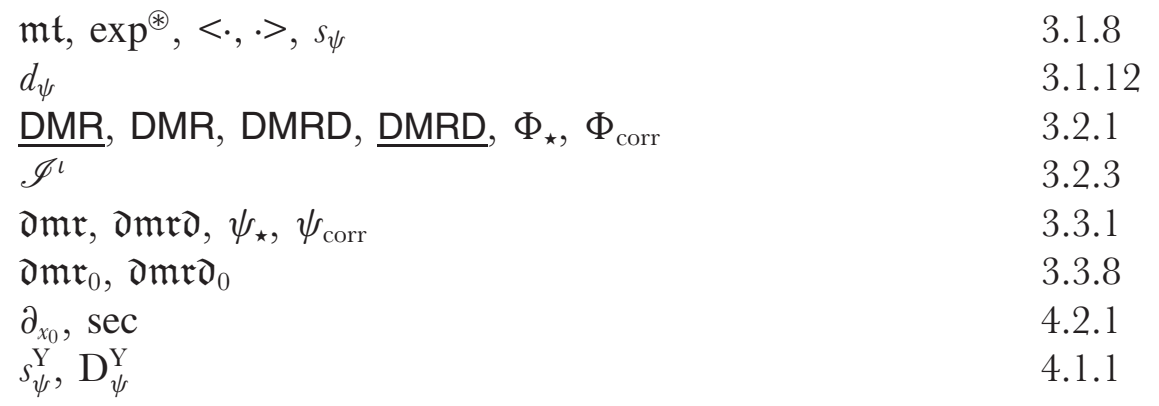

\section{RÉFÉRENCES}

[1] D. Bar-Natan, Non-associative tangles, Geometric topology (Athens, GA, 1993), Amer. Math. Soc., Providence, RI, 1997, pp. 139-183.

[2] D. Bar-Natan, On associators and the Grothendieck-Teichmüller group. I, Selecta Math. (N.S.) 4, No. 2 (1998), 183-212, arXiv:q-alg/960621.

[3] M. Bigotte, G. Jacob, N. Oussous and M. Ретітот, Tables des relations de la fonction zêta colorée, prépublication du LIFL, Université Lille I, 1998.

[4] L. Boutet de Monvel, Remarques sur les séries logarithmiques divergentes, Exposé au colloque «polylogarithmes et conjecture de Deligne-Ihara » au C.I.R.M. (Luminy), http: //www.math.jussieu.fr/ boutet, avril 2000.

[5] D. J. Broadhurst, Conjectured enumeration of irreducible multiple zeta values, from knots and Feynman diagrams, Publication électronique, décembre 1996, arXiv:hep-th/9612012.

[6] P. Cartier, Construction combinatoire des invariants de Vassiliev-Kontsevich des nœuds, R.C.P. 25, Vol. 45 (French) (Strasbourg, 1992-1993), Univ. Louis Pasteur, Strasbourg, 1993, pp. 1-10.

[7] K.-T. CHEN, Integration of paths, geometric invariants and a generalized Baker-Hausdorff formula, Ann. of Math. (2) 65 (1957), 163-178.

[8] K. T. Ghen, Iterated path integrals, Bull. Amer. Math. Soc. 83, no. 5 (1977), 831-879.

[9] P. Deligne, Le groupe fondamental de la droite projective moins trois points, Galois groups over $\mathbf{Q}$ (Berkeley, CA, 1987), Springer, New York, 1989, pp. 79-297.

[10] P. Deligne, Multizeta values, Notes d'exposés, IAS, Princeton, 2001.

[11] P. Deligne, Communication personelle, juin 2001.

[12] M. Demazure and P. Gabriel, Groupes algébriques. Tome I : Géométrie algébrique, généralités, groupes commutatifs, Masson \& Cie, Paris, 1970.

[13] V. G. Drinfel'D, On quasitriangular quasi-Hopf algebras and a group closely related to $\operatorname{Gal}(\overline{\mathbf{Q}} / \mathbf{Q})$, Leningrad Mathematical fournal 2 (1991), 829-860.

[14] J. Écalle, La libre génération des multizêtas et leur décomposition canonico-explicite en irréductibles, Notes de séminaire, automne 1999.

[15] M. Espie, J.-C. Noveldi and G. Racinet, Experimental results about double shuffles of polyzetas, En préparation.

[16] P. Etingof and D. Kazhdan, Quantization of Lie bialgebras. I, Selecta Math. (N.S.) 2, No. 1 (1996), 1-41, arXiv:q-alg/9506005.

[17] I. Gelfand, D. Krob, A. Lascoux, B. Leclerc, V. Retakh and J. Thibon, Non-commutative symmetric functions, Advances in Mathematics 112 (1995), 218-348.

[18] M. Gerstenhaber, The cohomology structure of an associative ring, Ann. of Math. (2) 78 (1963), 267-288.

[19] A. B. Goncharov, Multiple polylogarithms, cyclotomy and modular complexes, Mathematical Research Letters 5 (1998), 497-516.

[20] A. B. Goncharov, The dihedral Lie algebras and Galois symmetries of $\pi_{1}^{(l)}\left(\mathbf{P}^{1}-\left(\{0, \infty\} \cup \mu_{\mathrm{N}}\right)\right)$, Duke Math. J. 110, no. 3 (2001), 397-487, arXiv:math. AG/ 0009121.

[21] A. B. Goncharov, Multiple polylogarithms and mixed Tate motives, Publication électronique, mars 2001, arXiv:math. AG/ 0103059. 
[22] R. Hain and M. Matsumoto, Weighted completion of Galois groups and some conjectures of Deligne, Publication électronique, juin 2000, arXiv:math.AG/0006158.

[23] M. Hoang Ngoc and M. Pетiтot, Lyndon words, polylogarithms and the Riemann zeta function, Discrete Mathematics 217 (1-3) (2000), 273-292.

[24] M. Hoffman, The algebra of multiple harmonic series, fournal of Algebra 194, no. 2 (1997), 477-495.

[25] K. Ihara and M. KaneKo, Derivation relations and regularized double shuffle relations of multiple zeta values, Prépublication, Univ. Kyushu, 2000.

[26] Y. Ihara, Automorphisms of pure sphere braid groups and Galois representations, The Grothendieck Festschrift, Vol. II, Birkhäuser Boston, Boston, MA, 1990, pp. 353-373.

[27] Y. Ihara, Braids, Galois groups, and some arithmetic functions, 1990 Int. Cong. of Mathematicians, Math. Soc. of Japan, Tokyo, 1991, pp. 99-120.

[28] Y. IHARA, On the stable derivation algebra associated with some braid groups, Israel f. Math. 80, no. 1-2 (1992), pp. 135-153,

[29] Y. Ihara and M. Matsumoto, On Galois actions on profinite completions of braid groups, Recent developments in the inverse Galois problem (Seattle, WA, 1993), Amer. Math. Soc., Providence, RI, 1995, pp. 173200.

[30] M. Kontsevitch, Operads and motives in deformation quantization, Lett. Math. Phys. 48, no. 1 (1999), 35-72, Moshé Flato (1937-1998).

[31] T. T. Q. LE and J. Murakami, Kontsevich's integral for the Kauffman polynomial, Nagoya Math. F. 142 (1996), 39-65.

[32] C. Malvenuto and C. Reutenauer, Duality between quasi-symmetric functions and the Solomon descent algebra, Journal of Algebra 177, no. 3 (1995), 967-982.

[33] G. Racinet, Séries génératrices non-commutatives de polyzêtas et associateurs de Drinfel'd, Thèse de doctorat, Université de Picardie-Jules-Verne, 2000, http://www.dma.ens.fr/ racinet.

[34] G. Racinet, Torseurs associés à certaines relations algébriques entre polyzêtas aux racines de l'unité, Comptesrendus de l'Académie des Sciences, Série I 333, no. I (2001), 5-10, arXiv:math.QA/0012024.

[35] G. Racinet, Algèbres de Lie des valeurs formelles d'hyperlogarithmes aux racines de l'unité, Comptes-rendus de l'Académie des Sciences, Série I 333, no. 1 (2001), 11-16, arXiv:math. QA/0012024.

[36] R. REe, Lie elements and an algebra associated with shuffles, Ann. of Math. (2) 68 (1958), 210-220.

[37] G. Reutenauer, Free Lie algebras, London Mathematical Society Monographs, New series, no. 7, Oxford, 1993.

[38] D. E. Tamarkin, Formality of Chain Operad of Small Squares, Publication électronique, septembre 1998, arXiv:math. QA/9809164.

[39] D. Zagier, Values of zeta functions and their applications, First European Congress of Mathematics, Vol. II (Paris, 1992), Birkhäuser, Basel, 1994, pp. 497-512.

\section{G. R.}

Mathematisches Institut, Einsteinstraße 62, D-48149, Münster, racinet@math.uni-muenster.de, http://www.dma.ens.fr/ racinet 